\title{
An Evaluation of Traditional Persian Medicine for the Management of SARS-CoV-2
}

OPEN ACCESS

Edited by:

Michael Heinrich,

UCL School of Pharmacy,

United Kingdom

Reviewed by:

Hesham Rushed El-Seedi, Uppsala University, Sweden Abdolali Mohagheghzadeh, Shiraz University of

Medical Sciences, Iran

*Correspondence: Roja Rahimi

rojarahimi@gmail.com; rahimi_r@tums.ac.ir

Specialty section:

This article was submitted to Ethnopharmacology,

a section of the journal

Frontiers in Pharmacology

Received: 10 June 2020 Accepted: 21 September 2020 Published: 25 November 2020

Citation: Bahramsoltani $R$ and Rahimi R (2020) An Evaluation of Traditional Persian Medicine for the Management of SARS-CoV-2.

Front. Pharmacol. 11:571434. doi: 10.3389/fphar.2020.571434

\author{
Roodabeh Bahramsoltani ${ }^{1,2}$ and Roja Rahimi ${ }^{1,2 *}$ \\ ${ }^{1}$ Department of Traditional Pharmacy, School of Persian Medicine, Tehran University of Medical Sciences, Tehran, Iran, \\ 2 PhytoPharmacology Interest Group (PPIG), Universal Scientific Education and Research Network (USERN), Tehran, Iran
}

A new coronavirus causing severe acute respiratory syndrome (SARS-CoV-2) has emerged and with it, a global investigation of new antiviral treatments and supportive care for organ failure due to this life-threatening viral infection. Traditional Persian Medicine (TPM) is one of the most ancient medical doctrines mostly known with the manuscripts of Avicenna and Rhazes. In this paper, we first introduce a series of medicinal plants that would potentially be beneficial in treating SARS-CoV-2 infection according to TPM textbooks. Then, we review medicinal plants based on the pharmacological studies obtained from electronic databases and discuss their mechanism of action in SARS-CoV2 infection. There are several medicinal plants in TPM with cardiotonic, kidney tonic, and pulmonary tonic activities, protecting the lung, heart, and kidney, the three main vulnerable organs in SARS-CoV-2 infection. Some medicinal plants can prevent "humor infection", a situation described in TPM which has similar features to SARS-CoV-2 infection. Pharmacological evaluations are in line with the therapeutic activities of several plants mentioned in TPM, mostly through antiviral, cytoprotective, anti-inflammatory, antioxidant, and anti-apoptotic mechanisms. Amongst the primarily-introduced medicinal plants from TPM, rhubarb, licorice, garlic, saffron, galangal, and clove are the most studied plants and represent candidates for clinical studies. The antiviral compounds isolated from these plants provide novel molecular structures to design new semisynthetic antiviral agents. Future clinical studies in healthy volunteers as well as patients suffering from pulmonary infections are necessary to confirm the safety and efficacy of these plants as complementary and integrative interventions in SARS-CoV-2 infection.

Keywords: herbal medicine, coronavirus, Traditional Persian medicine, antioxidant, phytochemical

\section{INTRODUCTION}

Coronavirus 2019 (SARS-CoV-2) is a new member of the Coronaviridae family which has caused a global outbreak of a disease called COVID-19 (Wu et al., 2020). Despite the current pharmacotherapies, including different antiviral agents used for the management of hospitalized patients like remdesivir, lotinavir, ritonavir, and ribavirin, a growing number of deaths still occur all over the world (World Health Organization (2020) situation report 197) which has made scientists seek better therapeutic agents. Plants have always been an important source of medicinal 
ingredients, including antiviral agents (Lin et al., 2014; Bahramsoltani et al., 2016). Although there is no approved drug for this disease, it is worth mentioning that oseltamivir (Tamiflu), an antiviral agent currently used in some SARS-CoV2 infected patients, is based on shikimic acid from Illicium verum Hook.f. (star anise) fruit as the precursor (Patra et al., 2020). Additionally, the clinical effectiveness of several medicinal plants such as licorice and garlic has been demonstrated in the treatment of severe acute respiratory syndrome coronavirus (SARS-CoV) and Middle East respiratory syndrome coronavirus (MERS) epidemics (Xiu-hui et al., 2003; Chen and Nakamura, 2004; Cui et al., 2020). A dramatic reduction of mortality from $52 \%$ to $1-4 \%$ was observed during the SARS epidemic in Beijing due to the addition of Traditional Chinese Medicine to conventional therapies (Chen and Nakamura, 2004). Thus, plant-derived natural agents provide a valuable list of compounds with possible antiviral properties against SARSCoV-2 which can be the focus of future investigations. Furthermore, the use of complementary and alternative medicine, including different traditional medicines, can be cost-saving and decrease the prescription of conventional drugs, providing further reasons for scientists to undertake studies on natural products (World Health Organization (2013) WHO traditional medicine strategy: 2014-2023).

Traditional Persian Medicine (TPM) is one of the most ancient medical doctrines mostly known through manuscripts by Persian scientists such as The Canon of Medicine by Avicenna and The Great Continens by Rhazes (Hamedi et al., 2013). Additionally, TPM owes several other scientists with valuable manuscripts regarding anatomy and physiology, disease diagnosis, surgery instruments, and single and compound natural medicines (El-Seedi et al., 2019). TPM has several recommendations for the management of organ damage due to various infections. One of the main approaches in the primary and secondary treatment of diseases in TPM is to protect the four body humors, which are blood, phlegm, bile, and melancholy, from infection (Emtiazy et al., 2012; Kopaei et al., 2016). Many types of infections or the so-called "humor infections" with different clinical manifestations have been described in TPM manuscripts and textbooks, some of which have similar features to that of SARS-CoV-2. One of the pathological conditions explained in TPM for the humors is "humor excitation" which is equivalent to the activation of inflammatory pathways (Aghili Khorasani, 1771).

An examination of pathological conditions similar to COVID-19 along with the therapeutic approaches that are described in TPM manuscripts could pave the way for designing a series of natural products for the management of SARS-CoV-2 infection and related complications (Siahpoosh, 2020). Taking concepts from TPM to treat severe infective pulmonary disorders can help us to select medicinal plants that are potentially useful for SARS-CoV-2 (Kenari et al., 2018). The TPM approach includes lifestyle modifications, along with the administration of some medicinal plants to modify the quality and quantity of the four humors from a pathologic situation into the physiologic condition. The main organs affected in COVID-
19 are the lung, heart, and kidney (Su et al., 2020; Zheng et al., 2020). Accordingly, another approach, which extracts TPM suggestions for the management of the disease is to focus on the natural agents that are specifically recommended as a tonic for these three organs. A "tonic", e.g. a cardiotonic medicine, in TPM is defined as a medicine by which an ideal condition is provided for the physiological functions of an organ so that it is less vulnerable to the pathological conditions (Aghili Khorasani, 1771). This study aims to introduce some of the medicinal plants that TPM claims are effective in the management of symptoms similar to COVID-19 and present current evidence on their efficacy.

\section{METHODS}

This study used several texts, including the Makhzan-al-Adviah, written by MH Aghili Khorasani in 1772 A.D. (Aghili Khorasani, $1771 \mathrm{AD})$, which is the most recent and complete encyclopedia of medicinal materials in TPM, as well as The Great Continens (Rhazes, 960) and The Canon of Medicine (Avicenna, 1025). We searched these books/manuscripts for references to medicinal plants that are described as having protective properties against humor infection and excitation, as well as tonifying and protecting effects on the lungs, heart, and kidney.

Our interpretation and translation of old Persian names into scientific names was based on a book by Ghahreman and Okhovvat (2009), which provides accounts of the most relevant scientific names according to morphological descriptions. The list of plants retrieved during this process was then searched in electronic databases including PubMed, Scopus, Cochrane Library, and Web of Science. Data were collected from inception until March 2020. Only published articles were included in this review and unpublished works were not considered. Language restriction was performed, and English language articles were included. The search terms were the scientific names and common names of each plant combined with "antiviral”, "influenza", "lung", "pulmonary", "alveolar infiltration", "cardiac", "heart”, "cardiomyopathy", "renal”, "kidney", or "immunomodulatory". The inclusion criteria were any in vitro, in vivo, or clinical evidence on the antiviral activity of the selected plants against human pathogenic RNA viruses. Additionally, any study about the beneficial effects of these plants or their isolated phytochemicals on the heart, lungs, and kidneys was included. Studies on animal/plant viral pathogens were excluded. This review does not include in silico antiviral analyses, however, they are referred to in the discussion of the mechanisms and pharmacological effects reported in experiments and studies. The references of the included articles were also searched in order to find additional relevant studies. The plants discussed in the TPM sources and their protective mechanisms are listed in Table $\mathbf{1}$ and the pharmacological evidence obtained from published papers are summarized in Table 2. Table 3 shows a quality assessment of animal studies based on the Animal Research: Reporting of In vivo Experiments (ARRIVE) guidelines (McGrath and Lilley, 2015). 
TABLE 1 | Medicinal plants with possible beneficial effects in treating COVID-19 based on Traditional Persian Medicine.

\begin{tabular}{|c|c|c|c|c|c|c|c|c|}
\hline Scientific name/common name & Persian names & Part & $\begin{array}{l}\text { Preventing } \\
\text { the } \\
\text { infection of } \\
\text { humors }\end{array}$ & $\begin{array}{l}\text { Preventing } \\
\text { the } \\
\text { excitation of } \\
\text { humors }\end{array}$ & $\begin{array}{l}\text { Cardiotonic } \\
\text { properties }\end{array}$ & $\begin{array}{l}\text { Pulmonary tonic } \\
\text { properties }\end{array}$ & $\begin{array}{l}\text { Kidney tonic } \\
\text { properties }\end{array}$ & Reference \\
\hline Acacia nilotica (L.) Delile/Gum Arabic & Samgh-e-Arabi & Gum & & & & + & & (Avicenna 1025; Aghili Khorasani, 1771) \\
\hline Allium sativum L./Garlic & Soom, Sir & Bulb & + & & + & & + & (Aghili Khorasani, 1771) \\
\hline $\begin{array}{l}\text { Alpinia galanga (L.) Willd., } A \text {. } \\
\text { officinarum Hance/Galangal }\end{array}$ & Khoulanjān & Rhizome & & & & + & + & $\begin{array}{l}\text { (Rhazes, 960; Avicenna 1025; Aghili } \\
\text { Khorasani, 1771) }\end{array}$ \\
\hline $\begin{array}{l}\text { Aquilaria malaccensis Lam./Agar } \\
\text { wood }\end{array}$ & Oud-e-Hendi & Wood & & & + & & & (Avicenna 1025; Aghili Khorasani, 1771) \\
\hline Berberis vulgaris L./Barberry & $\begin{array}{l}\text { Zereshk, } \\
\text { ambarbāris (fruit), } \\
\text { Arghis (root) }\end{array}$ & Fruit, root & $\stackrel{+}{+}$ & & $\stackrel{+}{+}$ & & & (Aghili Khorasani, 1771) \\
\hline Cicer arietinum L./Pea & Nokhod & Seed & & & & + & & (Avicenna 1025; Aghili Khorasani, 1771) \\
\hline $\begin{array}{l}\text { Cichorium intybus L./Chicory } \\
\text { Commiphora myrrha (Nees) Engl./ } \\
\text { Myrrh }\end{array}$ & $\begin{array}{l}\text { Kāsni, Hendabā } \\
\text { Morr-e-Macci }\end{array}$ & $\begin{array}{l}\text { Seed, root, leaf } \\
\text { oleo-gum resin }\end{array}$ & $\begin{array}{l}+ \\
+\end{array}$ & + & + & & + & $\begin{array}{l}\text { (Avicenna 1025; Aghili Khorasani, 1771) } \\
\text { (Aghili Khorasani, 1771) }\end{array}$ \\
\hline Coriandrum sativum L./Coriander & $\begin{array}{l}\text { Geshniz, } \\
\text { Kozboreh }\end{array}$ & Fruit & & & + & & & (Rhazes, 960; Aghili Khorasani, 1771) \\
\hline Crocus sativus L./Saffron & Zaferān & Stigma & & & + & + & + & $\begin{array}{l}\text { (Rhazes, 960; Avicenna 1025; Aghili } \\
\text { Khorasani, 1771) }\end{array}$ \\
\hline Cydonia oblonga Mill./Quince & Safarjal, Beh & Fruit & & & + & & & (Aghili Khorasani, 1771) \\
\hline $\begin{array}{l}\text { Cymbopogon schoenanthus (L.) } \\
\text { Spreng./Lemon grass }\end{array}$ & Ezkher & Leaf & & & & + & + & $\begin{array}{l}\text { (Rhazes, 960; Avicenna 1025; Aghili } \\
\text { Khorasani, 1771) }\end{array}$ \\
\hline $\begin{array}{l}\text { Echium amoenum Fisch. \& C.A.Mey./ } \\
\text { Red Feathers }\end{array}$ & $\begin{array}{l}\text { Lesān-al-sour, } \\
\text { gāv zaban }\end{array}$ & Flower & & & & + & & (Avicenna 1025; Aghili Khorasani, 1771) \\
\hline $\begin{array}{l}\text { Elettaria cardamomum (L.) Maton/ } \\
\text { Cardamom }\end{array}$ & $\mathrm{Hel}$ & Seed & & & + & & & (Aghili Khorasani, 1771) \\
\hline Ficus carica L./Fig & Anjeer, Tin & Fruit & & & & + & & (Avicenna 1025; Aghili Khorasani, 1771) \\
\hline $\begin{array}{l}\text { Fumaria parviflora Lam., F. vaillantii } \\
\text { Loisel./Fumitory }\end{array}$ & Shātareh & Aerial parts & & + & & & & (Aghili Khorasani, 1771) \\
\hline Gentiana Iutea L./Yellow Gentian & Gentianā & Root & & & + & & & (Aghili Khorasani, 1771) \\
\hline Glycyrrhiza glabra L./Licorice & $\begin{array}{l}\text { Shirin bayan, } \\
\text { sous }\end{array}$ & Root & & & & + & & $\begin{array}{l}\text { (Rhazes, 960; Avicenna 1025; Aghili } \\
\text { Khorasani, 1771) }\end{array}$ \\
\hline Hordeum vulgare L./Barley & Shaeer, Jo & Seed & & & & + & & $\begin{array}{l}\text { (Rhazes, 960; Avicenna 1025; Aghili } \\
\text { Khorasani, 1771) }\end{array}$ \\
\hline Inula helenium L./Elecampane inula & Rāsan & Root & & & + & + & & (Rhazes, 960; Aghili Khorasani, 1771) \\
\hline Lallemantia royleana (Benth.) Benth. & Bālangu & Seed & & & + & & & (Aghili Khorasani, 1771) \\
\hline Laurus nobilis L./Bay laurel & Ghār, Barg-e-Bou & Leaf, fruit & $\stackrel{+}{+}$ & & & $\stackrel{+}{+}$ & & (Avicenna 1025; Aghili Khorasani, 1771) \\
\hline Malus domestica Borkh./Apple & Sib, Toffāh & Fruit & & & + & & & (Avicenna 1025; Aghili Khorasani, 1771) \\
\hline Melissa officinalis L./Lemon balm & Bādranjbouyeh & Leaf & & & + & & & (Aghili Khorasani, 1771) \\
\hline Nymphaea alba L.Mater lily & Niloufar & Flower & & & & + & & (Avicenna 1025; Aghili Khorasani, 1771) \\
\hline Phyllanthus emblica L./Amla & Ameleh & Fruit & + & & + & & & $\begin{array}{l}\text { (Rhazes, 960; Avicenna 1025; Aghili } \\
\text { Khorasani, 1771) }\end{array}$ \\
\hline Pistacia lentiscus L./Mastic & Mastaki & Oleo-gum-resin & & & & & + & (Aghili Khorasani, 1771) \\
\hline $\begin{array}{l}\text { Plantago major L., P. lanceolata/ } \\
\text { Plantain }\end{array}$ & Bārhang & Seed & & & & & + & (Avicenna 1025; Aghili Khorasani, 1771) \\
\hline Rheum palmatum L./Chinese rhubarb & Reevand & Root & & & & + & & (Aghili Khorasani, 1771) \\
\hline
\end{tabular}




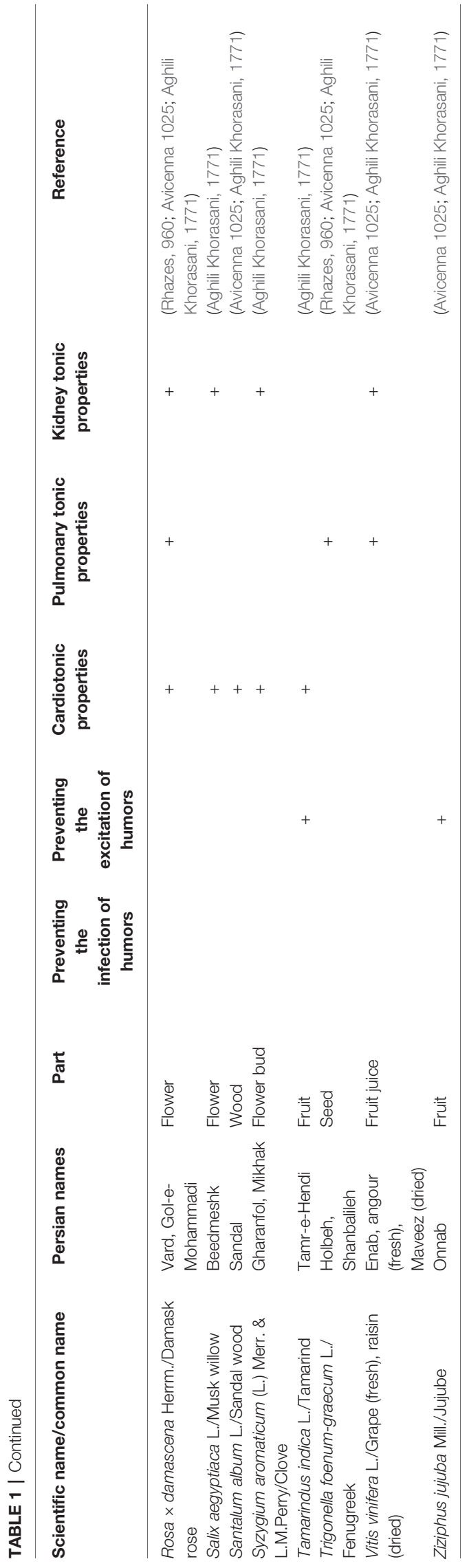

\section{RESULTS}

\section{Amla (Phyllanthus emblica L.)}

Amla is the fruit of a tree from the family Phyllanthaceae, which is used as fresh fruit, jam, or electuary (Yadav et al., 2017). In TPM, amla fruit is considered as a cardiotonic and cardioprotective medicine that is useful for treating cardiovascular problems. Amla also prevents humors from infection and thus acts as a general tonic, i.e. strengthens the body against infection (Aghili Khorasani, $1771 \mathrm{AD})$.

The antiviral activity of amla has been demonstrated against the human immunodeficiency virus (HIV) (Table 2). Polyphenols such as kaempferol and quercetin glycosides, gallotannins, and putranjivain $\mathrm{A}$, a potent non-competitive inhibitor of HIV reverse transcriptase (RT), are known as the main anti-HIV components of amla. The higher inhibitory activity of putranjivain $\mathrm{A}\left(\mathrm{IC}_{50}=3.9 \mu \mathrm{M}\right)$ in comparison to the other isolated phytochemicals $\left(\mathrm{IC}_{50}>200 \mu \mathrm{M}\right)$ from this plant seems to be the result of the hexahydroxydiphenoyl functional group (el-Mekkawy et al., 1995).

In addition to the direct antiviral activity of amla which can suggest natural molecular backbones for possible antiviral agents against SARS-COV-2, there are several reports on the protective effects of the plant on the main organs damaged in SARS-COV-2 infection (Table 2). Pyrogallol, a small gallotanin of amla, demonstrated in vitro protective effect on bronchial epithelial cells of cystic fibrosis infected with $P$. aeruginosa. The compound could significantly prevent bacterial pulmonary inflammation, evident from the reduced production of neutrophil chemokines, pro-inflammatory interleukins (IL), and intercellular adhesion molecule (ICAM)-1, an important contributor in leukocyte chemotaxis (Nicolis et al., 2008).

Additionally, amla could prevent immunotoxicity induced by chromium and arsenic through modulating the phagocytic properties of immune cells, as well as restoring their ability to produce interferon (IFN)- $\gamma$, a critical mediator of the immune system (Sai Ram et al., 2002; Sai Ram et al., 2003).

The cardioprotective effect of amla fruit has been demonstrated in several studies (Table 2). It has also been reported that it prevents the myocardial depletion of creatine kinase-MB (CK-MB), a marker of cardiac damage, and improves hemodynamic parameters, further showing its cardioprotective effects (Patel and Goyal, 2011; Ojha et al., 2012). Emblicanin-A and $\mathrm{B}$, two small-sized hydrolysable gallotanins, have exhibited a protective effect in ischemia-reperfusion (I/R)-induced cardiac damage in rats. Emblicanins could represent cardioprotective properties mostly through the induction of endogenous enzymatic antioxidant defense mechanisms and the prevention of lipid peroxidation. The effect of $50 \mathrm{mg} / \mathrm{kg}$ of this compound was equal to $200 \mathrm{mg} / \mathrm{kg}$ of vitamin $\mathrm{E}$ (standard antioxidant); whereas $100 \mathrm{mg} / \mathrm{kg}$ of emblicanins showed a higher potency compared with vitamin E (Bhattacharya et al., 2002). These compounds have also represented nephroprotective activity in an animal model of cisplatin-induced nephrotoxicity through the same mechanism (Malik et al., 2016). In terms of the cardioprotective effects of amla extract, another target, the phosphoinositide 3-kinase/glycogen synthase kinase $3 \beta$ (PI3K/ 


\begin{tabular}{|c|c|c|c|c|c|}
\hline $\begin{array}{l}\text { General category } \\
\text { of therapeutic } \\
\text { activity }\end{array}$ & $\begin{array}{c}\text { Scientific name/ } \\
\text { preparation }\end{array}$ & Model/Design & $\begin{array}{c}\text { Dosage and } \\
\text { duration of } \\
\text { treatment }\end{array}$ & Mechanisms & Reference \\
\hline
\end{tabular}

Antiviral activity

Allium sativum/diallyl disulfide, diallyl sulfide, alliin

\section{Alpinia galanga/} acetoxychavicol acetate

Alpinia galanga/

acetoxychavicol acetate

\section{Alpinia officinarum \\ diarylheptanoids}

Alpinia officinarum/

diarylheptanoids

Crocus sativus/aqueous

extract, picrocrocin, crocin

Glycyrrhiza glabra/aqueous \& $\quad$ In vitro antiviral activity against HIV-1

alkaline extracts

Glycyrrhiza spp./

glycyrrhizin

Glycyrrhiza spp./

glycyrrhizin

Glycyrrhiza spp./MeOH

In vitro antiviral activity against influenza A $\quad 25-200 \mu \mathrm{g} / \mathrm{m}$ H5N1-infection in human airway epithelia A549 cells

In vitro antiviral activity against clinical -

isolates of SARS coronavirus (FFM-1 \&

$$
\text { FFM-2) }
$$

extract \& isolated compounds

\section{Glycyrrhiza uralensis/} aqueous extract, $18 \beta$ glycyrrhetinic acid, \& glycyrrhizin

In vitro RSV-induced inflammation in human airway epithelial A549 \& HEp-2

Phyllanthus emblica/aqueous In vitro HIV-RT inhibition assay \& $\mathrm{MeOH}$ extract

10-1000 $\mu \mathrm{M}$
\TNF- $\alpha$, IL-8, IL-10, LPO, iNOS

(Hall et al., 2017)

Nuclear export of viral ribonucleoprotein complex $\left(\mathrm{IC}_{50}=12.8 \mu \mathrm{M}\right)$ Ivirus production $\left(\mathrm{IC}_{50}=2 \mu \mathrm{M}, \mathrm{SI}=2.8\right)$

INuclear transport of Rev by direct binding of Cys-529 in chromosomal (Tamura et al., 2009) region maintenance-1 via direct binding to the nuclear export signal of Rev

RSV: $\mathrm{EC}_{50}=5-42 \mu \mathrm{g} / \mathrm{ml}, \mathrm{Sl}=0.9->6.1$

Poliovirus: $E_{50}=3.7-44 \mu \mathrm{g} / \mathrm{ml}, \mathrm{Sl}=1-5.5$

measles virus: $\mathrm{EC}_{50}=6-47 \mu \mathrm{g} / \mathrm{ml}, \mathrm{Sl}=1.3-5.5$

$\downarrow$ Viral messenger RNA \& antigens, No effect on virus adsorption or $\quad$ (Sawamura et al., 2010) invasion,

$\mathrm{EC}_{50}$ of plaque reduction $=16-96 \mu \mathrm{M}$

$\uparrow$ survival,

\Body weight loss \& virus titer is BALF

$\downarrow$ Viral replication by crocin \& picrocrocin:

Crocin:

anti-HIV-1: IC ${ }_{50}: 8 \mu \mathrm{M}, \mathrm{SI}:>187.5$

Picrocrocin:

anti-HIV-1: IC ${ }_{50}: 5 \mu \mathrm{M}$, SI: >600

No significant antiviral effect by the extract

Higher anti-HIV effect with alkaline extract $\left(\mathrm{EC}_{50}=54-167 \mu \mathrm{g} / \mathrm{ml}\right.$,

$\mathrm{Sl}=3-9$ )

Apoptosis, viral replication, IL-6, MCP-1, CCL5, CXCL10, ROS,

$\downarrow N F-K B, J N K, \&$ p38 activation

Viral replication $\left(E C_{50}=300,600 \mathrm{mg} / \mathrm{l}, \mathrm{Sl}=33,67\right)$

Higher activity when added during \& after virus adsorption,

Iviral antigens expression

$I C_{50}(\mu \mathrm{g} / \mathrm{ml})$ :

G. uralensis $\mathrm{MeOH}$ extract: 20, chloroform fraction:8,

glycycoumarin:8.8, liquiritigenin:16.4, licochalcone A:2.5, glycyrin:7.2,

glabridin:6.2, glycyrol:4.6, isoliquiritigenin:3.7, glycyrrhizin:180,

glycyrrhizic acid monoammonium: 320 , Inhibition is mostly in post-entry step

18ß-glycyrrhetinic acid:

IPlaque formation $\left(\mathrm{IC}_{50} \approx 71,75 \mu \mathrm{g} / \mathrm{ml} \& \mathrm{Tl} \approx 71,76\right)$

(Feng Yeh et al., 2013)

Extract:

IPlaque formation (I $\left.\mathrm{C}_{50} \approx 4 \mu \mathrm{g} / \mathrm{ml} \& \mathrm{TI} \approx 27\right)$,

$\downarrow$ viral attachment \& internalization,

$\uparrow I F N \beta$

$\mathrm{C}_{50}=9 \mu \mathrm{g} / \mathrm{ml}$ for aqueous \& $10 \mu \mathrm{g} / \mathrm{ml}$ for $\mathrm{MeOH}$ extracts

putranjivain A from MeOH extract showed $\mathrm{IC}_{50}=3.9 \mu \mathrm{M}$

(el-Mekkawy et al.

1995)

(Esposito et al., 2016) 


\begin{tabular}{|c|c|c|c|c|c|}
\hline $\begin{array}{l}\text { General category } \\
\text { of therapeutic } \\
\text { activity }\end{array}$ & $\begin{array}{l}\text { Scientific name/ } \\
\text { preparation }\end{array}$ & Model/Design & $\begin{array}{l}\text { Dosage and } \\
\text { duration of } \\
\text { treatment }\end{array}$ & Mechanisms & Reference \\
\hline & $\begin{array}{l}\text { Rheum palmatum \& Rheum } \\
\text { officinale/hydromethanolic } \\
\text { extract \& isolated compounds }\end{array}$ & & & $\begin{array}{l}\mathrm{IC}_{50} \text { : } \\
\text { RNase } \mathrm{H} \text { : R. palmatum }=0.9 \mu \mathrm{g} / \mathrm{ml}, R \text {. officinale }=0.25 \mu \mathrm{g} / \mathrm{ml} \text {, sennoside } \\
\mathrm{A}=1.9 \mu \mathrm{M} \text {, sennoside } \mathrm{B}=2.1 \mu \mathrm{M} \\
\text { RDDP: sennoside } \mathrm{A}=5.3 \mu \mathrm{M} \text {, sennoside } \mathrm{B}=2.3 \mu \mathrm{M} \text {, } \\
\text { Integrase: sennoside } \mathrm{A}=3.8 \mu \mathrm{M} \text {, sennoside } \mathrm{B}=87 \mu \mathrm{M} \text {, } \\
\text { Jviral replication }\end{array}$ & \\
\hline & $\begin{array}{l}\text { Rheum palmatum/EtOH } \\
\text { extract }\end{array}$ & $\begin{array}{l}\text { In vitro antiviral activity against } \mathrm{CVB}_{3} \\
\mathrm{CVB}_{3} \text {-induced infection in mouse }\end{array}$ & $\begin{array}{l}\text { In vitro: } 2-10 \mu \mathrm{g} / \\
\mathrm{ml} \\
\text { In vivo: } 0.18-0.5 \\
\mathrm{mg} / \mathrm{kg} / \text { day, i.p., } 5 \\
\text { days }\end{array}$ & $\begin{array}{l}\text { In vitro: Jviral replication }\left(I \mathrm{C}_{50}=4 \mu \mathrm{g} / \mathrm{ml}, \mathrm{Sl}=10\right) \\
\text { In vivo: } \uparrow \text { survival, } \\
\text { Jviral titer }\end{array}$ & (Xiong et al., 2012) \\
\hline & Rheum spp./emodin & In vitro antiviral activity against EV71 & $29.6 \mu \mathrm{M}$ & $\begin{array}{l}\text { WViral replication, maturation, \& virulence, } \\
\text { Lower effect of viral protein expression, } \\
\downarrow \text { cell cycle arrest at S phase }\end{array}$ & (Zhong et al., 2017) \\
\hline & $\begin{array}{l}\text { Rosa damascena/MeOH \& } \\
\text { aqueous extracts, purified } \\
\text { flavonoids }\end{array}$ & In vitro antiviral activity against HIV & - & $\begin{array}{l}\downarrow \text { Infectivity }\left(E C_{50}=4->250 \mu \mathrm{g} / \mathrm{ml}, \mathrm{Sl}=5->100\right) \\
\downarrow \mathrm{gp} 120 \text { binding to } \mathrm{CD} 4, \text { viral protease }\end{array}$ & (Mahmood et al., 1996) \\
\hline & $\begin{array}{l}\text { Syzygium aromaticum/ } \\
\text { aqueous \& MeOH extracts }\end{array}$ & In vitro inhibition of $\mathrm{HCV}$ protease & $100 \mu \mathrm{g} / \mathrm{ml}$ & $\begin{array}{l}76 \% \% 90 \% \text { inhibition by the } \mathrm{MeOH} \& \text { aqueous extracts, } \\
\mathrm{IC}_{50}=33 \mu \mathrm{g} / \mathrm{ml} \text { for aqueous extract }\end{array}$ & (Hussein et al., 2000) \\
\hline & $\begin{array}{l}\text { Syzygium aromaticum/ } \\
\text { eugenol from essential oil }\end{array}$ & $\begin{array}{l}\text { In vitro CPE inhibitory assay against } \\
\text { influenza A virus }\end{array}$ & $5 \mu \mathrm{g} / \mathrm{ml}$ & $\begin{array}{l}\text { \Virus-induced autophagy \& cell death, virus replication, } \\
\downarrow R O S, N O, \text { LPO, IL-1, IL-6, IL-8, TNF- } \alpha \text {, } \\
\text { \activation of ERK1/2, p38MAPK, \& IKK/NF-kB pathways but not } \\
\text { JNK1, } \\
\uparrow G S H, \text { GR, SOD }\end{array}$ & (Dai et al., 2013) \\
\hline & Vitis vinifera/extract & In vitro antiviral activity against HCV & $2.5-20 \mu \mathrm{g} / \mathrm{ml}$ & $\begin{array}{l}\downarrow H C V \text { replication, COX-2, NF-кB \& MAPK/ERK/JNK signaling, } \\
\text { Synergistic effect with conventional anti-HCV drugs }\end{array}$ & (Chen et al., 2016) \\
\hline & $\begin{array}{l}\text { Vitis vinifera/ } \\
\text { proanthocyanidin extract }\end{array}$ & $\begin{array}{l}\text { In vitro RSV-induced inflammation in } \\
\text { human airway epithelial A549 cells }\end{array}$ & $5,10 \mu \mathrm{g} / \mathrm{ml}$ & $\begin{array}{l}\text { \Viral replication, viral nucleoprotein \& fusion protein, } \\
\text { \MUC1, MUC2, MUC5B, MUC8 expression \& mucin synthesis, } \\
\text { Suppression of AP-1 \& NF-KB via p38 MAPKs/JNK }\end{array}$ & (Lee et al., 2017) \\
\hline & $\begin{array}{l}\text { Zizyphus jujuba/betulinic } \\
\text { acid }\end{array}$ & $\begin{array}{l}\text { In vitro antiviral activity against influenza } \\
\text { A/PR/8 virus, } \\
\text { Antiviral activity in mouse infected with } \\
\text { influenza A/PR/8 virus }\end{array}$ & $\begin{array}{l}\text { In vitro: } 0.4-50 \\
\mu \mathrm{g} / \mathrm{ml} \\
\text { In vivo: } 10 \mathrm{mg} / \mathrm{kg} / \\
\text { day, i.p., } 7 \text { days }\end{array}$ & $\begin{array}{l}\text { In vitro: Iviral infection, } \\
\text { In vivo: Ipulmonary necrosis, inflammation, edema, leukocytes } \\
\text { infiltration, } \\
\text { IIFN } \\
\text { No effect on TNF- } \alpha, \text { LL-1 } \beta, \& \text { virus replication }\end{array}$ & (Hong et al., 2015) \\
\hline \multirow[t]{5}{*}{$\begin{array}{l}\text { Cardioprotective } \\
\text { activity }\end{array}$} & Allium sativum/allicin & DOX-induced cardiotoxicity in rat & $\begin{array}{l}20 \mathrm{mg} / \mathrm{kg} / \text { day, } \\
\text { p.o., } 14 \text { days }\end{array}$ & $\begin{array}{l}\uparrow C A T, \text { SOD, Gpx, } \\
\downarrow L P O, L D H, C K-M B, N O, \text { TNF- } \alpha, \text { IL-1 } \beta, \text { COX-2, Casp-3 }\end{array}$ & $\begin{array}{l}\text { (Abdel-Daim et al., } \\
\text { 2017) }\end{array}$ \\
\hline & Allium sativum/alliin & //R-induced cardiotoxicity in mouse & $\begin{array}{l}100 \text { mg/day, i.p., } \\
\text { two doses }\end{array}$ & $\begin{array}{l}\downarrow \text { Cardiomyocyte apoptosis \& infarct size, } \\
\uparrow \text { autophagic flux, LC3II/LC3l, beclin-1, Atg9b }\end{array}$ & (Zhao et al., 2019) \\
\hline & Allium sativum/diallyl trisulfide & $\begin{array}{l}\text { In vitro cytoprotection in H9c2 murine } \\
\text { cardiocyte, } \\
\text { I/R-induced cardiotoxicity in STZ-induced } \\
\text { diabetic rat }\end{array}$ & $\begin{array}{l}\text { In vitro: } 10 \mu \mathrm{M} \text {, } \\
\text { In vivo: } 20 \mathrm{mg} / \mathrm{kg} / \\
\text { day, p.o., } 3 \text { days }\end{array}$ & $\begin{array}{l}\text { In vitro \& in vivo: } \\
\downarrow \text { Apoptosis, } \\
\uparrow A M P K-\text { mediated AKT/GSK-3ß/HIF- } 1 \alpha \text { activation }\end{array}$ & (Yu et al., 2017) \\
\hline & Allium sativum/homogenate & $\begin{array}{l}\text { Fructose-induced cardiotoxicity in } \\
\text { diabetic rat }\end{array}$ & $\begin{array}{l}250 \mathrm{mg} / \mathrm{kg} / \mathrm{day} \text {, } \\
\text { p.o., } 8 \text { weeks }\end{array}$ & $\begin{array}{l}\downarrow N F-k B, R O S, L P O, N O \\
\uparrow C A T, G S H, G p x, S O D, \\
\text { Modulation of PI3K/AKT/Nrf2-Keap1 pathway }\end{array}$ & (Padiya et al., 2014) \\
\hline & Alpinia galanga/Cardamonin & DOX-induced cardiotoxicity in mouse & & & (Qi et al., 2020) \\
\hline
\end{tabular}




\begin{tabular}{|c|c|c|c|c|c|}
\hline $\begin{array}{l}\text { General category } \\
\text { of therapeutic } \\
\text { activity }\end{array}$ & $\begin{array}{l}\text { Scientific name/ } \\
\text { preparation }\end{array}$ & Model/Design & $\begin{array}{l}\text { Dosage and } \\
\text { duration of } \\
\text { treatment }\end{array}$ & Mechanisms & Reference \\
\hline & & & $\begin{array}{l}20-80 \mathrm{mg} / \mathrm{kg} / \text { day, } \\
\text { p.o., } 28 \text { days }\end{array}$ & $\begin{array}{l}\text { Improvement of cardiac function, } \\
\uparrow N r f-2 \text { signaling, HO-1, NQO-1, GCLM, SOD, GSH, CAT, } \\
\downarrow L P O, \text { ROS, \& apoptosis }\end{array}$ & \\
\hline & $\begin{array}{l}\text { Crocus sativus/aqueous } \\
\text { extract }\end{array}$ & ISO-induced cardiotoxicity in rat & $\begin{array}{l}200-800 \mathrm{mg} / \mathrm{kg} / \\
\text { day, p.o., } 28 \text { days }\end{array}$ & $\begin{array}{l}\text { Improvement of hemodynamic function of heart, } \\
\uparrow \text { Cardiac SOD, CAT, GSH, } \\
\downarrow L P O, \text { LDH \& CK-MB leakage }\end{array}$ & (Sachdeva et al., 2012) \\
\hline & $\begin{array}{l}\text { Crocus sativus/aqueous } \\
\text { extract, safranal }\end{array}$ & ISO-induced cardiotoxicity in rat & $\begin{array}{l}20-160 \mathrm{mg} / \mathrm{kg} / \\
\text { day of extract or } \\
0.025-0.075 \text { of } \\
\text { safranal, i.p., } 9 \\
\text { days }\end{array}$ & $\begin{array}{l}\downarrow \mathrm{LDH}, \mathrm{CK}-\mathrm{MB}, \mathrm{LPO}, \\
\text { Improvement of myocardium morphological changes }\end{array}$ & $\begin{array}{l}\text { (Mehdizadeh et al., } \\
\text { 2013) }\end{array}$ \\
\hline & Crocus sativus/crocin & $\begin{array}{l}\text { In vitro LPS-induced cardiotoxicity in } \\
\text { H9c2 murine cardiocyte }\end{array}$ & $10-40 \mu \mathrm{M}$ & $\begin{array}{l}\uparrow \text { Viability, thiol content } \\
\downarrow \text { TNF- } \alpha, \text { PGE2, IL-1 } 1 \beta \text {, and IL-6, NO, } \\
\downarrow \text { TNF- } \alpha, \text { COX-2, IL-1 } 1 \beta, \text { IL-6, \& iNOS gene expression }\end{array}$ & (Rahim et al., 2019) \\
\hline & Crocus sativus/crocin & DOX-induced cardiotoxicity in rat & $\begin{array}{l}20,40 \mathrm{mg} / \mathrm{kg} / \text { day, } \\
\text { i.p., } 20 \text { days }\end{array}$ & Improvement of heart function, ECG, \& histopathological damages & (Razmaraii et al., 2016) \\
\hline & $\begin{array}{l}\text { Crocus sativus/ } \\
\text { hydromethanolic extract }\end{array}$ & $\begin{array}{l}\text { In vitro } 1 / \mathrm{R}+\mathrm{DOX} \text {-induced toxicity in } \mathrm{H} 9 \mathrm{c} 2 \\
\text { murine cardiocyte }\end{array}$ & $10 \mu \mathrm{g} / \mathrm{ml}$ & $\begin{array}{l}\uparrow \text { Viability, } \alpha \text {-actinine, troponine C \& MLC, AKT/P70S6K \& ERK1/2 } \\
\text { activity, } \\
\downarrow \text { Casp-3, LDH, mitochondrial dysfunction }\end{array}$ & (Chahine et al., 2016) \\
\hline & $\begin{array}{l}\text { Glycyrrhiza glabra/aqueous } \\
\text { extract }\end{array}$ & $\begin{array}{l}\text { In vitro DOX-induced toxicity in } \mathrm{H} 9 \mathrm{c} 2 \\
\text { murine cardiocyte }\end{array}$ & $20-200 \mu \mathrm{g} / \mathrm{ml}$ & 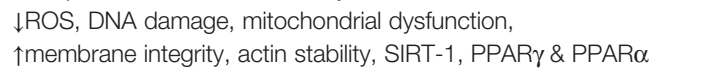 & (Upadhyay et al., 2020) \\
\hline & $\begin{array}{l}\text { Glycyrrhiza glabra/ } \\
\text { hydroalcoholic extract }\end{array}$ & //R-induced cardiotoxicity in rat & $\begin{array}{l}400 \mathrm{mg} / \mathrm{kg}, \text { p.o., } \\
30 \text { days }\end{array}$ & $\begin{array}{l}\uparrow S O D, G p x, C A T, G S H, \\
\text { Restoration of LDH, CK-MB, \& hemodynamic cardiac function, } \\
\downarrow L P O\end{array}$ & (Ojha et al., 2013) \\
\hline & $\begin{array}{l}\text { Glycyrrhiza spp./glycyrrhizic } \\
\text { acid }\end{array}$ & ISO-induced cardiotoxicity in rat & $\begin{array}{l}10,20 \mathrm{mg} / \mathrm{kg}, \\
\text { p.o., } 2 \text { days }\end{array}$ & $\begin{array}{l}\text { Modulation of ECG \& morphology, } \\
\downarrow C K-M B, L D H \\
\text { Reversible inhibition of L-type } \mathrm{Ca}^{2+} \text { channels in isolated rat } \\
\text { cardiomyocytes ( } \mathrm{EC}_{50}=145.54 \mu \mathrm{g} / \mathrm{mL} \text { ) }\end{array}$ & (Li et al., 2019) \\
\hline & $\begin{array}{l}\text { Phyllanthus emblica/aqueous } \\
\text { extract }\end{array}$ & I/R-induced cardiotoxicity in rat & $\begin{array}{l}100 \mathrm{mg} / \mathrm{kg} / \text { day, } \\
\text { p.o., } 30 \text { days }\end{array}$ & 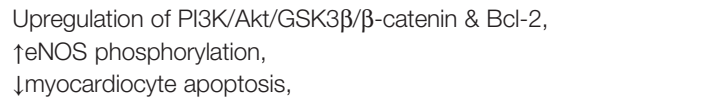 & $\begin{array}{l}\text { (Thirunavukkarasu et al. } \\
\text { 2015) }\end{array}$ \\
\hline & $\begin{array}{l}\text { Phyllanthus emblica/ } \\
\text { emblicanin-A \& -B enriched } \\
\text { fraction }\end{array}$ & I/R-induced cardiotoxicity in rat & $\begin{array}{l}100 \& 200 \mathrm{mg} / \mathrm{kg} / \\
\text { day, p.o., } 14 \text { days }\end{array}$ & $\begin{array}{l}\uparrow \text { Cardiac SOD, CAT, Gpx, } \\
\downarrow L P O\end{array}$ & $\begin{array}{l}\text { (Bhattacharya et al., } \\
\text { 2002) }\end{array}$ \\
\hline & $\begin{array}{l}\text { Phyllanthus emblical } \\
\text { hydroalcoholic extract }\end{array}$ & ISO-induced cardiotoxicity in rat & $\begin{array}{l}100-500 \mathrm{mg} / \mathrm{kg} / \\
\text { day, p.o., } 30 \text { days }\end{array}$ & $\begin{array}{l}\text { Restoration of hemodynamic parameters \& cardiac function, } \\
\uparrow \text { Cardiac SOD, CAT, Gpx, GSH, } \\
\downarrow \text { LPO, } \\
\downarrow \text { LDH \& CK-MB leakage from myocardium }\end{array}$ & (Ojha et al., 2012) \\
\hline & Phyllanthus emblica/juice & $\begin{array}{l}\text { STZ-induced diabetic myocardial } \\
\text { dysfunction in rat }\end{array}$ & $\begin{array}{l}1 \mathrm{ml} / \mathrm{kg} / \text { day, p.o., } \\
8 \text { weeks }\end{array}$ & $\begin{array}{l}\text { Restoration of hemodynamic parameters, } \\
\downarrow \downarrow L D H \& \text { CK-MB }\end{array}$ & (Patel and Goyal, 2011) \\
\hline & Rheum officinale/emodin & Isolated perfused beating rabbit atria & $10-100 \mu \mathrm{M}$ & $\begin{array}{l}\uparrow \text { Atrial natriuretic peptide, } \\
\text { \atrial pulse pressure \& stroke volume, } \\
\text { Involvement of L-type } \mathrm{Ca}^{2+} \& \mathrm{~K}^{+} \text {channel, } \\
\text { No change in muscarinic system }\end{array}$ & (Zhou et al., 2014) \\
\hline & $\begin{array}{l}\text { Rheum palmatum/ } \\
\text { chrysophanol }\end{array}$ & & $\begin{array}{l}\text { In vitro: } 1-20 \mu \mathrm{M} \\
\text { In vivo: } 5-40 \mathrm{mg} /\end{array}$ & $\begin{array}{l}\text { In vitro: Japoptosis, cleavage \& activation of PARP1, Casp-3, } \\
\text { cytochrome c release from mitochondria to cytoplasm, }\end{array}$ & (Lu et al., 2019) \\
\hline
\end{tabular}




\section{General category}

Scientific name/

Model/Design

Dosage and

duration of

treatment

activity

preparation

Reference

(n)

In vitro DOX-induced toxicity in $\mathrm{H} 9 \mathrm{c} 2$ murine cardiocyte,

DOX-induced cardiotoxicity in rat

Rheum palmatum/rhein

Rosa damascena/

hydroethanolic extract

Trigonella foenum-graecum/ digoxigenin-3-O-rutin

Trigonella foenum-graecum polysaccharide

\section{cardiocyte}

Isolated guinea pig heart un-treated or $\quad 0.1-1 \mathrm{mg} \%$

pretreated with propranolol

methacholine, \& diltiazem

ISO-induced cardiotoxicity in rat

In vitro cytoprotection in $\mathrm{H} 9 \mathrm{c} 2$ murine cardiocyte,

Thiamethoxam-induced cardiotoxicity in

rat
Trigonella foenum-graecum powder

Trigonella foenum-graecum seed powder

Trigonella foenum-graecum seed powder

trigonelline

ISO+ hypercholesterolemic diet-induced cardiotoxicity in rat

STZ-induced cardiotoxicity in diabetic

rats

STZ-induced cardiotoxicity in diabetic rats

ISO-induced cardiotoxicity in rat

Vitis vinifera/proanthocyanidin DOX-induced cardiotoxicity in rat extract

Vitis vinifera/proanthocyanidin In vitro I/R-induced cardiotoxicity in H9c2 $50-200 \mu \mathrm{g} / \mathrm{m}$ extract murine cardiocyte

Zizyphus jujuba/Jujuboside A murine cardiocytes

Zizyphus jujuba/polyphenols ISO-induced cardiotoxicity in rat

Immunomodulatory Allium sativum/aged extract activity

Allium sativum/essential oil \&

Randomized, double-blind, placebocontrolled trial in healthy individuals organosulfur compounds

Allium sativum/

polysaccharides macrophages
$5-200 \mu \mathrm{g} / \mathrm{ml}$
ISO-induced cardiotoxicity in $\mathrm{H9c2}$ kg/day, p.o., 7 days

$1 \mu \mathrm{g} / \mathrm{m}$

$\uparrow \mathrm{Bcl}-2 / \mathrm{Bax}$,

In vivo: modulation of ECG

ffibrosis, apoptosis, mitochondrial damage

Apoptosis, ROS, p-P38,

$\uparrow A K T$ \& GSK3ß phosphorylation

$\uparrow$ Heart rate \& contractility,

Higher activity of the extract vs. isoprenaline

et al., 2018)

(Boskabady et al.

2013)

$2.5-10 \mathrm{mg} / \mathrm{kg} / \quad \downarrow C K-M B, C r$, LDH, AST, ALT, LPO,

day, p.o., 10 days $\uparrow G S H$, Gpx, GST, SOD, CAT, $\mathrm{Na}^{+}-\mathrm{K}^{+}$-ATPase

0.01-1 $\mathrm{mg} / \mathrm{ml}$, In vitro: $\downarrow$ H9c2 necrosis \& apoptosis,

$100 \& 200$ mg/kg/ In vivo: $\downarrow L D H, C P K$, AST, troponin-T, LDL, TAG, LPO \& protein

(Panda and Kar, 2010)

(Feki et al., 2019)

day, p.o., 30 days oxidation,

$10 \%$ of diet, p.o., $\quad \downarrow L D H, C K-M B, A S T, A L T$,

8 weeks Improvement of the lipid profile \& histology of the heart muscle

$9 \mathrm{~g} / \mathrm{kg} /$ day, p.o., $\quad \uparrow$ Activity of cardiac SOD, CAT, GSH, GST

30 days

IRAS activity, type IV collagen, fibronectin, Bax, 4-hydroxynonenal,

weight, 6 weeks iNOS, nitrate/nitrite

$\uparrow$ PUFA/SFA ratio

20-80 mg/kg/day, \Infarction area

p.o., 20 days $\quad \downarrow C K-M B, L D H$, LPO, ALT,

$\downarrow$ Hsp27 \& $\alpha$ B crystallin,

$\uparrow$ GSH, Gpx, GST, SOD, CAT

$70 \mathrm{mg} / \mathrm{kg}$, p.o., Modulation of ECG,

10 days $\downarrow$ CK-MB, LDH, LPO,

$\uparrow S O D, C A T$

$\uparrow$ Viability,

ILDH, GRP78, CHOP, phosphorylated PERK, elF2 $\alpha$, endoplasmic

reticulum stress-induced apoptosis

5-20 $\mu \mathrm{M} \quad \uparrow$ Viability, phosphorylation of PI3K, Akt, \& mTOR,

$\downarrow L C 3-1 / / 1$

$300 \mathrm{mg} / \mathrm{kg} / \mathrm{day}, \quad \downarrow L \mathrm{LPO}, \mathrm{Ca}^{2+} \& \mathrm{Mg}^{2+}$-ATPase activity,

p.o., 5,10 days $\uparrow S O D, G p x$,

$\mathrm{Na}^{+} \mathrm{K}^{+}$-ATPase, LDH \& CK activity,

Modulation of ECG

$2.6 \mathrm{~g} /$ day, p.o., $\quad \uparrow$ Proliferation of $\gamma \delta-T$ cells \& NK cells,

90 days $\quad \downarrow$ severity of cold \& flu symptoms

$\uparrow \mathrm{Ca}^{2+}$ flux $\left(\mathrm{EC}_{50}=9-22 \mu \mathrm{M}\right)$,

$\uparrow R O S$ production via PI3K activation,

CREB, ERK1/2, \& GSK-3 $\alpha / \beta$ phosphorylation (200-500 $\mu \mathrm{M}$ of 1,3-

dithiane)

Prolifer

Higher activity by the fresh sample

$0.3-10 \mu \mathrm{M}$

JIFN $\beta$ mRNA expression, NF-KB activation, NO production via TLR-3
(Mukthamba and

Srinivasan, 2015)

(Tripathi and Chandra,

2009)

(Pradeep and

Srinivasan, 2018)

(Panda et al., 2013)

(Ammar el et al., 2013)

(Wang X. et al., 2017)

(Han et al., 2016)

(Cheng et al., 2012)

(Nantz et al., 2012)

(Schepetkin et al., 2019)

(Li et al., 2017)

(Ando et al., 2005) 


\begin{tabular}{|c|c|c|c|c|c|}
\hline $\begin{array}{l}\text { General category } \\
\text { of therapeutic } \\
\text { activity }\end{array}$ & $\begin{array}{l}\text { Scientific name/ } \\
\text { preparation }\end{array}$ & Model/Design & $\begin{array}{l}\text { Dosage and } \\
\text { duration of } \\
\text { treatment }\end{array}$ & Mechanisms & Reference \\
\hline & $\begin{array}{l}\text { Alpinia galanga/ } \\
\text { acetoxychavicol acetate }\end{array}$ & $\begin{array}{l}\text { In vitro LPS-activated murine } \\
\text { macrophage }\end{array}$ & & & \\
\hline & Alpinia galanga/galangin & $\begin{array}{l}\text { In vitro LPS-activated RAW } 264.7 \\
\text { macrophages }\end{array}$ & 12.5- $50 \mu \mathrm{M}$ & 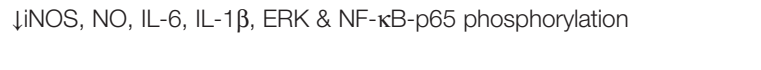 & (Jung et al., 2014) \\
\hline & $\begin{array}{l}\text { Cichorium intybus/EtOH } \\
\text { extract }\end{array}$ & EtOH-induced immunotoxicity in mouse & $\begin{array}{l}300 \mathrm{mg} / \mathrm{kg} / \text { day, } \\
\text { p.o., } 28 \text { days }\end{array}$ & $\begin{array}{l}\uparrow \text { Circulating leukocytes, splenic plaque forming cells, hemagglutination } \\
\text { titers to SRBC, secondary IgG response to bovine serum albumin, } \\
\text { phagocytes activity, NK cells, IFN } \gamma \text {, delayed-type hypersensitivity }\end{array}$ & (Kim et al., 2002) \\
\hline & Cichorium intybus/powder & $\begin{array}{l}\text { Innate immune response in growing } \\
\text { piglets }\end{array}$ & $\begin{array}{l}4 \% \text { as dietary } \\
\text { supplement, p.o., } \\
21 \text { days }\end{array}$ & $\begin{array}{l}\downarrow \text { Apolipoprotein C-II complement component C6, CRP, CD14 antigen, } \\
\text { C4b binding protein } \alpha \& \beta \text { chains, fibrinogen }\end{array}$ & (Lepczynski et al., 2015) \\
\hline & $\begin{array}{l}\text { Crocus sativus/hydroethanolic } \\
\text { extract }\end{array}$ & $\begin{array}{l}\text { Non-stimulated \& phytohemagglutinin- } \\
\text { stimulated lymphocytes }\end{array}$ & $50-500 \mu \mathrm{g} / \mathrm{ml}$ & $\begin{array}{l}\text { Stimulated cells: } \downarrow \text { IFN } \gamma, \mathrm{IL}-10 \text {, } \\
\text { Non-stimulated cells: } \uparrow \mathrm{IFN} \gamma, \mathrm{LL}-4 \\
\uparrow T h 1 / T h 2 \text { balance }\end{array}$ & $\begin{array}{l}\text { (Boskabady et al., } \\
\text { 2011) }\end{array}$ \\
\hline & Crocus sativus/powder & $\begin{array}{l}\text { Randomized double-blind placebo- } \\
\text { controlled in healthy men }\end{array}$ & $\begin{array}{l}100 \mathrm{mg}, \text { p.o., } 6 \\
\text { weeks }\end{array}$ & $\begin{array}{l}\text { Week 3: } \uparrow \text { lgG, monocyte percentage, } \\
\downarrow \text { lgM, basophil percentage, } \\
\text { Week 6: all results returned to baseline }\end{array}$ & $\begin{array}{l}\text { (Kianbakht and Ghazavi, } \\
\text { 2011) }\end{array}$ \\
\hline & Glycyrrhiza spp./glycyrrhizin & $\begin{array}{l}\text { In vitro LPS-induced inflammation in } \\
\text { RAW } 264.7 \text { macrophage, } \\
\text { LPS-induced endotoxemia in mouse }\end{array}$ & $\begin{array}{l}\text { In vitro: } 0.5-2 \mu \mathrm{M} \text {, } \\
\text { In vivo: } 200 \mathrm{mg} / \\
\mathrm{kg}, \text { i.p., single } \\
\text { dose }\end{array}$ & $\begin{array}{l}\uparrow H O-1 \text {, nuclear translocation of Nrf2, } \\
\downarrow H M G B 1 \text {, iNOS, } \\
\text { Involvement of p38, but not ERK or JNK }\end{array}$ & (Kim et al., 2015) \\
\hline & $\begin{array}{l}\text { Phyllanthus emblica/EtOH } \\
\text { extract }\end{array}$ & $\begin{array}{l}\text { In vitro Chromium (VI)-induced } \\
\text { immunosuppression in rat lymphocyte }\end{array}$ & $10-1000 \mu \mathrm{g} / \mathrm{ml}$ & $\begin{array}{l}\text { Restoration of IL-2 \& IFN } \gamma \text { production } \\
\uparrow c e l l \text { survival, GSH, Gpx, } \\
\downarrow L D H \text { leakage, ROS, LPO, DNA fragmentation }\end{array}$ & (Sai Ram et al., 2002) \\
\hline & $\begin{array}{l}\text { Phyllanthus emblical } \\
\text { hydroalcoholic extract }\end{array}$ & $\begin{array}{l}\text { In vitro Chromium (VI)-induced } \\
\text { immunosuppression in J-774 } \\
\text { macrophage }\end{array}$ & $250 \mu \mathrm{g} / \mathrm{ml}$ & $\begin{array}{l}\text { Restoration of phagocytosis \& IFN } \gamma \text { production, } \\
\text { †cell survival, Gpx \& GSH, } \\
\downarrow \text { ROS }\end{array}$ & (Sai Ram et al., 2003) \\
\hline & Syzygium aromaticum/biflorin & $\begin{array}{l}\text { In vitro LPS-induced inflammation in } \\
\text { RAW } 264.7 \text { macrophage, } \\
\text { LPS-induced endotoxemia in mouse }\end{array}$ & $\begin{array}{l}5 \& 10 \mathrm{mg} / \mathrm{kg} \\
\text { i.p., single dose }\end{array}$ & liNOS, NO, COX-2, PG-E2, TNF- $\alpha$, IL-6, p-STAT1, p-p38 & (Lee et al., 2016) \\
\hline & $\begin{array}{l}\text { Syzygium aromaticum/ } \\
\text { essential oil }\end{array}$ & $\begin{array}{l}\text { Cyclophosphamide-induced } \\
\text { immunosuppression in mouse under } \\
\text { SRBC challenge }\end{array}$ & $\begin{array}{l}100-400 \mathrm{mg} / \mathrm{kg} / \\
\text { day, p.o., } 7 \text { days }\end{array}$ & $\begin{array}{l}\uparrow W B C \text {, } \\
\text { Stimulation of both humoral \& cell-mediated immunity }\end{array}$ & (Carrasco et al., 2009) \\
\hline & $\begin{array}{l}\text { Syzygium aromaticum/ } \\
\text { hydromethanolic extract \& } \\
\text { eugenol }\end{array}$ & $\begin{array}{l}\text { In vitro LPS-induced inflammation in } \\
\text { murine peritoneal macrophage }\end{array}$ & $5-100 \mu \mathrm{g} / \mathrm{well}$ & $\begin{array}{l}\downarrow \| L-1 \beta \text { by the extract, } \\
\downarrow \| L-6 \text { \& IL- } 10 \text { by the extract \& eugenol }\end{array}$ & (Bachiega et al., 2012) \\
\hline & $\begin{array}{l}\text { Trigonella foenum-graecum/ } \\
\text { powder }\end{array}$ & $\begin{array}{l}\text { Burn wound induced in } \\
\text { cyclophosphamide-immunosuppressed } \\
\text { rat }\end{array}$ & $\begin{array}{l}0.5 \& 1 \mathrm{~g} / \mathrm{kg} / \text { day, } \\
\text { p.o., } 28 \text { days }\end{array}$ & $\begin{array}{l}\downarrow \text { Neutropenia \& lymphopenia, } \\
\uparrow \text { weight \& cellularity of thymus, spleen, bone marrow, } \\
\uparrow \gamma \text {-globulin, delayed-type hypersensitivity response, \& burn wound } \\
\text { healing rate }\end{array}$ & (Ramadan et al., 2011) \\
\hline & $\begin{array}{l}\text { Vitis vinifera/proanthocyanidin } \\
\text { extract }\end{array}$ & $\begin{array}{l}\text { Aflatoxin-induced immunotoxicity in } \\
\text { mouse }\end{array}$ & $\begin{array}{l}50,100 \mathrm{mg} / \mathrm{kg} / \\
\text { day, p.o., } 3 \text { weeks }\end{array}$ & 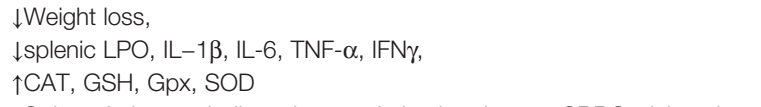 & (Long et al., 2016) \\
\hline & $\begin{array}{l}\text { Zizyphus jujuba cv. Huizao/ } \\
\text { acidic polysaccharides }\end{array}$ & $\begin{array}{l}\text { Immunomodulatory effect in healthy } \\
\text { mouse }\end{array}$ & $\begin{array}{l}50-200 \mathrm{mg} / \mathrm{kg} / \\
\text { day, p.o., } 7 \text { days }\end{array}$ & $\begin{array}{l}\uparrow \text { Spleen \& thymus indices, hemagglutination titers to SRBC, delayed- } \\
\text { type hypersensitivity, phagocytes activity }\end{array}$ & (Zou et al., 2018) \\
\hline $\begin{array}{l}\text { Lung protective } \\
\text { activity }\end{array}$ & $\begin{array}{l}\text { Allium sativum/aqueous } \\
\text { extract }\end{array}$ & $\begin{array}{l}\text { Lambda-cyhalothrin-induced pulmonary } \\
\text { damage in rat }\end{array}$ & $\begin{array}{l}100 \mathrm{mg} / \mathrm{kg} / \mathrm{day} \text {, } \\
\text { i.p., } 21 \text { days }\end{array}$ & $\downarrow$ Cough, nasal discharge, alveolitis, lung inflammation \& hyperplasia & $\begin{array}{l}\text { (Mohi El-Din et al., } \\
\text { 2014) }\end{array}$ \\
\hline
\end{tabular}




\section{General category}

Scientific name/

Model/Design

Dosage and

duration of

Mechanisms

Reference

activity

treatment

Allium sativum/S-allyl-L-

Bleomycin-induced pulmonary toxicity in $\quad 5,10 \mathrm{mg} / \mathrm{kg}$, i.p.,

cysteine

single dose

IPulmonary fibrosis via $\alpha$-SMA, TNF- $\alpha$, fibronectin, collagen I \& III,

leukocytes infiltration into BALF, iNOS, AKT \& NF-kB p65

phosphorylation

Allium sativum/S-

allylmercaptocysteine

LPS-induced lung inflammation in mouse $\quad 10-60 \mathrm{mg} / \mathrm{kg}$,

Alpinia galanga/ p.o., single dose

$\downarrow$ Lung edema, MPO, TNF- $\alpha$, IL-1 $\beta$, IL-6, iNOS, COX-2, NF-kB, LPO,

(Nie et al., 2019)

acetoxychavicol acetate

OVA-induced airway inflammation in

$25,50 \mathrm{mg} / \mathrm{kg} / \mathrm{day}$

GSH, SOD, HO-1,

Modulation of Keap1/Nrf2 pathways

Alpinia galanga/galangin LPS-induced airway inflammation in

i.p., 5 days

cells hyperplasia,

Lanti-OVA IgG, IL-4, IL-13, IL-12 $\alpha$, IFN $\gamma$

$1.5,15 \mathrm{mg} / \mathrm{kg} / \quad \uparrow \mathrm{HO}-1$ \& oxygenation

day, i.p., single Ilung edema, NF-KB, MPO, IL-6, TNF- $\alpha$ mouse

$10 \mu \mathrm{M}$ in vitro

In vitro: \MCP-1, nuclear translocation of p65, eotaxin, CXCL10, and

(Mo et al., 2020)

Alpinia galanga/galangin

In vitro TNF- $\alpha$-induced inflammation in normal human airway smooth muscle

$5,15 \mathrm{mg} / \mathrm{kg} /$ day, $\mathrm{VCAM}-1$,

cells, $\quad$ i.p., 3 days

VCAM-1,

OVA-induced airway inflammation in

In vivo: leukocyte infiltration in BALF, airway hyperresponsiveness,

goblet cells hyperplasia,

Lanti-OVA IgG, IL-4, IL-5, IL-13, iNOS, VCAM-1, NF-kB-related

inflammation

Crocus sativus/crocin

In vitro cytoprotection in HUVEC cells,

In vitro: $20 \mu \mathrm{M}$,

In vitro \& in vivo: $\downarrow$ NF- $\mathrm{KB}$ \& MAPK activity, MMP-9, heparanase

LPS-induced lung inflammation in mouse In vivo: $15-60 \mathrm{mg} / \uparrow$ Pulmonary vascular permeability

kg, i.p., 7 days

Crocus sativus/crocin

Bleomycin-induced pulmonary toxicity in

$\downarrow$ Pulmonary inflammation, fibrosis, leukocytes infiltration into BALF

$\downarrow L D H$, LPO, NO, TNF- $\alpha$, TGF- $\beta 1$, TLR-4, IL-10,

$\uparrow S O D, G S H, T A C, H O-1$, Nrf2

Crocus sativus/powder Randomized, triple-blind, placebo-

controlled trial in patients with mild \&

moderate persistent asthma

Crocus sativus/safranal

OVA-induced airway inflammation in

guinea pigs

Glycyrrhiza glabra/glycyrrhizic OVA-induced airway inflammation in mouse

$100 \mathrm{mg} /$ day, p.o., Improvement of spirometry parameters,

8 weeks

\CRP, anti-Hsp70 antibody

\section{$4-16 \mu \mathrm{g} / \mathrm{ml}$ of \\ INO, nitrite, IL-4, tracheal response to methacholine \& OVA,}

drinking water

$10-40 \mathrm{mg} / \mathrm{kg} / \mathrm{day}$

$\uparrow I F N \gamma / \mathrm{LL}-4$

acid

p.o., 30 days

$\uparrow$ Regulatory T cells, IFN $\gamma$, Foxp3 protein,

Glycyrrhiza glabra/glycyrrhizin In vitro TGF- $\alpha$-induced mucus production

in $\mathrm{NCl}-\mathrm{H} 292$ cells,

$\mu \mathrm{M}$,

PS \& IL-4-induced airway inflammation In vivo: 15-135 in mouse

In vivo: $15-135$

$\mathrm{mg} / \mathrm{kg}$, s.c., 6

days

Glycyrrhiza glabra/aqueous

extract

Randomized, double-blind, placebo-

$0.5 \mathrm{~g} / 30 \mathrm{ml}$, as

$\downarrow$ leukocytes infiltration into BALF, IL-4, IL-5, IL-13, OVA-specific IgE

In vitro: $\downarrow$ MUC5AC protein and mRNA expression

In vivo: $\downarrow$ Goblet cell hyperplasia \& MUC5AC mRNA expression

(Seo et al., 2013)

(Shu et al., 2014)

(Zha et al., 2013) pargle,

postoperative sore throat \&

postextubation coughing

Glycyrrhiza uralensis

In vitro LPS-induced inflammation in

glycyrrhizic acid

LPS-induced lung inflammation in mouse In vivo: $200 \mathrm{mg} /$

kg, i.p., single

dose

pathway,

ITNF- $\alpha$, IL-1 $\beta$, HMGB1 


\begin{tabular}{|c|c|c|c|c|c|}
\hline $\begin{array}{l}\text { General category } \\
\text { of therapeutic } \\
\text { activity }\end{array}$ & $\begin{array}{l}\text { Scientific name/ } \\
\text { preparation }\end{array}$ & Model/Design & $\begin{array}{l}\text { Dosage and } \\
\text { duration of } \\
\text { treatment }\end{array}$ & Mechanisms & Reference \\
\hline & $\begin{array}{l}\text { Phyllanthus emblica/EtOH } \\
\text { extract \& pyrogallol }\end{array}$ & $\begin{array}{l}\text { In vitro cytoprotective effects against } P \text {. } \\
\text { aeruginosa damage in IB3-1 bronchial } \\
\text { epithelial cells }\end{array}$ & $\begin{array}{l}500 \mu \mathrm{g} / \mathrm{ml} \text { of the } \\
\text { extract, } 2,20,200 \\
\mu \mathrm{M} \text { pyrogallol }\end{array}$ & $\begin{array}{l}\text { IIL-6, IL-8, GRO- } \alpha \text {, GRO- } \gamma, \& \text { ICAM- } 1 \text {, } \\
\text { No effect on bacterial adhesion }\end{array}$ & (Nicolis et al., 2008) \\
\hline & Rheum officinale/emodin & $\begin{array}{l}\text { In vitro TGF- } \beta 1 \text {-induced toxicity in human } \\
\text { embryo lung fibroblasts, } \\
\text { Bleomycin-induced pulmonary toxicity in } \\
\text { rat }\end{array}$ & $\begin{array}{l}\text { In vitro: } 15-60 \mu \mathrm{M}, \\
\text { In vivo: } 10-40 \mathrm{mg} / \\
\mathrm{kg} / \text { day, p.o., } 21 \\
\text { days }\end{array}$ & $\begin{array}{l}\text { In vitro: } \downarrow \alpha-S M A \text {, collagen IV, fibronectin, Smad2/3 \& STAT3 activation } \\
\text { In vivo: } \downarrow \text { Pulmonary edema \& fibrosis, TNF- } \alpha \text {, IL-6, TGF- } \beta 1, \alpha-S M A \text {, } \\
\text { HSP-47 }\end{array}$ & (Guan et al., 2016) \\
\hline & $\begin{array}{l}\text { Rheum palmatum/aqueous } \\
\text { extract }\end{array}$ & $\begin{array}{l}\text { Randomized, controlled trial in patients } \\
\text { with acute respiratory distress syndrome } \\
\text { treated with the extract+ conventional } \\
\text { drugs or only conventional drugs }\end{array}$ & $\begin{array}{l}10 \mathrm{~g} / 30 \mathrm{ml}, \mathrm{TDS} \\
\text { p.o., } 7 \text { days }\end{array}$ & $\begin{array}{l}\uparrow \text { Oxygenation, } \\
\downarrow \text { Extravascular lung water index, pulmonary vascular permeability index }\end{array}$ & (He et al., 2017) \\
\hline & $\begin{array}{l}\text { Rheum palmatum/ } \\
\text { chrysophanol }\end{array}$ & $\begin{array}{l}\text { In vitro TNF- } \alpha \text {-induced toxicity in human } \\
\text { pulmonary epithelial BEAS-2B cells, } \\
\text { OVA-induced airway inflammation in } \\
\text { mouse }\end{array}$ & $\begin{array}{l}\text { In vitro: } 2,20 \mu \mathrm{M} \\
\text { In vivo: } 0.1-10 \\
\mathrm{mg} / \mathrm{kg} / \text { day, i.p., } 4 \\
\text { days }\end{array}$ & 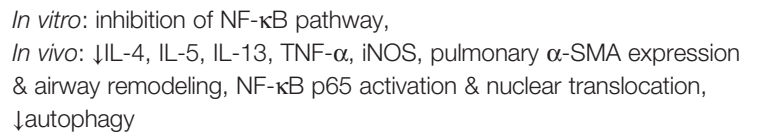 & (Song et al., 2019) \\
\hline & Rheum palmatum/rhein & $\begin{array}{l}\text { RSV-induced pulmonary damage in } \\
\text { mouse }\end{array}$ & $\begin{array}{l}30-120 \mathrm{mg} / \mathrm{kg} / \\
\text { day, p.o., } 5 \text { days }\end{array}$ & $\begin{array}{l}\text { Improvement of lung index, } \\
\downarrow \| L-1 \beta, \text { IL-6, IL-18, IL-33, TNF- } \alpha \\
\text { \NF-KB-dependent NLRP3 inflammasome activation }\end{array}$ & (Shen et al., 2019) \\
\hline & $\begin{array}{l}\text { Rosa damascena/EtOH } \\
\text { extract \& essential oil }\end{array}$ & $\begin{array}{l}\text { In vitro } \mathrm{KCl} \text {, methacholine, \& } \\
\text { methacholine + propranolol + } \\
\text { chlorpheniramine-induced contraction in } \\
\text { tracheal chains of guinea pig }\end{array}$ & $0.25-1 \%$ & $\begin{array}{l}\text { Relaxation in } \mathrm{KCl} \& \text { methacholine-induced tracheal contraction, } \\
\text { Higher activity by the essential oil vs. theophylline }\end{array}$ & $\begin{array}{l}\text { (Boskabady et al., } \\
\text { 2006) }\end{array}$ \\
\hline & $\begin{array}{l}\text { Syzygium aromaticum/ } \\
\text { aqueous extract }\end{array}$ & $\begin{array}{l}\text { In vitro cytoprotective effect on human } \\
\text { neutrophil, } \\
\text { LPS-induced lung inflammation in mouse }\end{array}$ & $\begin{array}{l}200 \mathrm{mg} / \mathrm{kg} \text {, two } \\
\text { doses, i.p. }\end{array}$ & $\begin{array}{l}\downarrow M P O \text { in neutrophils, } \\
\downarrow \text { neutrophil count, protein leakage in alveoli, MMP-2 \& -9 activity }\end{array}$ & (Chniguir et al., 2019) \\
\hline & $\begin{array}{l}\text { Syzygium aromaticum/ } \\
\text { eugenol }\end{array}$ & LPS-induced lung inflammation in mouse & 160 mg/kg, i.p. & $\begin{array}{l}\text { Improvement of lung function, } \\
\text { \alveolar collapse, collagen fibers, \& neutrophil influx, } \\
\downarrow \text { NF-KB activation \& TNF- } \alpha\end{array}$ & (Magalhaes et al., 2010) \\
\hline & $\begin{array}{l}\text { Trigonella foenum-graecum/ } \\
\text { hot water extract as syrup }\end{array}$ & $\begin{array}{l}\text { Randomized controlled trial in patients } \\
\text { with mild asthma }\end{array}$ & $\begin{array}{l}10 \mathrm{ml}, \mathrm{BD}, 4 \\
\text { weeks }\end{array}$ & $\begin{array}{l}\text { Improvement of spirometry parameters \& quality of life, } \\
\downarrow \| \mathrm{L}-4\end{array}$ & (Emtiazy et al., 2018) \\
\hline & $\begin{array}{l}\text { Trigonella foenum-graecum/ } \\
\text { hydroalcoholic extract }\end{array}$ & $\begin{array}{l}\text { Bleomycin-induced pulmonary toxicity in } \\
\text { rat }\end{array}$ & $\begin{array}{l}5-40 \mathrm{mg} / \mathrm{kg} / \text { day, } \\
\text { p.o., } 28 \text { days }\end{array}$ & 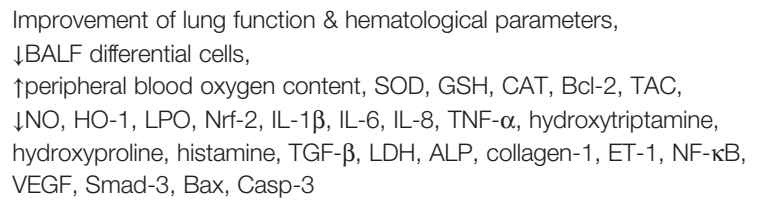 & (Kandhare et al., 2015) \\
\hline & $\begin{array}{l}\text { Vitis vinifera/polyphenolic } \\
\text { extract }\end{array}$ & $\begin{array}{l}\text { Bleomycin-induced pulmonary toxicity in } \\
\text { mouse }\end{array}$ & $\begin{array}{l}50,100 \mathrm{mg} / \mathrm{kg} / \\
\text { day, p.o., } 21 \text { days }\end{array}$ & $\begin{array}{l}\downarrow \text { Leukocytes infiltration in BALF, hydroxyproline, TGF- } \beta 1, \text { MMP-9, } \\
\text { collagen } 1-\alpha 1 \text {, fibronectin- } 1 \text {, } \\
\uparrow E-\text { cadherin }\end{array}$ & (Liu et al., 2017) \\
\hline & $\begin{array}{l}\text { Vitis vinifera/proanthocyanidin } \\
\text { extract }\end{array}$ & $\begin{array}{l}\text { In vitro RSV-induced inflammation in } \\
\text { human airway epithelial A549 cells }\end{array}$ & $5,10 \mu \mathrm{g} / \mathrm{ml}$ & $\downarrow\|L-1 \beta\| L-6,, \| L-8$ mRNA \& protein expression & (Kim et al., 2019) \\
\hline & $\begin{array}{l}\text { Vitis vinifera/proanthocyanidin } \\
\text { extract }\end{array}$ & $\begin{array}{l}\text { In vitro As-induced toxicity in human lung } \\
\text { epithelial BEAS-2B cells, } \\
\text { As-induced pulmonary toxicity in mouse }\end{array}$ & $\begin{array}{l}\text { In vitro: } 25,50 \\
\mathrm{mg} / \mathrm{ml} \\
\text { In vivo: } 400 \mathrm{mg} / \\
\mathrm{kg} / \text { day, p.o., } 5 \\
\text { weeks }\end{array}$ & $\begin{array}{l}\text { In vitro \& in vivo: } \\
\downarrow \text { Apoptosis, } L P O, R O S,\|L-1 \beta,\| L-6, C R P, T N F-\alpha, N F-\kappa B \text { activation, } \\
\uparrow 1 L-10, \\
\text { llung inflammation \& edema in vivo }\end{array}$ & (Hu et al., 2019) \\
\hline
\end{tabular}




\section{General category}

Model/Design

Dosage and

duration of

treatment

activity

Vitis vinifera/proanthocyanidin $\mathrm{Pb}$-induced pulmonary toxicity in rat extract

$200 \mathrm{mg} / \mathrm{kg} / \mathrm{day}$,

p.o., 5 weeks

$\uparrow$ AMPK/Nrf2/p62 signaling activation,

Mechanisms

Reference

Vitis vinifera/proanthocyanidin Carrageenan-induced pulmonary inflammation in mouse

$25-100 \mathrm{mg} / \mathrm{kg}$, extract

p.o., single dose

$\downarrow \mathrm{Pb}$ pulmonary concentration, apoptosis, LPO, Bax, p53, TNF- $\alpha$, NF-

кB nuclear translocation, $\mathrm{HO}-1$

Nephroprotective Allium sativum/diallyl trisulfide As-induced nephrotoxicity in rat

$80 \mathrm{mg} / \mathrm{kg} /$ day,

IIL-17A \& GITR expressing cells,

IIL-17A, IL-1 $\beta$, IL-2, IL-6, IL-12, IFN $\gamma$, ICAM-1, TNF- $\alpha$, MCP-1,

$\uparrow T G F-\beta 1$, IL-4, IL5, IL-10

activity

Allium sativum/S-

allylmercaptocysteine

In vitro cisplatin-induced cytotoxicity in human kidney HK-2 cells,

Cisplatin-induced nephrotoxicity in rat

Alpinia galanga/galangin

Cichorium intybus/Aqueous extract

Cichorium intybus/Aqueous extract

Crocus sativus/aqueous

extract

Crocus sativus/crocin

Crocus sativus/crocin

Glycyrrhiza spp./glycyrrhizic acid

Phyllanthus emblical

emblicanin-A \& -B enriched

extract

Rheum officinale/different

extracts
High-fructose diet-induced nephrotoxicity in rat

In vitro cytoprotection in HCK cells,

Adenine + yeast-induced chronic kidney disease in rat

STZ-induced diabetic nephropathy in rat EtOH-induced nephrotoxicity in rat

Tartrazine-induced nephrotoxicity in rat

STZ-induced diabetic nephropathy in rat

vitro LPS-induced toxicity in rat mesangial cells,

LPS-induced nephrotoxicity in rat

Cisplatin-induced nephrotoxicity is rat

Adenine-induced chronic kidney disease in rat p.o., 28 days

IBUN, $\mathrm{Cr}$

renal As concentration, membranes bound ATPases, Bax, Cyt C, Nox2, p47phox \& Nox4, TNF- $\alpha$, IL-1 $\beta$, IL-6, iNOS, NF-kB, Casp-3, 个renal SOD, CAT, GST, Gpx, GR, G6PD, GSH, TSH, vitamin C \& E, $\uparrow A k t$, PI3K \& their phosphorylated form, Bcl-2

In vitro: 50-100 In vitro: \apoptosis, cleaved PARP, p53,

$\mu \mathrm{M}, \quad \begin{aligned} & \uparrow c l-2, \\ & \end{aligned}$ $\mathrm{kg} / \mathrm{day}$, i.p., $20 \quad \uparrow \mathrm{Nr}$ 2, NQO1, CAT, SOD, GSH

days

$50-200 \mu \mathrm{g} / \mathrm{kg} / \quad \downarrow L P O$, Micro-albuminuria \& tubular glomerular damage,

day, P. 0,60 days $\uparrow$ renal \& plasma SOD, CAT, Gpx, GSH, vitamin C \&

In vitro: 100-400 In vitro: \transmembrane transport of uric acid,

In vivo: $\downarrow$ serum uric acid \& Cr, microalbuminuria, GLUT-9 protein

$\mu \mathrm{g} / \mathrm{ml}$

In vivo: 6.6, 13.2 expression

weeks

$125 \mathrm{mg} / \mathrm{kg} /$ day,

i.p., 21 days

$40-160 \mathrm{mg} / \mathrm{kg}$

day, p.o., 4 weeks

$50 \mathrm{mg} / \mathrm{kg} /$ day,

p.o., 21 days

$20 \mathrm{mg} / \mathrm{kg} /$ day,

p.o., 21 days

In vitro: 50, 100

$\mu \mathrm{M}$,

In vivo: 25,50

$\mathrm{mg} / \mathrm{kg}$, i.p., single In vivo: $\downarrow$ BUN, Cr, TNF- $\alpha$, MCP-1, ICAM-1, VCAM-1

dose

150-600 mg/kg/ $\uparrow$ Renal CAT, GSH, SOD,

day, p.o., 10 days $\downarrow$ Inflammation \& apoptosis,

ILPO, MAPK phosphorylation

200-800 mg/kg/ \Renal $\alpha$-SMA, collagen-I \& collagen-III,

day, p.o., 6 weeks $\downarrow$ BUN, Cr, TGF- $\beta 1$, TGF- $\beta$ receptor I \& II, Smad-2, Smad-3, Smad4,

vimentin

$\uparrow S m a d 7$, E-cadherin
(Lu et al., 2018)

(Ahmad et al., 2014)

(Miltonprabu et al.,

2017)

(Zhu et al., 2017)

(Sivakumar et al., 2010)

(Jin et al., 2018)

(Pourfarjam et al., 2017)

(Rezaee-Khorasany

et al., 2019)

(Erdemli et al., 2017)

(Altinoz et al., 2015)

(Zhao et al., 2016)

(Malik et al., 2016)

(Zhang et al., 2018)

(Tu et al., 2017) 


\section{General category}

Model/Design

Dosage and

duration of

Mechanisms

Reference

of therap

treatment

Rheum palmatum/aqueous

$1 \mathrm{~g} / \mathrm{kg} /$ day, p.o., In vivo: $\downarrow$ Renal fibrosis, collagen-1, fibtonectin, LC3 conversion by the

Vitis vinifera/proanthocyanidin In vitro glucosamine-induced extract, rhein

Syzygium aromaticum aqueous extract Trigonella foenum-graecum/ STZ-induced diabetic nephropathy in rat seed powder

In vitro Hank's balanced salt solutioninduced autophagy in NRK-52E normal rat kidney cells,

Adenine-induced chronic kidney disease

In vitro: $1,10 \mu \mathrm{M}$,

In vivo: in rat

Infectious pyelonephritis in rat

$500 \mathrm{mg} / \mathrm{kg} / \mathrm{day}$ p.o., 28 days

$10 \%$ of the diet

weight, 6 weeks

B2

nephrotoxicity in rat mesangial cells

Vitis vinifera/proanthocyanidin STZ-induced diabetic nephropathy in rat extract
2.5, $10 \mu \mathrm{g} / \mathrm{m}$

$250 \mathrm{mg} / \mathrm{kg} /$ day

p.o., 16 weeks

$400 \mathrm{mg} / \mathrm{kg} / \mathrm{day}$

p.o., 5 weeks
Vitis vinifera/proanthocyanidin Diatrizoate-induced nephrotoxicity in rat $100 \mathrm{mg} / \mathrm{kg} / \mathrm{day}, \quad \downarrow \mathrm{BUN}, \mathrm{Cr}, \mathrm{Casp}-1$ \& -3 , calpain-1, iNOS, eNOS,

In vitro: \autophagy via AMPK-dependent mTOR signaling pathway,

Erk \& p38 MAPKs by rhein

extract

Leukocyte count,

Normalization of histomorphological changes

$\downarrow$ Renal Glut-1 \& -2, ACE, iNOS, \& NO,

ouabain-sensitive, $\mathrm{Mg}^{2+}$ ATPase, \& $\mathrm{Ca}^{2+}$ ATPase, fructose 1,6

diphosphatase, G6 Pase, LDH activity,

†renal hexokinase \& G6PD activity,

lurinary excretion of proteins,

$\downarrow$ renal polyol pathway enzyme activity,

$\downarrow$ podocyte damage \& morphological changes

$\downarrow$ Apoptosis \& mitochondrial dysfunction,

$\uparrow G p x$, SOD, PGC- $1 \alpha$, SIRT1, AMPK, NRF1,

No significant change in $\mathrm{BUN} \& \mathrm{Cr}$,

Irenal index, urinary albumin, endoplasmic reticulum stress-induced

apoptosis via Casp-12

$\uparrow I L-10$

extract

Better effect than $\mathrm{N}$-acetylcysteine
(Bao et al., 2015)

(Nassan et al., 2015)

(Pradeep et al., 2019)

(Gao et al., 2018)

(Wang C. et al., 2017)

(Ulusoy et al., 2014)
Irenal AST, ALT, alkaline \& acid phosphatases, $\mathrm{Na}^{+}, \mathrm{K}^{+}$,

INF-kB activation, IL-1 $\beta$, IL-6, CRP, TNF- $\alpha$,

SOD, superoxide dismutase; CAT, catalase; Gpx, glutathione peroxidase; GSH, glutathione; GR, glutathione reductase; GST, glutathione-S-transferase; LPO, lipid peroxidation; p.o., oral; I/R, ischemia-reperfusion; HIV, human

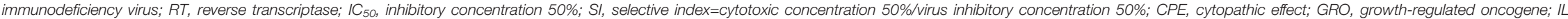
interleukin: ICAM, intercelluler adhesion molecule: CK-MB: ISO, isoproterenol: STZ streptozotocin. Cr creatinine; BUN, blood urea nitrogen: IFN, interferon: TAC, total antioxidant capacity: NO, nitric oxide; iNOS, inducible nitric oxide synthase; eNOS, endothelial nitric oxide synthase; LPS, lipopolysaccharide; MPO, myeloperoxidase; MMP. matrix metalloproteinase; PG, prostaglandin; COX, cyclooxygenase; STAT. signal transducer and activator of transcription; NF-kB, nuclear factor- KB: WBC, white blood cells; LDH, lactate dehydrogenase; CPK, creatinine phosphokinase; AST, aspartate transaminase; ALT, alanine transaminase; G6PD, glucose 6 phosphate dehydrogenase; LDL, low-density lipoprotein nuclear factor- kB; WBC, white blood cells; LDH, lactate dehydrogenase; CPK, creatinine phosphokinase; AST, aspartate transaminase; ALT, alanine transaminase; G6PD, glucose 6 phosphate dehydrogenase; LDL, low-density lipoprotein
cholesterol; TAG, triacylglycerol; ACE, angiotensin converting enzyme; NPSH, Non protein thiol; RAS, renin angiotensin system; PUFA, poly unsaturated fatty acids; SFA, saturated fatty acid; lg, immunoglobulin; Casp, caspase; SRBC, sheep cholesterol; TAG, triacylglycerol; ACE, angiotensin converting enzyme; NPSH, Non protein thiol; RAS, renin angiotensin system; PUFA, poly unsaturated fatty acids; SFA, saturated fatty acid; lg, immunoglobulin; Casp, caspase; SRBC, sheep
red blood cells; DOX, doxorubicin; DNP, dinitrophenyl; HO, heme oxygenase; MCP-1, monocyte chemoattractant protein; VCAM, vascular cell adhesion molecule; RSV, respiratory syncytial virus; BALF, bronchoalveolar lavage fluids; TLR, Toll-like receptor; NQO1, NAD(P)H:quinone oxidoreductase 1; PI3K, phosphatidylinositol-3 kinase; CREB, CAMP response element binding; GSK-3a/b, glycogen synthase kinase 3a/b; ERK, extracellular signal-regulated kinase; As, arsenic TSH, total sulfhydryl groups; CRP, C-reactive protein; Pb, lead; gGCS, g-glutamylcysteine synthetase; GRP78, glucose-regulated protein 78; PERK, protein kinase RNA-like ER kinase; elF2a, eukaryotic translation initiation factor-2; JNK, CJun N-terminal kinase; AP-1, activator protein-1; CXCL10, interferon-g-inducible protein 10; OVA, ovalbumin; EV, enterovirus; mTOR, mammalian target of rapamycin; RDDP, Reverse Transcriptase-associated DNA Polymerase; RNase H, Ribonuclease H: a-SMA, asmooth muscle actin; STAT signal transducer and activator of transcription; HSP, heat shock protein: NOX4, NADPH oxidase 4; PPAR, Poly(ADP) ribose polymerase; HMGB, High Mobility Group Box.

Bold studies are antiviral assessments against viral lung pathogens. 
TABLE 3 | Quality assessment of animal studies on the pharmacological activity of traditional Persian medicine-suggested plant possibly beneficial in COVID-19 according to Animal Research: Reporting of In vivo Experiments (ARRIVE) guideline.

\begin{tabular}{|c|c|c|c|c|c|c|c|c|}
\hline Reference & Validity & $\begin{array}{c}\text { Ethical } \\
\text { statement }\end{array}$ & Animals & $\begin{array}{l}\text { Experimental } \\
\text { procedures }\end{array}$ & $\begin{array}{l}\text { Housing \& } \\
\text { husbandry }\end{array}$ & $\begin{array}{l}\text { Numbers } \\
\text { analyzed }\end{array}$ & $\begin{array}{l}\text { Interpretation } \\
\text { \& scientific } \\
\text { implications }\end{array}$ & $\begin{array}{c}\text { Generalizability/ } \\
\text { translation }\end{array}$ \\
\hline (Sawamura et al., 2010) & - & + & + & + & + & + & - & - \\
\hline (Xiong et al., 2012) & - & + & + & + & - & + & - & - \\
\hline (Hong et al., 2015) & + & + & + & + & - & - & - & - \\
\hline (Abdel-Daim et al., 2017) & + & + & + & + & + & + & - & + \\
\hline (Zhao et al., 2019) & + & + & + & + & - & + & - & + \\
\hline (Yu et al., 2017) & & + & + & + & + & + & - & + \\
\hline (Padiya et al., 2014) & - & + & + & + & + & + & - & - \\
\hline (Qi et al., 2020) & + & + & + & + & + & + & - & + \\
\hline (Sachdeva et al., 2012) & + & + & + & + & + & + & - & + \\
\hline (Mehdizadeh et al., 2013) & + & - & + & + & + & + & - & - \\
\hline (Razmaraii et al., 2016) & + & + & + & + & + & + & - & + \\
\hline (Ojha et al., 2013) & + & + & + & + & + & + & - & + \\
\hline (Li et al., 2019) & + & + & - & + & + & + & - & + \\
\hline (Thirunavukkarasu et al., 2015) & + & + & + & + & - & - & - & - \\
\hline (Bhattacharya et al., 2002) & - & - & + & + & + & + & - & + \\
\hline (Ojha et al., 2012) & + & + & + & + & + & + & - & + \\
\hline (Patel and Goyal, 2011) & + & + & + & + & + & + & - & + \\
\hline (Lu et al., 2019) & + & + & + & + & - & + & - & + \\
\hline (Panda and Kar, 2010) & + & + & + & + & + & + & - & - \\
\hline (Feki et al., 2019) & + & + & + & + & + & + & - & - \\
\hline (Mukthamba and Srinivasan, 2015) & + & + & + & + & + & + & - & - \\
\hline (Tripathi and Chandra, 2009) & + & + & + & + & + & + & - & + \\
\hline (Pradeep and Srinivasan, 2018) & + & + & + & + & + & + & - & - \\
\hline (Panda et al., 2013) & + & + & + & + & + & + & - & - \\
\hline (Ammar el et al., 2013) & + & + & + & + & + & + & - & + \\
\hline (Cheng et al., 2012) & + & + & + & + & + & + & - & - \\
\hline (Kim et al., 2002) & + & - & + & + & + & + & - & - \\
\hline (Lepczynski et al., 2015) & + & + & + & + & - & - & - & - \\
\hline (Ramadan et al., 2011) & + & + & + & + & - & - & - & + \\
\hline (Long et al., 2016) & + & + & + & + & + & + & - & - \\
\hline (Zou et al., 2018) & - & + & + & + & + & - & - & + \\
\hline (Mohi El-Din et al., 2014) & + & + & + & + & + & - & - & - \\
\hline (Nie et al., 2019) & + & + & + & + & - & + & - & + \\
\hline (Mo et al., 2020) & + & + & + & + & + & + & - & + \\
\hline (Seo et al., 2013) & - & + & - & + & - & + & - & + \\
\hline (Shu et al., 2014) & - & + & + & + & + & + & - & - \\
\hline (Zha et al., 2013) & - & + & + & + & + & + & - & + \\
\hline (Zhang et al., 2020b) & - & + & + & + & + & + & - & + \\
\hline (Zaghloul et al., 2019) & + & + & + & + & - & + & - & + \\
\hline (Boskabady et al., 2014) & + & + & + & + & - & + & - & + \\
\hline (Ma et al., 2013) & + & + & + & + & - & + & - & + \\
\hline (Nishimoto et al., 2010) & - & + & + & + & - & + & - & + \\
\hline (Qu et al., 2019) & + & + & + & + & - & + & - & + \\
\hline (Guan et al., 2016) & + & + & + & + & + & + & - & + \\
\hline (Song et al., 2019) & - & + & + & + & + & - & - & + \\
\hline (Shen et al., 2019) & + & + & + & + & + & + & - & + \\
\hline (Chniguir et al., 2019) & - & + & + & + & - & + & - & - \\
\hline (Magalhaes et al., 2010) & + & + & + & + & - & + & - & + \\
\hline (Kandhare et al., 2015) & + & + & + & + & + & + & - & - \\
\hline (Liu et al., 2017) & + & + & + & + & + & + & - & + \\
\hline (Hu et al., 2019) & + & - & - & + & - & + & - & + \\
\hline (Lu et al., 2018) & + & + & + & + & + & + & - & + \\
\hline (Ahmad et al., 2014) & + & + & + & + & + & + & - & - \\
\hline (Miltonprabu et al., 2017) & + & + & + & + & + & + & - & + \\
\hline (Zhu et al., 2017) & + & + & + & + & + & + & - & + \\
\hline (Sivakumar et al., 2010) & + & + & + & + & + & + & - & - \\
\hline (Jin et al., 2018) & + & + & + & + & + & + & - & - \\
\hline (Pourfarjam et al., 2017) & + & + & + & + & + & - & - & - \\
\hline (Rezaee-Khorasany et al., 2019) & + & + & + & + & + & + & - & - \\
\hline (Erdemli et al., 2017) & + & + & + & + & + & + & - & + \\
\hline
\end{tabular}


TABLE 3 | Continued

\begin{tabular}{|c|c|c|c|c|c|c|c|c|}
\hline Reference & Validity & $\begin{array}{c}\text { Ethical } \\
\text { statement }\end{array}$ & Animals & $\begin{array}{c}\text { Experimental } \\
\text { procedures }\end{array}$ & $\begin{array}{l}\text { Housing \& } \\
\text { husbandry }\end{array}$ & $\begin{array}{l}\text { Numbers } \\
\text { analyzed }\end{array}$ & $\begin{array}{l}\text { Interpretation } \\
\text { \& scientific } \\
\text { implications }\end{array}$ & $\begin{array}{c}\text { Generalizability/ } \\
\text { translation }\end{array}$ \\
\hline (Altinoz et al., 2015) & + & + & + & + & + & - & - & - \\
\hline (Zhao et al., 2016) & + & + & + & + & + & + & - & - \\
\hline (Malik et al., 2016) & + & + & + & + & + & + & - & + \\
\hline (Zhang et al., 2018) & + & + & + & + & - & + & - & - \\
\hline (Tu et al., 2017) & - & + & + & + & + & - & - & + \\
\hline (Nassan et al., 2015) & - & - & + & + & + & - & - & + \\
\hline (Pradeep et al., 2019) & + & + & + & + & + & + & - & + \\
\hline (Gao et al., 2018) & + & + & + & + & - & + & - & + \\
\hline (Wang C. et al., 2017) & + & + & + & + & + & + & - & + \\
\hline (Ulusoy et al., 2014) & + & + & + & + & + & - & - & + \\
\hline
\end{tabular}

GSK3 $\beta$ ) pathway, was identified in the $\mathrm{I} / \mathrm{R}$ model. The phosphorylation of Akt and subsequently GSK3 $\beta$ leads to the release of 1-methyl-4-phenyl-1,2,3,6-tetrahydropyridine and $\beta$ catenin nuclear translocation which further activates antiapoptotic signaling pathways like B cell lymphoma-2 (Bcl-2) and endothelial Nitric oxide synthase (eNOS), finally resulting in cardioprotection (Thirunavukkarasu et al., 2015).

The effective animal dosage of amla is $100-500 \mathrm{mg} / \mathrm{kg} /$ day for the aforementioned therapeutic activities. This dose is equal to a relatively high human dose; however, the plant has an acceptable safety profile and is routinely taken in several countries of the world. Thus, these studies suggest that amla could be a functional food, useful for primary and secondary prevention of COVID-19 (Pingali Usharani and Muralidhar, 2013; Upadya et al., 2019; Kapoor et al., 2020). Interestingly, amla fruit is the second richest source of vitamin C (nearly 600-700 $\mathrm{mg}$ in each fruit), which WHO has recommended people take to protect the immune system against SARS-CoV-2 infection (Goraya and Bajwa, 2015).

\section{Chicory (Cichorium intybus L.)}

Chicory is a cosmopolitan herbaceous plant from the Compositae (Asteraceae) family, used both as a medicinal plant and a food additive because it tastes similar to coffee. All parts of the plant, including roots, aerial parts, and seeds, are used due to its medicinal properties. Chicory is well-known as a hepatoprotective plant in different complementary and alternative medicines (Street et al., 2013). In TPM, it is also considered as a tonic for kidney and heatrelated heart diseases, as well as a modulator of overall health through prevention of humor infection.

Chicory extract was effective in the prevention of diabetic nephropathy following three weeks of administration to rats. At the dose of $125 \mathrm{mg} / \mathrm{kg}$, the effect of chicory was approximately equal to $100 \mathrm{mg} / \mathrm{kg}$ of metformin in several parameters (Pourfarjam et al., 2017). The nephroprotective effect of the extract was confirmed in the human kidney cell line through inhibition of GLUT-9 expression, an important transporter of uric acid in kidneys. Chicory extract could also decrease renal damage in an experimental model of chronic renal failure; however, the administered doses of the extract (6.6 and $13.2 \mathrm{~g} /$ $\mathrm{kg}$ ) were dramatically higher than benzbromarone $(20 \mathrm{mg} / \mathrm{kg})$ as the standard drug (Jin et al., 2018).
Chicory is also demonstrated to have immunomodulatory activities. In the animal model of ethanol-induced immunotoxicity, chicory increased both circulating leukocytes and the weight of lymphatic organs, showing an improvement in immune system function (Kim et al., 2002). Additionally, chicory as a dietary supplement could affect the plasma protein profiles, resulting in the lower level of pro-inflammatory markers such as the C-reactive protein (CRP) (Lepczynski et al., 2015). Chicory root is a rich source of inulin-type fructans, a group of carbohydrates considered as prebiotics (Lepczynski et al., 2015). Today, the remarkable role of the normal flora of different body organs in various diseases, including immunological problems, has attracted the attention of scientists (Pretorius et al., 2018). Thus, aside from the direct immunomodulatory effects of chicory, the presence of such prebiotics in this plant may also have a modulatory effect on normal flora. The indirect protective effect of chicory against pathologic conditions can be hypothesized in future studies.

\section{Clove (Syzygium aromaticum (L.) Merr. \& L.M.Perry)}

The flower buds of clove, from the family Myrtaceae, have long been used in both medicine and for culinary purposes. The phenylpropanoids of the essential oil, mainly eugenol, are considered to be the main active compounds of the plant and are responsible for several pharmacological activities (Chaieb et al., 2007). Clove is known as a tonic for the cardiovascular system in TPM and is thought to improve blood supply to both the heart and the brain. Moreover, it is mentioned to be specifically useful in chronic coughs, shortness of breath, and palpitations (Aghili Khorasani, 1771).

The methanolic and aqueous extracts of clove have shown inhibitory effects on $\mathrm{HCV}$ protease enzyme with an $\mathrm{IC}_{50}$ of 33 $\mu \mathrm{g} / \mathrm{ml}$ (Hussein et al., 2000). Eugenol has exhibited antiviral effects against the influenza A virus through direct reduction of viral replication, as well as inhibition of autophagy, a supporting mechanism for viral replication and cell death which results in acute lung damage. It should be mentioned that the potency of eugenol at the concentration of $5 \mu \mathrm{g} / \mathrm{ml}$ was equal or higher than $25 \mu \mathrm{g} / \mathrm{ml}$ of ribavirin as the gold standard antiviral agent, recommending eugenol as a potent antiviral compound 
(Dai et al., 2013). Furthermore, eugenol could regulate cellular inflammatory cascades such as nuclear factor- $\kappa \mathrm{B}(\mathrm{NF}-\kappa \mathrm{B})$ and extracellular signal-regulated kinase (ERK)/mitogen-activated protein kinase (MAPK) pathways, nitric oxide (NO), the release of pro-inflammatory ILs, and endogenous antioxidant defense mechanisms (Table 2).

Clove has demonstrated modulatory effects on the function of murine white blood cells and macrophages damaged through inflammation/oxidative stress damage (Table 2). In the animal model of immunosuppression, one-week administration of clove essential oil (400 mg/kg) could improve both humoral and cellmediated immunity with equal efficacy to $50 \mathrm{mg} / \mathrm{kg}$ of levamisole (Carrasco et al., 2009). Clove extract and its major ingredient, eugenol, have shown an anti-inflammatory effect on the lipopolysaccharide (LPS)-induced damage in macrophages. No statistically significant difference was observed between eugenol (100 $\mu \mathrm{g} /$ well $)$ and dexamethasone $\left(10^{-4} \mathrm{~mol} / \mathrm{l}\right)$ (Bachiega et al., 2012). The same effect is also reported with a single dose of the flavonoid biflorin (Lee et al., 2016), suggesting this compound is a fast-acting agent that may be useful in acute inflammations such as cytokine storm in COVID-19.

LPS-induced lung inflammation was also relieved with clove aqueous extract and eugenol through reduction of tumor necrosis factor (TNF)- $\alpha$ and inhibition of NF- $\kappa B$ signaling, as well as improvement in alveolar damage (Magalhaes et al., 2010; Chniguir et al., 2019).

Moreover, the aqueous extract has demonstrated a protective effect on an animal model of infectious pyelonephritis (Nassan et al., 2015), a condition also reported in COVID-19 patients ( $\mathrm{Su}$ et al., 2020).

Aside from the above-mentioned features of clove essential oil, it has shown strong antibacterial effects, even against the infections of immunosuppressed hospitalized patients (Chaieb et al., 2007). Thus, the essential oil can be a valuable option to prevent secondary bacterial infections in COVID-19 patients.

\section{Damask Rose (Rosa x damascena Herrm.)}

Damask rose, from the family Rosaceae, is one of the most valued medicinal plants in TPM due to its modulatory effects on the function of almost all body organs and consequently, overall health (Nayebi et al., 2017). It helps the body to excrete abnormal watery phlegm humor which is highly susceptible to infection and thus, it is a tonic of the lungs. Furthermore, TPM texts discuss that it alleviates infectious fevers (Aghili Khorasani, 1771).

An in vitro study on the antiviral properties of damask rose has demonstrated significant activity against HIV infection. Flavonoids purified from the methanolic extract including quercetin, kaempferol, and two of its analogues, showed the highest activity via the inhibition of viral protease and gp 120 binding to CD4 glycoprotein. The compounds were not as potent as azidothymidine (zidovudine) as a standard anti-HIV agent in regard to SI and $\mathrm{IC}_{50}$; however, they showed an additive effect in combination with this drug (Mahmood et al., 1996).

Damask rose has shown cardiotonic properties on perfused guinea pig heart and reversed bradycardia by increasing heart contractility (Boskabady et al., 2013). Both ethanolic extract and the essential oil represented an antispasmodic effect on guinea pig tracheal chains (Boskabady et al., 2006).

\section{Fenugreek (Trigonella foenum-graecum L.)}

Fenugreek is a member of the family Leguminosae and the seeds are frequently used as a lung tonic in TPM due to the moderate heat, causing a mucolytic activity on pulmonary mucosa. This effect helps to remove the thick phlegm humor and causes a soothing effect on lung injuries, accelerating the healing procedure (Aghili Khorasani, 1771).

Fenugreek seed extract has demonstrated significant antiinflammatory properties in an animal model of pulmonary inflammation, evident from the reduction of leukocytes infiltration to the bronchoalveolar lavage fluid (BALF) and lung fibrosis. Pro-inflammatory cytokines and endogenous enzymatic and non-enzymatic antioxidants were also restored to near normal levels. Stimulation of nuclear factor E2-related factor 2 (Nrf2) by fenugreek, an antioxidant cascade, prevents the hemeoxigenase-1 (HO-1) overproduction, subsequently ameliorating pulmonary fibrosis. The effectiveness of the extract with $5-40 \mathrm{mg} / \mathrm{kg}$ dose range in most of the investigated parameters were equal or higher than $10 \mathrm{mg} / \mathrm{kg}$ of methylprednisolone as the gold standard drug (Kandhare et al., 2015). Likewise, the antiasthmatic effect of fenugreek seed syrup, designed based on the lung protecting properties of the plant in TPM, was assessed in a clinical trial. Four weeks of treatment with the syrup caused a significant improvement in spirometry parameters in comparison to the baseline values (Emtiazy et al., 2018).

There are also several studies on the cardioprotective effects of fenugreek (Table 2). Dietary fenugreek powder (Mukthamba and Srinivasan, 2015), trigonelline (a pyridine alkaloid), and digoxigenin-3-O-rutin (a cardiac glycoside) have reversed isoproterenol-induced cardiotoxicity in rats (Panda and Kar, 2010; Panda et al., 2013). Both compounds exerted modulatory effects on the cardiac biomarkers of oxidative stress and inflammation, including $\mathrm{CK}-\mathrm{MB}$, lactate dehydrogenase (LDH), and lipid peroxidation. Trigonelline showed the highest pharmacological activity at a dose of $40 \mathrm{mg} / \mathrm{kg}$. On the other hand, digoxigenin-3-O-rutin was effective at $2.5-10 \mathrm{mg} / \mathrm{kg}$ which was comparable with $5 \mathrm{mg} / \mathrm{kg}$ of digoxin as the gold standard drug (Panda and Kar, 2010). Furthermore, trigonelline could reduce the level of heat shock protein (HSP)-27 and $\alpha \mathrm{B}$ crystallin, two novel biomarkers of oxidative damage, in the myocardium damage, further confirming the cardioprotective activity of fenugreek (Panda et al., 2013). Polysaccharides as another important category of fenugreek components have demonstrated significant cardioprotective activity both in vitro and in vivo, possibly due to their antioxidant activity and prevention of DNA damage (Feki et al., 2019). Aside from antioxidant activity (Tripathi and Chandra, 2009), inhibition of pathologic NO production by inducible nitric oxide synthase (iNOS) and markers of cardiac fibrosis, such as fibronectin and collagen, seem to be the main cardioprotective mechanisms of fenugreek demonstrated in streptozotocin (STZ)-induced cardiac damage (Pradeep and Srinivasan, 2018). 
In a cyclophosphamide-induced animal model of immunosuppression, fenugreek could prevent lymphopenia and neutropenia, and improve the cellularity of bone marrow, spleen, and thymus, proposing an immunostimulatory effect for this plant (Ramadan et al., 2011).

\section{Galangal (Alpinia galanga (L.) Willd., A. officinarum Hance)}

Galangal species belong to the family Zingiberaceae, a valuable plant family comprising of several important medicinal plants such as ginger and turmeric (Abubakar et al., 2018). In TPM, the plant is known to have nephroprotective properties and lung tonic activity and is used for the treatment of cough. Aside from the essential oil, the most important secondary metabolites in this plant family are diarylheptanoids with significant antiinflammatory properties (Abubakar et al., 2018).

Seven diarylheptanoids from lesser galangal (A. officinarum) have demonstrated significant in vitro antiviral effects on RSV, poliovirus, and measles virus. The lowest $\mathrm{IC}_{50}$ values were 5, 3.7, and $6.3 \mu \mathrm{g} / \mathrm{ml}$ against RSV, poliovirus, and measles virus, respectively; however, they were all higher than those of the gold standards, ribavirin and acyclovir (Konno et al., 2011). In another study, the antiviral effects of two galangal diarylheptanoids were assessed against several types of influenza virus, one of which showed remarkable activity. The active compound was not only effective in vitro against all virus types, including oseltamivir-resistant type, but also showed in vivo protective effects on the murine model of influenza. The compound showed a dose-dependent inhibition of viral RNA and antigen expression; while it was ineffective on the viral adsorption or invasion. The in vitro inhibition of viral growth by $60 \mu \mathrm{g} / \mathrm{ml}$ of the compound was higher than $20 \mu \mathrm{g} / \mathrm{ml}$ of ribavirin (Sawamura et al., 2010). 1'-Acetoxychavicol acetate, a phenylpropanoid from the greater galangal (A. galanga) also exhibited anti-influenza activity with an $\mathrm{IC}_{50}$ of $2 \mu \mathrm{M}$ (460 ng/ $\mathrm{ml}$ ). This effect was mediated through the inhibition of viral ribonucleoprotein complex nuclear export, an important part of the viral life cycle that controls the transcription and replication (Watanabe et al., 2011). Furthermore, the compound has demonstrated an inhibitory effect on the nuclear export of HIV-Rev protein. Analysis of the structure-activity relationship revealed that the presence of 10-acetoxyl-20-ene moiety, two acetyl functional groups, along with a 10-S configuration is crucial for its antiviral activity (Tamura et al., 2009). It is worth mentioning that a molecular docking analysis showed the effectiveness of galangal compounds against SARS-CoV-2; however, experimental studies are needed to confirm this hypothesis (Zhang et al., 2020a).

1 '-Acetoxychavicol acetate has been proved to have a protective effect on the lungs, as well. In an ovalbumininduced mouse model of asthma, the compound reduced the infiltration of eosinophils into the BALF. The secretion of proinflammatory cytokines by both types 1 and $2 \mathrm{~T}$ cells was also significantly decreased and the effect of the higher dose $(50 \mathrm{mg} /$ $\mathrm{kg}$ ) was equal to $1 \mathrm{mg} / \mathrm{kg}$ of dexamethasone (Seo et al., 2013). Galangin, a flavonoid of the greater galangal, has represented anti-inflammatory effects in the same animal model via inhibition of the NF- $\kappa \mathrm{B}$ pathway. Furthermore, galangin decreased the monocyte chemoattractant protein (MCP)-1 and vascular cell adhesion molecule (VCAM)-1 of lung tissue, both of which participate in leukocytes chemotaxis (Zha et al., 2013). It should be mentioned that in most evaluated parameters, the effect of $15 \mathrm{mg} / \mathrm{kg}$ of galangin was equal or higher than dexamethasone $(3 \mathrm{mg} / \mathrm{kg})$, showing a high anti-inflammatory potency (Zha et al., 2013). Moreover, galangin has demonstrated anti-inflammatory effects in LPS-induced acute lung damage (Shu et al., 2014) and macrophage stimulation (Jung et al., 2014) through the suppression of NF- $\kappa B$ downstream signaling.

In addition to galangin, 1 '-acetoxychavicol acetate has also shown immunoregulatory properties in stimulated murine macrophages via the prevention of NF- $\mathrm{KB}$ activation and IFN$\beta$ mRNA expression, subsequently inhibiting NO production by iNOS. Despite the important role of NO in physiological status, its overproduction by iNOS is involved in several pathologic inflammatory conditions (Ando et al., 2005).

Likewise, cardamonin, another flavonoid from A. galanga, decreased the cardiotoxicity of doxorubicin (DOX) via inhibition of both inflammation and oxidative stress through the Nrf2 pathway. Nrf2 has a close cross-talk with NF- $\kappa \mathrm{B}$ and thus, the antioxidant and anti-inflammatory effect of galangal via these pathways is further confirmed (Qi et al., 2020).

Galangin exhibited nephroprotective effects in the highfructose diet-induced renal damage by inhibition of oxidative damage (Sivakumar et al., 2010).

Taken together, galangal species seem to have direct antiviral properties, as well as protective effects on the main organs damaged in SARS-CoV-2 infection and may be suitable complementary therapies in this infection.

\section{Garlic (Allium sativum L.)}

Even though garlic, from the family Amaryllidaceae, is one of the most ancient medicinal plants there is always has something new to say about it medicinally. In TPM, it is useful for the primary and secondary prevention of different infections and is recommended that it be used during epidemic infectious diseases. Moreover, it has been proposed as a blood thinner and used for the management of several types of cardiovascular events (Aghili Khorasani, 1771). Garlic owes several of its significant pharmacological activities to the organosulfur compounds which are also responsible for the strong flavor and fragrance of the plant (Li et al., 2013).

A recently published molecular docking analysis demonstrated the high inhibitory effects of garlic volatile organosulfur compounds on the invasion of SARS-CoV-2. This effect was mediated through the inhibition of ACE2, a participant in SARS-CoV-2 infection. Allyl disulfide and allyl trisulfide, the major components of garlic essential oil, showed the highest antiviral activity (Thuy et al., 2020). Garlic has demonstrated therapeutic activity against the Dengue virus, a member of the Flaviviridae family causing a lethal hemorrhagic fever. Diallyl disulfide, diallyl sulfide, and alliin could decrease the inflammatory markers in infected cells through the inhibition 
of oxidative damage (Hall et al., 2017). There are several other reports on the antiviral effects of garlic against influenza viruses $\mathrm{A}$ and $\mathrm{B}$, rhinovirus, rotavirus, HIV, and viral pneumonia. Most of these investigations date back to more than twenty years ago when current, more precise techniques were not available (Bayan et al., 2014). Thus, the results of these antiviral assessments need to be reconfirmed with newly developed laboratory methods.

Garlic aqueous extract could effectively ameliorate pulmonary interstitial alveolitis and macroabscesses in lung damage (Mohi El-Din et al., 2014). In LPS-induced acute pulmonary inflammation, oral administration of S-allylmercaptocysteine could dose-dependently inhibit lung damage. The effect of 60 $\mathrm{mg} / \mathrm{kg}$ of S-allylmercaptocysteine was equal to or higher than 500 $\mathrm{mg} / \mathrm{kg}$ of $\mathrm{N}$-acetylcysteine (positive control), showing a high potency. This compound could suppress pro-inflammatory cytokines, reducing macrophage and neutrophils infiltration into BALF, inhibiting NF- $\mathrm{KB}$ activation, and improving endogenous enzymatic and non-enzymatic antioxidants. Furthermore, Nrf2 and its downstream signals, HO-1 and NAD(P)H: quinone oxidoreductase 1 (NQO1), a cytoprotective mediator in oxidative damage, were increased by $\mathrm{S}$ allylmercaptocysteine (Mo et al., 2020). The effect of garlic essential oil and organosulfur components on human neutrophils was also demonstrated in vitro, where they could improve neutrophils function as an immunomodulatory response (Schepetkin et al., 2019). Moreover, S-allyl-L-cysteine has shown inhibitory effects on bleomycin-induced pulmonary inflammation and fibrosis at $10 \mathrm{mg} / \mathrm{kg}$; though, the potency cannot be accurately judged, since no positive control drug was used (Nie et al., 2019). In addition to animal studies, aged garlic extract (garlic soaked in alcohol) has represented beneficial properties in reducing the number and duration of symptoms in subjects with cold/flu. These results also showed an improved proliferation of natural killer (NK) cells and $\gamma \delta$-T lymphocytes in response to pathogen-associated molecular patterns (Nantz et al., 2012).

In another study, black garlic, another popular product prepared through garlic fermentation, was compared with fresh raw garlic regarding its immunostimulatory effects on macrophages. While fresh garlic could significantly improve the phagocytic activity of macrophages and production of cytokines, black garlic showed only negligible effects on these parameters. This significant variation is attributed to the different polysaccharides in the two extracts, specifically fructans which are degraded in black garlic (Li et al., 2017).

Garlic is also widely used for different cardiologic problems and myocardial protection (Bradley et al., 2016). In this regard, raw garlic homogenate showed protective effects on fructoseinduced oxidative stress in cardiac tissue via the elevation of cardiac $\mathrm{H}_{2} \mathrm{~S}$ and preventing myocardial injury. Additionally, PI3K/AKT signaling, a critical pathway in cell survival linked with Nrf2, was activated by garlic (Padiya et al., 2014). Alliin has demonstrated modulatory effects on autophagy, a mechanism involved in cytoprotection and apoptosis of different cells, including myocardium (Zhao et al., 2019). Similarly, allicin has exhibited anti-inflammatory and antioxidant properties in DOX- induced cardiotoxicity (Abdel-Daim et al., 2017). Allicin is produced from alliin by alliinase after chopping garlic and is further metabolized into diallyl trisulfide. The latter compound has exerted cardioprotective effects through AMP-activated protein kinase (AMPK), GSK-3 $\beta$, and hypoxia-inducible factors (HIF)-1 $\alpha$ (Yu et al., 2017). Considering the doses of alliin, allicin, diallyl trisulfide $(20,100 \mathrm{mg} / \mathrm{kg})$ versus garlic homogenate $(250 \mathrm{mg} / \mathrm{kg})$, it can be hypothesized that the purified compounds are more potent than garlic. However, a study on all of these components in the same setting is required to clarify this hypothesis.

Diallyl trisulfide has also exhibited nephroprotective activity in arsenic-induced kidney damage. In addition to the improvement of BUN and creatinine as routine markers of renal function, the compound could modify several markers of inflammation, apoptosis, and oxidative stress (Table 2) (Miltonprabu et al., 2017). A similar activity was observed with in vitro and in vivo administration of S-allylmercaptocysteine in cisplatin-induced kidney injury. Furthermore, p-53, an initiator of apoptosis, and its downstream proteins $\mathrm{B}$-cell associated $\mathrm{X}$ protein (Bax) and $\mathrm{Bcl}-2$ were regulated by this compound. Poly ADP ribose polymerase (PARP), a DNA repairing enzyme during apoptosis which is also under regulation of p-53, was decreased as well, revealing the reversal of cisplatin-induced DNA damage (Zhu et al., 2017).

In general, garlic and its organosulfur compounds act as a multitargeted therapy in several tissues susceptible to SARSCoV-2 injury and may be beneficial as a primary/secondary prevention in these patients.

\section{Grape and Raisin (Vitis vinifera L.)}

Grape, from the family Vitaceae, is one of the most respected fruits in TPM since it produces a series of physiologically balanced humors and thus, is recommended for the general population. In addition to its high nutritional value, modern pharmacological investigations have focused on the remarkable antioxidant properties of this fruit. This attention is due to polyphenolic compounds including proanthocyanidins in the flesh and resveratrol in the fruit seed/peel (Nassiri-Asl and Hosseinzadeh, 2016). Intriguingly, it is emphasized in TPM to take grape with its seed for the urologic problems, showing the knowledge of Persian physicians about the specific effects of the seeds.

In a virtual screening considering influenza A virus vRNA promoter, as well as an in vitro evaluation, procyanidin, a major component of grape extract, revealed significant antiviral activity (Dai et al., 2012). Grape seed extract has shown inhibitory effects on HCV replication via suppression of virus-induced cyclooxygenase (COX)-2 overexpression. It has also shown a synergistic effect by co-administration with conventional antiHCV medicines including telaprevir, daclatasvir, sofosbuvir (Chen et al., 2016). Suppression of the MAPK/JNK pathway is also involved in the antiviral effect of grape seed extract against both HCV (Chen et al., 2016) and RSV (Lee et al., 2017). Moreover, the extract has prevented ILs, MAPK/JNK, and NF$\kappa \mathrm{B}$ pro-inflammatory cascades and regulated mucin production 
via reducing the expression of several mucin MUC genes in the airway epithelium. Interestingly, this effect is in line with the lung protecting properties of grape as described in TPM, by cleaning the pathological viscous pulmonary mucosa (Lee et al., 2017; Kim et al., 2019).

In lead and arsenic-induced lung inflammation, the extract reduced pulmonary levels of pro-inflammatory cytokines, oxidative damage, and pro-apoptotic markers (Lu et al., 2018; $\mathrm{Hu}$ et al., 2019). In carrageenan-induced acute lung inflammation, a single oral dose of grape seed extract could significantly balance the level of several pro-inflammatory and anti-inflammatory cytokines. In addition, it modulated tumor necrosis factor receptor, a co-activator of effector T lymphocytes, upregulated during lung inflammation (Ahmad et al., 2014). In pulmonary fibrosis induced by bleomycin, the extract prevented leukocytes infiltration and markers of fibrosis including matrix metalloproteinase and collagen deposition and increased the anti-fibrotic marker E-cadherin. The potency of the higher extract dose, $100 \mathrm{mg} / \mathrm{kg}$, was equal to $0.5 \mathrm{mg} / \mathrm{kg}$ of dexamethasone in most parameters (Liu et al., 2017).

The immunomodulatory effect of grape was assessed in the subchronic immunotoxicity by aflatoxin B1, a fungal toxin causing oxidative stress and subsequent cellular damages. Grape seed extract at the dose of $100 \mathrm{mg} / \mathrm{kg}$ showed a significant improvement of endogenous antioxidants and decreased pro-inflammatory cytokines to a similar level to those of the healthy animals (Long et al., 2016).

The cardioprotective effect of the extract was also demonstrated in DOX-induced cardiotoxicity, evident from the modulation of electrocardiogram (ECG) and an improvement of endogenous antioxidants activity (Ammar el et al., 2013). Another specific cardioprotective mechanism that has been reported for grape proanthocyanidins is the modification of protein kinase RNA-like ER kinase (PERK) and eukaryotic translation initiation factor-2 (eIF2 $\alpha)$. In endoplasmic reticulum oxidative stress conditions, overactivation of PERK/ eIF2 $\alpha$ stimulates $\mathrm{C} / \mathrm{EBP}$-homologous protein (CHOP) and subsequently, apoptosis. Grape seed extract could reverse the I/ $\mathrm{R}$-induced upregulation of these pathways and prevent the apoptosis of cardiomyocytes (Wang X. et al., 2017).

The inhibition of endoplasmic reticulum-dependent apoptosis was also involved in the nephroprotective effects of grape in diabetic nephropathy (Gao et al., 2018). In another study in diabetic nephropathy, procyanidin B2 could exhibit antioxidant activity via the prevention of mitochondrial dysfunction, a phenomenon accompanied by increased production of ROS as the byproduct of cell metabolism (Bao et al., 2015). Grape seed extract has represented protective effects against kidney damage via the reduction of caspase enzymes, an important category of participants in apoptosis, as well as inhibition of endothelial and inducible forms of NOS. The potency of $100 \mathrm{mg} / \mathrm{kg}$ of the grape extract was equal or higher than the same dose of $\mathrm{N}$-acetylcysteine as the positive control (Ulusoy et al., 2014). The extract has also shown antiinflammatory properties against arsenic-induced nephrotoxicity through the regulation of pro-inflammatory and anti- inflammatory cytokines, as well as deactivation of NF- $\mathrm{BB}$ (Wang C. et al., 2017).

Considering the above-mentioned beneficial effects of grape, in addition to its nutritional value and safety, we suggest that this fruit to be part of the diet of patients with COVID-19. Since the fresh fruit, having several minerals and natural vitamins, is easily available in almost all parts of the world, the juice can be taken orally or even administered via nasogastric tube.

\section{Jujube (Ziziphus jujuba Mill.)}

Jujube fruit from the family Rhamnaceae is one of the valuable, yet not well-recognized medicinal foods mostly grown in Iran and China (Shahrajabian et al., 2019). It is a popular plant as a traditional remedy for different types of coughs. In TPM, it is mostly considered as a modulator of the quality of humor, mainly by preventing the negative effect of excess heat. It is also described as having soothing effects on pulmonary inflammations and shortness of breath (Aghili Khorasani, 1771). In addition to its high nutritive value, jujube is a rich source of bioactive secondary metabolites such as polyphenols, polysaccharides, and terpenoids (Gao et al., 2013).

Betulinic acid, a triterpene constituent of jujube fruit, has shown antiviral activity against influenza $\mathrm{A}$ virus both in vitro and in vivo. At the concentration of $50 \mu \mathrm{M}$, betulinic acid showed a $98 \%$ inhibition of virus cytopathic effects; while it was not toxic for the host cells. Despite the remission of symptoms in the infected animals, no significant change was observed in viral replication and pro-inflammatory cytokines (except for IFN $\gamma$ ). Thus, further mechanistic investigations are needed to establish the antiviral properties of this compound (Hong et al., 2015).

Acidic polysaccharides are another category of active phytochemicals of jujube fruit with immunostimulatory properties. These effects were evident from the increased indices of main lymphatic organs, i.e. spleen and thymus in animals, showing an improved proliferation of immune cells. Additionally, these polysaccharides contain a series of metal ions which possibly participate in biological activities (Zou et al., 2018).

Two categories of polyphenols of jujube peel, free phenols, and bond phenols, were assessed regarding their cardioprotective activity in ISO-induced heart injury in rats. Both types of phenols demonstrated prophylactic antioxidant properties and modulatory effects on different cardiac ion channels with no significant difference between the two polyphenol categories (Cheng et al., 2012). Jujuboside A, another major triterpenoid of this plant, has represented in vitro cardioprotection via regulating of the PI3K/ AKT/mTOR autophagic pathway (Han et al., 2016).

Despite the valuable properties of the jujube plant, the number of high-quality studies on the different medicinal aspects of this plant are limited. Further investigations regarding the active components of the plant and their mechanisms of action are necessary.

\section{Licorice (Glycyrrhiza glabra L., G. uralensis Fisch.)}

Licorice from the family Leguminosae is a valuable medicinal plant in TPM, as well as several other doctrines of traditional 
medicines. The sweet taste of the roots is mostly due to the triterpenoid glycyrrhizin and the plant has several valuable secondary metabolites such as saponins and flavonoids (Pastorino et al., 2018). In TPM, licorice is highly respected as an antitussive medicine for different types of coughs and lung diseases and is also recommended for chronic fevers due to infections (Aghili Khorasani, 1771).

Licorice is a well-studied plant in terms of its antiviral activity (Wang et al., 2015). In addition to its remarkable antiviral properties against some RNA-viruses like HCV and HIV (Adianti et al., 2014; Fukuchi et al., 2016), licorice has demonstrated remarkable antiviral effects on respiratory viruses. Glycyrrhizin has shown a high inhibitory effect on the in vitro replication of two clinical isolates of SARS (Cinatl et al., 2003). Accordingly, fifteen semisynthetic derivatives of this compound were virtually screened against the virus. Glycoside, amide, and carboxyl moieties can significantly increase the activity in comparison to the original backbone; however, the cytotoxicity was also increased in these new structures (Hoever et al., 2005). In influenza A H5N1-infected lung cells, glycyrrhizin inhibited both viral replication and host cell inflammatory and apoptotic response to the infection, a condition responsible for severe flu symptoms (Michaelis et al., 2011). In silico analysis has demonstrated the ability of twelve licorice components to inhibit influenza neuraminidase (NA), a viral surface enzyme involved in the release of replicated viruses from infected host cells (Grienke et al., 2014). Aqueous licorice extract, glycyrrhizin, and one of its metabolites, $18 \beta$ glycyrrhetinic acid were investigated against human RSV. While the extract and $18 \beta$-glycyrrhetinic acid revealed significant antiviral activity, glycyrrhizin was inactive (Feng Yeh et al., 2013). This observation forms the hypothesis that glycyrrhizin acts as a pro-drug which turns into active metabolites such as $18 \beta$-glycyrrhetinic acid but future investigations are required to examine this idea.

Licorice has been traditionally used for cough in the form of a medicinal candy or lozenge. In a clinical study, patients who underwent thoracic surgery and post-operative double-lumen endotracheal intubation gargled a liquid licorice preparation as a prophylaxis for post-extubation coughing. The incidence of cough and sore throat was significantly lower with licorice compared with placebo (simple sugar syrup) (Ruetzler et al., 2013). In ovalbumin-induced lung inflammation, glycyrrhizic acid caused a dose-dependent regulation of cytokine production by types 1 and 2 of helper T lymphocytes, and the effect of the highest dose (40 mg/kg) was equal to $2 \mathrm{mg} / \mathrm{kg}$ of dexamethasone in several parameters (Ma et al., 2013). In LPS-induced inflammation in murine macrophages, glycyrrhizic acid increased autophagy markers such as LC3-II/I and Beclin-1 via $\mathrm{PI} 3 \mathrm{~K} / \mathrm{AKT} / \mathrm{mTOR}$ pathway. Likewise, the compound demonstrated in vivo protective effects on LPS-induced pulmonary inflammation through the reduction of proinflammatory cytokines. It also decreased the high mobility group box (HMGB)-1, a product of damaged cells further activating pro-inflammatory pathways (Qu et al., 2019). The same mechanism was also reported for glycyrrhizin (Kim et al.,
2015). Moreover, glycyrrhizin represented a stimulatory effect on the nuclear translocation of $\mathrm{Nrf} 2$ and $\mathrm{HO}-1$ expression in macrophages via the p38 MAPK pathway, an important intracellular signaling modulating apoptosis and autophagy in response to pathologic conditions (Kim et al., 2015). Glycyrrhizin could reduce abnormally-increased mucus production both in vitro and in vivo via suppression of MUC5AC mRNA expression, confirming the mucolytic properties of licorice mentioned in TPM. The effect of $45 \mathrm{mg} /$ $\mathrm{kg}$ of glycyrrhizin on goblet cell hyperplasia was nearly equal to 1 $\mathrm{mg} / \mathrm{kg}$ of dexamethasone, while $135 \mathrm{mg} / \mathrm{kg}$ of this compound was higher than dexamethasone (Nishimoto et al., 2010).

G. glabra extracts have shown cardioprotective properties both in vitro and in vivo via the improvement of endogenous antioxidant defense mechanisms (Ojha et al., 2013; Upadhyay et al., 2020). In DOX-induced toxicity in cardiomyocytes, licorice aqueous extract has improved sirtuin (SIRT)-1, a cardioprotective transcription factor, and its downstream proteins, peroxisomes proliferator-activated receptors (PPAR)$\alpha / \gamma$ (Upadhyay et al., 2020). Glycyrrhizic acid has demonstrated an inhibitory effect on the long-lasting (L)-type calcium channels of cardiomyocytes. Although this compound could prevent calcium overload in ISO-induced cardiotoxicity, its potency was lower than verapamil even at the highest $(20 \mathrm{mg} / \mathrm{kg})$ dose (Li et al., 2019). Both hydroethanolic licorice extract and glycyrrhizic acid decreased the release of $\mathrm{CK}-\mathrm{MB}$ and $\mathrm{LDH}$, two indicators of myocardial damage (Ojha et al., 2013; Li et al., 2019).

Glycyrrhizic acid has also exhibited a nephroprotective effect on the LPS-induced renal inflammation via modulation of several apoptosis markers such as Bax, Bcl-2, and caspase-3, as well as the pro-inflammatory cytokines and iNOS activity. The reduction of COX-2 activity and its product, prostaglandin E2 (PGE2), was also involved in the nephroprotective activity of this compound (Zhao et al., 2016).

From the above-mentioned studies, it can be inferred that licorice is a multipotential medicinal plant with both direct antiviral properties and protective effects on vulnerable organs in SARS-CoV-2 infection and thus, can be further investigated as an adjuvant therapy in this disease.

\section{Rhubarb (Rheum palmatum L., R. officinale Baill.)}

Several species of the genus Rheum from the family Polygonaceae are commonly consumed as rhubarb. More than the purgative effects that make it a suitable detoxifying agent, rhubarb has pulmonary tonifying activity from the view of TPM. It helps the body to excrete abnormal (pathologic) phlegm and consequently, reduces the organ's susceptibility to infections (Aghili Khorasani, 1771; Zheng et al., 2013).

R. palmatum extract has demonstrated antiviral activity against CVB3, the main reason for viral myocarditis. Interestingly, the viral titers in the internal organs of infected animals treated with $0.3 \mathrm{~g} / \mathrm{kg}$ of the extract were lower than those that received $0.01 \mathrm{~g} / \mathrm{kg}$ ribavirin as the gold standard. Serum viral RNA was not detected on the last day of the experiment in the 
extract-treated group; while it remained positive in the ribavirin group (Xiong et al., 2012). Rheum emodin, an anthraquinone of rhubarb, has also shown antiviral activity against EV-71, mostly through the suppression of viral maturation and virulence and to a lower extent, viral genome levels and protein expression (Zhong et al., 2017). Sennosides A and B, two other anthraquinones of rhubarb, as well as the extracts of two rhubarb species, $R$. palmatum and $R$. officinale, were assessed against HIV infection. The compounds were assessed regarding their inhibitory effects on three main HIV-1 enzymes, i.e. reverse transcriptase (RT)-associated DNA polymerase (RDDP), integrase, and ribonuclease $\mathrm{H}$ (RNase $\mathrm{H})$. R. officinale extract and sennoside A showed a higher potency toward the inhibition of viral RNase $\mathrm{H}$. Additionally, sennoside $\mathrm{A}$ had a lower $\mathrm{IC}_{50}$ for RDDP and integrase, showing a higher activity against viral replication which was also confirmed in the cell-based assay (Esposito et al., 2016).

Rhein, another anthraquinone structure of rhubarb, has shown protective effects on RSV-induced lung infection via modulation of the host inflammatory response, evident from the reduced levels of pro-inflammatory cytokines and NLRP3. NLRP3 inflammasome is a downstream protein complex of the $\mathrm{NF}-\kappa \mathrm{B}$ pathway, activating caspase-1 and consequently, apoptosis. It should be mentioned that rhein at a dose of 120 $\mathrm{mg} / \mathrm{kg}$ showed similar efficacy to $46 \mathrm{mg} / \mathrm{kg}$ of ribavirin (Shen et al., 2019). In both in vitro and in vivo models of pulmonary fibrosis, emodin caused a decrease in $\alpha$-smooth muscle actin and collagen production. Furthermore, the compound reduced TGF$\beta 1$-dependent phosphorylated Smad2/3, a pro-fibrotic mediator inhibiting fibroblast differentiation to myofibroblasts (Guan et al., 2016). Signal transducer and activator of transcription (STAT)-3, another TGF- $\beta 1$-dependent stimulator of fibroblast activation, as well as HSP-47, a collagen-specific heat shock protein and an indicator of pulmonary fibrosis, were also decreased by emodin, further showing its antifibrotic activity (Guan et al., 2016). Chrysophanol, another anthraquinone from rhubarb, has proved anti-inflammatory properties mostly via the inhibition of NF- $\kappa \mathrm{B}$ activity and the prevention of lung fibrosis. Moreover, this compound could reduce ovalbumin-induced autophagy and inflammation (Song et al., 2019). In a randomized, controlled clinical trial in patients with acute respiratory distress syndrome (ARDS), a life-threatening condition also observed in COVID-19, a liquid rhubarb preparation was administered via nasogastric tube for one week. In comparison to the control group, the adjuvant rhubarb administration could significantly improve oxygenation. Additionally, it could decrease the extravascular lung water index and pulmonary vascular permeability index (He et al., 2017), confirming the results of the preclinical studies in a clinical setting. It should be noted that no identification process was considered for rhubarb and thus, it is not clear which species of the plant were used in this trial (He et al., 2017).

Rhubarb anthraquinones have also demonstrated cardioprotective properties. Emodin could increase the secretion of atrial natriuretic peptide (ANP), a molecule secreted by cardiomyocytes with a multitargeted role in cardioprotection (Zhou et al., 2014). Rhein could restore the downregulation of the PI3K/GSK3 $\beta$ cardioprotective pathway in I/R-induced cardiotoxicity (Liu et al., 2018). Chrysophanol has shown an inhibitory effect on PARylation, the process of PARP attachment to its target proteins, overactivation of which participates in DOX-induced cardiotoxicity. In addition, apoptotic markers, as well as mitochondrial damage were reduced by chrysophanol both in vitro and in vivo (Lu et al., 2019).

Rhubarb extract could inhibit adenine-induced renal damage via the suppression of the TGF- $\beta /$ Smad pathway (Zhang et al., 2018), a mechanism also reported for its protective effects on the lung (Guan et al., 2016). Furthermore, several other fibrosis biomarkers including E-cadherin, collagen, $\alpha$-smooth muscle actin and vimentin were decreased in rats treated with rhubarb (Zhang et al., 2018). The aqueous $R$. palmatum extract and rhein have demonstrated nephroprotective activity in cellular and animal models of chronic kidney disease. This effect was mediated via suppression of autophagy-related pathways and renal fibrosis (Tu et al., 2017).

The result of preclinical studies on rhubarb show the pharmacological activity of this plant to be highly attributed to its anthraquinones through several therapeutic targets in organs damaged in SARS-CoV-2 infection. Especially, a clinical study of the protective effects of this plant in patients with ARDS represents the significant efficacy and acceptable safety of its use in treating a condition remarkably similar to COVID-19. Thus, rhubarb could be one of the possible choices that are clinically assessed in treating this disease.

\section{Saffron (Crocus sativus L.)}

Saffron, known as the red gold, is the stigma of an herbaceous plant from the family Iridaceae, native to Iran, with several pharmacological activities. The most investigated components of the plant are carotenoid structures including crocins and their metabolites, crocetin responsible for the saffron color. The terpene glycoside picrocrocin, as well as the monoterpene aldehyde safranal, a volatile compound causing the specific saffron aroma are other important components of saffron (Boskabady and Farkhondeh, 2016). In TPM, Saffron is considered to be a tonic of the lung and kidneys. It is highly valued not only as a cardiotonic medicine, but also as a means of enhancing the delivery of other medicinal ingredients to the heart. Thus, saffron is one of the most frequently used ingredients in TPM multicomponent preparations for heart diseases (Aghili Khorasani, 1771; Sadati et al., 2016).

The aqueous extract of saffron, as well as crocin and picrocrocin, were evaluated in terms of in vitro antiviral activity against HIV-1. Both carotenoids showed antiviral activity with a relatively low $\mathrm{IC}_{50}(5$ and $8 \mu \mathrm{M})$ and high SI (>187 and 600)(Table 2), showing these compounds to be potent antiviral agents. On the other hand, the aqueous extract showed no considerable antiviral activity, revealing that the antiviral activity of the plant is mostly due to its lipophilic compounds such as carotenoids (Soleymani et al., 2018).

Safranal has demonstrated anti-inflammatory effects on OVA-induced airway inflammation via modulation of type 1 
and 2 helper $\mathrm{T}$ lymphocytes balance, evident from the reduced serum IL-4 and elevated IFN $\gamma$ (Boskabady et al., 2014). This modulatory effect on cytokine production was also observed in vitro in T lymphocytes (Boskabady et al., 2011). Interestingly, even the effect of the lowest dose ( $4 \mu \mathrm{g} / \mathrm{ml}$ of drinking water) was higher than $50 \mu \mathrm{g} / \mathrm{ml}$ of dexamethasone, showing a significantly higher potency for this compound in alleviating pulmonary inflammation (Boskabady et al., 2014). In bleomycin-induced pulmonary damage, crocin has reduced tissue inflammation via the reduction of pro-inflammatory cytokines and markers of fibrosis, improvement of endogenous antioxidant mechanisms, and the induction of the Nrf2 cytoprotective pathway. Likewise, the compound was an inhibitor of Toll-like receptor (TLR)-4, a receptor participating in leukocytes infiltration, neutrophils activation, TNF- $\alpha$-dependent inflammation, and TGF- $\beta$ dependent fibrosis. The antifibrotic effect of crocin at $20 \mathrm{mg} / \mathrm{kg}$ was higher than $0.2 \mathrm{mg} / \mathrm{kg}$ of halofuginone (Zaghloul et al., 2019). Additionally, crocin has been shown to relieve the effects of LPS-induced acute lung injury by suppressing NF- $\mathrm{KB}$ and MAPK pro-inflammatory cascades. Matrix metalloproteinase 9 (MMP-9), heparanse, and two glycocalyx shedding enzymes overactivated in inflammatory lung diseases were also decreased by crocin (Zhang et al., 2020b). In a randomized, triple-blind, placebo-controlled clinical trial in patients with mild to moderate asthma, a saffron powder supplement was administered for two months. Spirometry parameters, including forced vital capacity (FVC), forced expiratory volume in the first second (FEV1), FEV1/FVC ratio, and forced expiratory flow 25-75\% were significantly improved in comparison to placebo. Furthermore, serum levels of CRP and the anti-HSP70 antibody were significantly decreased. A direct correlation between the severity of asthma symptoms and anti-HSP70 antibody was observed and thus, its reduction is an indicator of decreased pulmonary inflammation (Hosseini et al., 2018).

Saffron aqueous extract exhibited cardioprotective effects on an animal model of ISO-induced cardiac damage via reduction of $\mathrm{CK}-\mathrm{MB}$ and $\mathrm{LDH}$ leakage from myocardial cells and improvement of endogenous antioxidants in cardiac tissue (Sachdeva et al., 2012). A similar result was obtained with safranal from a significantly lower effective dose in comparison to the aqueous extract, suggesting these effects are partially due to this saffron component (Mehdizadeh et al., 2013). In DOX plus I/ R-induced cardiotoxicity, saffron could restore the level of contractile proteins including $\alpha$-actinine, myosin light chain, and troponine C. It could prevent mitochondrial dysfunction and recover the phosphorylation level of the AKT/P70S6K and ERK1/2 cardioprotective pathways (Chahine et al., 2016). These effects may be partially mediated by crocin since this compound has shown to have protective activity with respect to ECG in an animal model of DOX-induced cardiotoxicity (Razmaraii et al., 2016). Similarly, crocin was effective in the reduction of LPSinduced cardiotoxicity via a decrease in the protein level and gene expression of pro-inflammatory cytokines. COX-2 enzyme and its product, PGE2, which are increased in LPS-induced damages were returned to the normal level by crocin administration (Rahim et al., 2019).
Saffron extract has shown detoxifying effects on alcoholinduced renal damage mostly through the inhibition of proapoptotic mediators including caspase enzymes and $\mathrm{Bax} / \mathrm{Bcl}-2$ signaling, as well as pro-inflammatory cytokines production (Rezaee-Khorasany et al., 2019). Crocin has demonstrated nephroprotective properties in an animal model of diabetesinduced nephrotoxicity (Table 2). Aside from general antioxidant properties (Erdemli et al., 2017), this compound could decrease hyperurocemia via the inhibition of xanthine oxidase (Altinoz et al., 2015). Moreover, a clinical trial using 100 $\mathrm{mg} /$ day of saffron in healthy subjects showed this plant to have a short-term immunopotentiation, considering the altered levels of immunoglobulins and the leukocytes count (Kianbakht and Ghazavi, 2011).

\section{DISCUSSION AND CONCLUSIONS}

This paper has reviewed the pharmacological mechanisms of medicinal plants with possible beneficial effects on SARS-CoV-2 infection and related organ damage based on the suggestions of TPM. Most of the medicinal plants included in this review show multitargeted activity and protective mechanisms in the tissues damaged in SARS-CoV-2 infection.

The most important effects of the medicinal plants and their isolated phytochemicals include anti-inflammatory activity and antioxidant properties (Figure 1). These two mechanisms comprise several cellular and subcellular pathways such as NF$\kappa \mathrm{B}$, Nrf2, pro-inflammatory and anti-inflammatory cytokines balance, and endogenous enzymatic/non-enzymatic antioxidant defense mechanisms. It should be considered that such mechanisms are general cytoprotective cascades observed in nearly all body organs and that the beneficial effects of phytochemicals in one tissue can be extrapolated to other tissues. For instance, if a compound has shown a stimulatory effect on Nrf2 signaling in myocardial cells, the same activity could be expected in lung tissue or kidney tubules. This is in line with the holistic view of TPM, which encompasses the idea that treatments should improve the overall health of the human body as a whole, instead of focusing on the damaged organ. It is believed in TPM that reinforcement of the body's inner power is one of the best ways to combat a disease. Most of the discussed medicinal plants stimulate cytoprotective mechanisms to face pathogenic factors, which can be considered as equivalent to these ideas of improving the body's inner power.

One of the key pro-inflammatory cytokines affecting clinical manifestations of COVID-19 is IL-6. In patients with COVID19 , organ failures have been observed and the subsequent deaths of many patients are often due to cytokine storm, an exaggerated inflammatory response (Mehta et al., 2020). IL-6 has a major role in this event and the IL-6 inhibitor tociluzumab (Actemra ${ }^{\circledR}$ ) has been evaluated in several clinical studies in patients. Another systematic review has also discussed the value of this agent in treating COVID-19, highlighting that current evidence suggests that the drug could be beneficial in this infection (Alzghari and Acuña, 2020). Another important cytokine in this infection is 


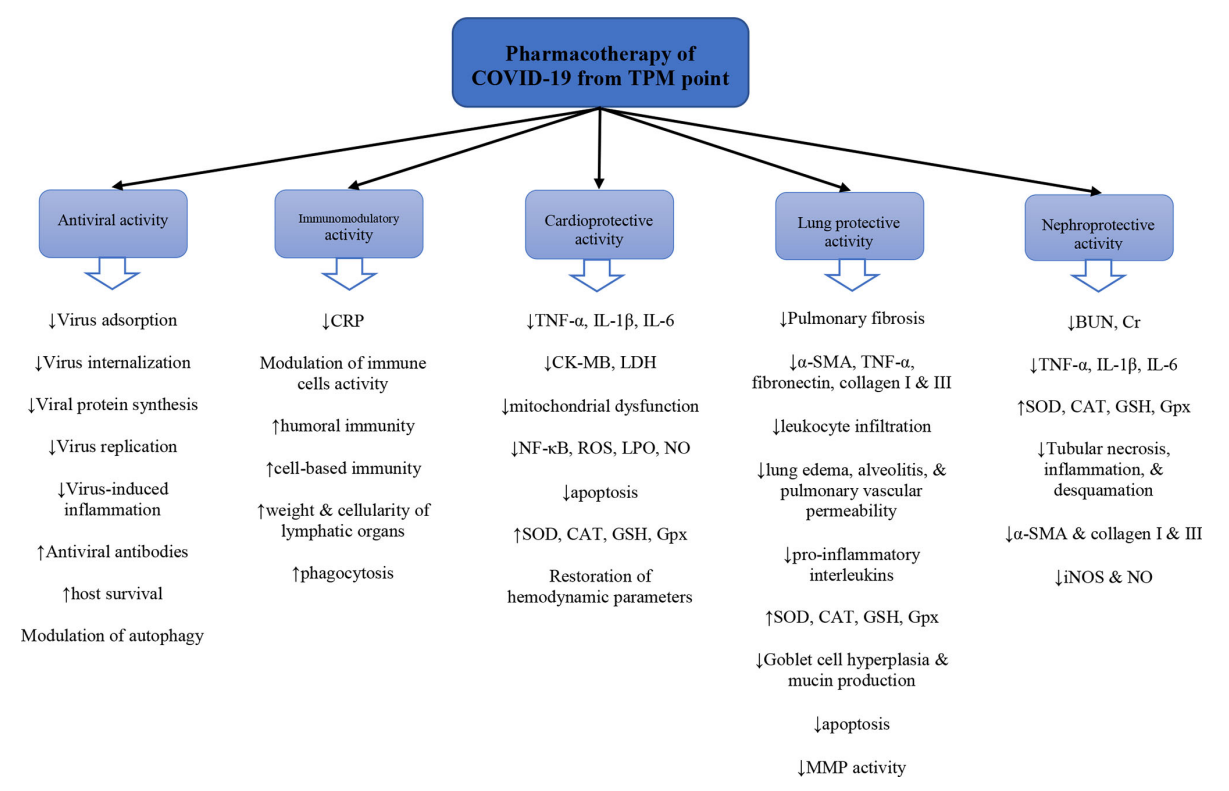

FIGURE 1 | Mechanisms of medicinal plants introduced based on traditional Persian medicine for the management of SARS-CoV-2 infection.

TNF- $\alpha$ and the inhibitors of this cytokine are also currently being assessed as therapeutic options for SARS-CoV-2 patients (Feldmann et al., 2020). A considerable number of the plants discussed in this present review have been shown to reduce IL-6 and TNF- $\alpha$ levels and/or activities (Table 2). Major phytochemicals of these plants should be further investigated in the design and development of plant-derived cytokine inhibitors in future studies.

Edible plants, particularly grape, jujube fruit, amla, and damask rose, are well-known and used medicinally every day by people all over the world, which indicates that they are safe. Such plants can be prepared as a juice or in a standardized liquid dosage form for COVID-19 patients. Where the guidelines on effective drugs for treating this infection vary day-by-day, increasing amounts of new data are being released from ongoing clinical trials regarding the pros and cons of the medicines recommended. In such a situation, physicians may be willing to stay on the safe side and avoid using multiingredient complementary medicinal preparations with several possible side effects/drug interactions. As a more acceptable and safer approach, natural tonics that are based on these popular food plants could be recommended as part of the healthy daily diet for people prone to or who are vulnerable to the disease. This includes healthcare providers caring for COVID-19 patients in hospitals, the family members of the patients in homequarantine, and people with underlying diseases such as cardiovascular problems or diabetes who are more vulnerable to this infection. Studies on healthy populations are encouraged due to the lower risk of adverse effects and the fact that they would indicate the safety and efficacy of natural products, with use in patients as the second step in developing these adjunct therapies.
On the other hand, some phytochemicals have revealed direct antiviral activity via blockade of different stages of the virus life cycle including fusion, replication, protein synthesis, and viral particle release from the host cells. Although the life cycle and target proteins of SARS-CoV-2 may differ from the assessed viruses to some extent, there would be similarities that are worth assessing in terms of the antiviral activity of these compounds against the virus. As has been reported in virtual screenings (Zhang et al., 2020a), some of these compounds have shown significant interactions with SARS-CoV-2 structures, further confirming their antiviral activity. In this regard, molecular docking analyses would help in selecting structures with the highest binding affinity and the ability to inhibit viral enzymes. The virtually selected antiviral phytochemicals can then be evaluated in cell-based and animal studies. Likewise, they can be considered as molecular backbones in the design of new semisynthetic structures and in creating new compounds with higher safety and antiviral efficacy.

Some of the included compounds such as quercetin, pyrogallol, and fructan polysaccharides are considered ubiquitous, i.e. they can be found in several foods and spices of the human diet and do not belong to a specific medicinal plant. Despite the abundance of these compounds, their pharmacological activity cannot be denied and further interpretation of the results of studies is of great importance. For example, in an in vitro study by Mahmood et al. (1996) quercetin showed the highest antiviral activity against HIV amongst several evaluated phytochemicals. This does not mean that any quercetin-containing plant can be effective against viral infection. Instead, the effective dose of this compound should be measured in preclinical studies and a dose translation calculation is needed to suggest accurate human doses of quercetin and to observe related pharmacological activity. 
An important limitation of the studies included in this review is that many of them lacked a positive control group. Some of the reports used dexamethasone as a standard anti-inflammatory agent in antiviral evaluations, however, most of the studies did not consider a standard treatment. This methodological problem makes any judgment regarding the potency of the assessed materials difficult. Some antiviral phytochemicals have significantly lower SI compared with conventional antiviral agents which excludes them from further assessment. Lack of a positive control makes such comparisons difficult since SI reported in other studies do not provide an accurate comparison due to these methodological differences. The same problem exists in the evaluation of the other pharmacological properties of the plants. Thus, future studies should consider the use of a gold standard drug for a better presentation of the potency of test agents.

One of the concerns in the clinical use of medicinal plants is the possibility of herb-drug interaction. Phytochemicals can have inducing (Soleymani et al., 2017) or inhibitory effects (Bahramsoltani et al., 2017) on drug metabolizing enzymes such as hepatic cytochrome P450 and intestinal P-glycoprotein and thus, can affect the pharmacokinetics of conventional drugs. Consequently, the serum level of conventional antiviral agents administered to COVID-19 patients may be affected by concomitant administration of herbal supplements. Such pharmacokinetic interactions may increase serum level and adverse effects, or decrease serum concentration and clinical response, both of which can have life-threatening outcomes in patients.

Another discussion can be raised concerning the plants that contain ACE inhibitor compounds. ACE inhibitors do not have a direct effect on SARS-CoV-2 but they can increase the expression of the ACE2 receptors. This receptor acts as a co-receptor in the invasion of the virus and thus, a hypothesis is formed that ACE inhibitors may increase susceptibility to SARS-CoV-2 (Danser et al., 2020).

Most of the studies included in this review were animal and cell-based evaluations, providing only foundational evidence for the efficacy of the aforementioned plants in human studies. Only six clinical studies were considered in this paper and higher levels of evidence are essential to examine the medicinal suggestions of TPM in clinical settings. Although there are several ongoing clinical trials on the effect of TPM formulas on COVID-19, no

\section{REFERENCES}

Abdel-Daim, M. M., Kilany, O. E., Khalifa, H. A., and Ahmed, A. A. M. (2017). Allicin ameliorates doxorubicin-induced cardiotoxicity in rats via suppression of oxidative stress, inflammation and apoptosis. Cancer Chemother. Pharmacol. 80 (4), 745-753. doi: 10.1007/s00280-017-3413-7

Abubakar, I. B., Malami, I., Yahaya, Y., and Sule, S. M. (2018). A review on the ethnomedicinal uses, phytochemistry and pharmacology of Alpinia officinarum Hance. J. Ethnopharmacol. 224, 45-62. doi: 10.1016/ j.jep.2018.05.027

Adianti, M., Aoki, C., Komoto, M., Deng, L., Shoji, I., Wahyuni, T. S., et al. (2014). Anti-hepatitis C virus compounds obtained from Glycyrrhiza uralensis and published data is available. Despite the challenges in decisionmaking for the clinical assessment of natural products in SARSCoV-2, high-quality trials have evaluated the effect of such products with valuable outcomes (Yang et al., 2020), with over fifty clinical trials designed to assess the effect of self-made and commercial TCM formulas. The National Health Commission of China has also added some TCM recommendations to the latest guidelines for the management of COVID-19 patients, even in severe cases (Yang et al., 2020). Thus, the trial design and inclusion/exclusion criteria for patients, as well as drug preparation can be defined in a way that is comprehensively approved. Natural compounds may have some advantages in comparison to conventional drugs. For instance, there are concerns about the currently available TNF- $\alpha$ inhibitors because they can suppress the immune system, leading to secondary bacterial/viral infections (Feldmann et al., 2020). On the other hand, a remarkable number of natural antiviral compounds have immunomodulatory and antibacterial properties. Further studies in healthy individuals that assess the prophylactic activity of these supplements as well as trials in patients with less severe symptoms would pave the way for further clinical evaluations.

In conclusion, medicinal plants have great potential value and can be recommended for treatment of COVID-19 based on the therapeutic approaches of TPM, several of which have also been confirmed by pharmacological studies in modern medicine. The currently available data, regarding these medicinal plants, provide foundational evidence. Future preclinical mechanistic studies as well as clinical trials are necessary to confirm the safety and efficacy of these plants for the management of SARS-CoV2 infection.

\section{AUTHOR CONTRIBUTIONS}

All authors contributed to the article and approved the submitted version.

\section{FUNDING}

This work is partially supported by the Tehran University of Medical Sciences (Grant No. 99-1-147-47262). other Glycyrrhiza species. Microbiol. Immunol. 58 (3), 180-187. doi: 10.1111/ 1348-0421.12127

Aghili Khorasani, M. H. (1771). Makhzan-al-Adviah (Rewritten by Shams Ardakani MR, Rahimi R, Farjadmand F. 1st ed) (Tehran: Tehran University of Medical Sciences).

Ahmad, S. F., Zoheir, K. M., Abdel-Hamied, H. E., Attia, S. M., Bakheet, S. A., Ashour, A. E., et al. (2014). Grape seed proanthocyanidin extract protects against carrageenan-induced lung inflammation in mice through reduction of pro-inflammatory markers and chemokine expressions. Inflammation 37 (2), 500-511. doi: 10.1007/s10753-013-9764-2

Altinoz, E., Oner, Z., Elbe, H., Cigremis, Y., and Turkoz, Y. (2015). Protective effects of saffron (its active constituent, crocin) on nephropathy in 
streptozotocin-induced diabetic rats. Hum. Exp. Toxicol. 34 (2), 127-134. doi: $10.1177 / 0960327114538989$

Alzghari, S. K., and Acuña, V. S. (2020). Supportive treatment with Tocilizumab for COVID-19: A systematic review. J. Clin. Virol. 127, 104380. doi: 10.1016/ j.jcv.2020.104380

Ammar el, S. M., Said, S. A., El-Damarawy, S. L., and Suddek, G. M. (2013). Cardioprotective effect of grape-seed proanthocyanidins on doxorubicininduced cardiac toxicity in rats. Pharm. Biol. 51 (3), 339-344. doi: 10.3109/ 13880209.2012.729065

Ando, S., Matsuda, H., Morikawa, T., and Yoshikawa, M. (2005). 1'S-1'Acetoxychavicol acetate as a new type inhibitor of interferon-beta production in lipopolysaccharide-activated mouse peritoneal macrophages. Bioorg. Med. Chem. 13 (9), 3289-3294. doi: 10.1016/j.bmc.2005.02.022

Avicenna, (1025). The Canon of Medicine (Qanoon-fil-Tib). Translated by sharafkandi. A. Tehran; 1991: Soroush, Vol. 2.

Bachiega, T. F., de Sousa, J. P., Bastos, J. K., and Sforcin, J. M. (2012). Clove and eugenol in noncytotoxic concentrations exert immunomodulatory/antiinflammatory action on cytokine production by murine macrophages. J. Pharm. Pharmacol. 64 (4), 610-616. doi: 10.1111/j.2042-7158.2011.01440.x

Bahramsoltani, R., Sodagari, H. R., Farzaei, M. H., Abdolghaffari, A. H., Gooshe, M., and Rezaei, N. (2016). The preventive and therapeutic potential of natural polyphenols on influenza. Expert Rev. Anti Infect. Ther. 14 (1), 57-80. doi: $10.1586 / 14787210.2016 .1120670$

Bahramsoltani, R., Rahimi, R., and Farzaei, M. H. (2017). Pharmacokinetic interactions of curcuminoids with conventional drugs: A review. J. Ethnopharmacol. 209, 1-12. doi: 10.1016/j.jep.2017.07.022

Bao, L., Cai, X., Zhang, Z., and Li, Y. (2015). Grape seed procyanidin B2 ameliorates mitochondrial dysfunction and inhibits apoptosis via the AMPactivated protein kinase-silent mating type information regulation 2 homologue 1-PPARgamma co-activator-1alpha axis in rat mesangial cells under high-dose glucosamine. Br. J. Nutr. 113 (1), 35-44. doi: 10.1017/ s000711451400347x

Bayan, L., Koulivand, P. H., and Gorji, A. (2014). Garlic: a review of potential therapeutic effects. Avicenna J. Phytomed. 4 (1), 1.

Bhattacharya, S. K., Bhattacharya, A., Sairam, K., and Ghosal, S. (2002). Effect of bioactive tannoid principles of Emblica officinalis on ischemia-reperfusioninduced oxidative stress in rat heart. Phytomedicine 9 (2), 171-174. doi: 10.1078/0944-7113-00090

Boskabady, M. H., Kiani, S., and Rakhshandah, H. (2006). Relaxant effects of Rosa damascena on guinea pig tracheal chains and its possible mechanism(s). J. Ethnopharmacol. 106 (3), 377-382. doi: 10.1016/j.jep.2006.01.013

Boskabady, M. H., Seyedhosseini Tamijani, S. M., Rafatpanah, H., Rezaei, A., and Alavinejad, A. (2011). The effect of Crocus sativus extract on human lymphocytes' cytokines and T helper 2/T helper 1 balance. J. Med. Food 14 (12), 1538-1545. doi: 10.1089/jmf.2011.1697

Boskabady, M. H., Vatanprast, A., Parsaee, H., and Boskabady, M. (2013). Possible mechanism of inotropic and chronotropic effects of Rosa damascena on isolated guinea pig heart. Daru 21 (1), 38. doi: 10.1186/2008-2231-21-38

Boskabady, M. H., Byrami, G., and Feizpour, A. (2014). The effect of safranal, a constituent of Crocus sativus (saffron), on tracheal responsiveness, serum levels of cytokines, total $\mathrm{NO}$ and nitrite in sensitized guinea pigs. Pharmacol. Rep. 66 (1), 56-61. doi: 10.1016/j.pharep.2013.08.004

Boskabady, M. H., and Farkhondeh, T. (2016). Antiinflammatory, Antioxidant, and Immunomodulatory Effects of Crocus sativus L. and its Main Constituents. Phytother. Res. 30 (7), 1072-1094. doi: 10.1002/ptr.5622

Bradley, J. M., Organ, C. L., and Lefer, D. J. (2016). Garlic-Derived Organic Polysulfides and Myocardial Protection. J. Nutr. 146 (2), 403s-409s. doi: 10.3945/jn.114.208066

Carrasco, F. R., Schmidt, G., Romero, A. L., Sartoretto, J. L., Caparroz-Assef, S. M., Bersani-Amado, C. A., et al. (2009). Immunomodulatory activity of Zingiber officinale Roscoe, Salvia officinalis L. and Syzygium aromaticum L. essential oils: evidence for humor- and cell-mediated responses. J. Pharm. Pharmacol. 61 (7), 961-967. doi: 10.1211/jpp/61.07.0017

Chahine, N., Nader, M., Duca, L., Martiny, L., and Chahine, R. (2016). Saffron extracts alleviate cardiomyocytes injury induced by doxorubicin and ischemiareperfusion in vitro. Drug Chem. Toxicol. 39 (1), 87-96. doi: 10.3109/ 01480545.2015 .1036281
Chaieb, K., Hajlaoui, H., Zmantar, T., Kahla-Nakbi, A. B., Rouabhia, M., Mahdouani, K., et al. (2007). The chemical composition and biological activity of clove essential oil, Eugenia caryophyllata (Syzigium aromaticum L. Myrtaceae): a short review. Phytother. Res. 21 (6), 501-506. doi: 10.1002/ ptr.2124

Chen, W. C., Tseng, C. K., Chen, B. H., Lin, C. K., and Lee, J. C. (2016). Grape Seed Extract Attenuates Hepatitis C Virus Replication and Virus-Induced Inflammation. Front. Pharmacol. 7, 490. doi: 10.3389/fphar.2016.00490

Chen, Z., and Nakamura, T. (2004). Statistical evidence for the usefulness of Chinese medicine in the treatment of SARS. Phytother. Res. 18 (7), 592-594. doi: $10.1002 /$ ptr.1485

Cheng, D., Zhu, C., Cao, J., and Jiang, W. (2012). The protective effects of polyphenols from jujube peel (Ziziphus Jujube Mill) on isoproterenol-induced myocardial ischemia and aluminum-induced oxidative damage in rats. Food Chem. Toxicol. 50 (5), 1302-1308. doi: 10.1016/j.fct.2012.01.026

Chniguir, A., Zioud, F., Marzaioli, V., El-Benna, J., and Bachoual, R. (2019). Syzygium aromaticum aqueous extract inhibits human neutrophils myeloperoxidase and protects mice from LPS-induced lung inflammation. Pharm. Biol. 57 (1), 56-64. doi: 10.1080/13880209.2018.1557697

Cinatl, J., Morgenstern, B., Bauer, G., Chandra, P., Rabenau, H., and Doerr, H. (2003). Glycyrrhizin, an active component of liquorice roots, and replication of SARS-associated coronavirus. Lancet 361 (9374), 2045-2046. doi: 10.1016/ S0140-6736(03)13615-X

Cui, H. T., Li, Y. T., Guo, L. Y., Liu, X. G., Wang, L. S., Jia, J. W., et al. (2020). Traditional Chinese medicine for treatment of coronavirus disease 2019: a review. Tradit. Med. Res. 5 (2), 65-73. doi: 10.12032/TMR20200222165

Dai, J., Wang, G., Li, W., Zhang, L., Yang, J., Zhao, X., et al. (2012). Highthroughput screening for anti-influenza $A$ virus drugs and study of the mechanism of procyanidin on influenza A virus-induced autophagy. J. Biomol. Screen 17 (5), 605-617. doi: 10.1177/1087057111435236

Dai, J. P., Zhao, X. F., Zeng, J., Wan, Q. Y., Yang, J. C., Li, W. Z., et al. (2013). Drug screening for autophagy inhibitors based on the dissociation of Beclin1-Bcl2 complex using BiFC technique and mechanism of eugenol on anti-influenza $\mathrm{A}$ virus activity. PloS One 8 (4), e61026. doi: 10.1371/journal.pone.0061026

Danser, A. J., Epstein, M., and Batlle, D. (2020). Renin-angiotensin system blockers and the COVID-19 pandemic: at present there is no evidence to abandon renin-angiotensin system blockers. Hypertension 75 (6), 1382-1385. doi: 10.1161/ HYPERTENSIONAHA.120.15082

el-Mekkawy, S., Meselhy, M. R., Kusumoto, I. T., Kadota, S., Hattori, M., and Namba, T. (1995). Inhibitory effects of Egyptian folk medicines on human immunodeficiency virus (HIV) reverse transcriptase. Chem. Pharm. Bull. (Tokyo) 43 (4), 641-648. doi: 10.1248/cpb.43.641

El-Seedi, H. R., Khalifa, S. A., Yosri, N., Khatib, A., Chen, L., Saeed, A., et al. (2019). Plants mentioned in the Islamic Scriptures (Holy Qur'ân and Ahadith): Traditional uses and medicinal importance in contemporary times. J. Ethnopharmacol. 243:112007. doi: 10.1016/j.jep.2019.112007

Emtiazy, M., Keshavarz, M., Khodadoost, M., Kamalinejad, M., Gooshahgir, S. A., Bajestani, H. S., et al. (2012). Relation between body humors and hypercholesterolemia: An Iranian traditional medicine perspective based on the teaching of Avicenna. Iran Red Crescent Med. J. 14 (3), 133-138.

Emtiazy, M., Oveidzadeh, L., Habibi, M., Molaeipour, L., Talei, D., Jafari, Z., et al. (2018). Investigating the effectiveness of the Trigonella foenum-graecum $\mathrm{L}$. (fenugreek) seeds in mild asthma: a randomized controlled trial. Allergy Asthma Clin. Immunol. 14, 19. doi: 10.1186/s13223-018-0238-9

Erdemli, M. E., Gul, M., Altinoz, E., Zayman, E., Aksungur, Z., and Bag, H. G. (2017). The protective role of crocin in tartrazine induced nephrotoxicity in Wistar rats. BioMed. Pharmacother. 96, 930-935. doi: 10.1016/ j.biopha.2017.11.150

Esposito, F., Carli, I., Del Vecchio, C., Xu, L., Corona, A., Grandi, N., et al. (2016). Sennoside A, derived from the traditional chinese medicine plant Rheum L., is a new dual HIV-1 inhibitor effective on HIV-1 replication. Phytomedicine 23 (12), 1383-1391. doi: 10.1016/j.phymed.2016.08.001

Feki, A., Ben Saad, H., Bkhairia, I., Ktari, N., Naifar, M., Boudawara, O., et al. (2019). Cardiotoxicity and myocardial infarction-associated DNA damage induced by thiamethoxam in vitro and in vivo: Protective role of Trigonella foenum-graecum seed-derived polysaccharide. Environ. Toxicol. 34 (3), 271282. doi: $10.1002 /$ tox. 22682 
Feldmann, M., Maini, R. N., Woody, J. N., Holgate, S. T., Winter, G., Rowland, M., et al. (2020). Trials of anti-tumour necrosis factor therapy for COVID-19 are urgently needed. Lancet 395 (10234), 1407-1409. doi: 10.1016/S0140-6736(20) 30858-8

Feng Yeh, C., Wang, K. C., Chiang, L. C., Shieh, D. E., Yen, M. H., and San Chang, J. (2013). Water extract of licorice had anti-viral activity against human respiratory syncytial virus in human respiratory tract cell lines. J. Ethnopharmacol. 148 (2), 466-473. doi: 10.1016/j.jep.2013.04.040

Fukuchi, K., Okudaira, N., Adachi, K., Odai-Ide, R., Watanabe, S., Ohno, H., et al. (2016). Antiviral and Antitumor Activity of Licorice Root Extracts. In Vivo 30 (6), 777-785. doi: 10.21873/invivo.10994

Gao, Q.-H., Wu, C.-S., and Wang, M. (2013). The jujube (Ziziphus jujuba Mill.) fruit: a review of current knowledge of fruit composition and health benefits. J. Agric. Food Chem. 61 (14), 3351-3363. doi: 10.1021/jf4007032

Gao, Z., Liu, G., Hu, Z., Shi, W., Chen, B., Zou, P., et al. (2018). Grape seed proanthocyanidins protect against streptozotocininduced diabetic nephropathy by attenuating endoplasmic reticulum stressinduced apoptosis. Mol. Med. Rep. 18 (2), 1447-1454. doi: 10.3892/mmr.2018.9140

Ghahreman, A., and Okhovvat, A. R. (2009). Matching the old medicinal plant names with scientific terminology Vol. 2 (Tehran, Iran: University of Tehran Publishing Group).

Goraya, R. K., and Bajwa, U. (2015). Enhancing the functional properties and nutritional quality of ice cream with processed amla (Indian gooseberry). J. Food Sci. Technol. 52 (12), 7861-7871. doi: 10.1007/s13197-015-1877-1

Grienke, U., Braun, H., Seidel, N., Kirchmair, J., Richter, M., Krumbholz, A., et al. (2014). Computer-guided approach to access the anti-influenza activity of licorice constituents. J. Nat. Prod. 77 (3), 563-570. doi: 10.1021/np400817j

Guan, R., Zhao, X., Wang, X., Song, N., Guo, Y., Yan, X., et al. (2016). Emodin alleviates bleomycin-induced pulmonary fibrosis in rats. Toxicol. Lett. 262, 161-172. doi: 10.1016/j.toxlet.2016.10.004

Hall, A., Troupin, A., Londono-Renteria, B., and Colpitts, T. M. (2017). Garlic Organosulfur Compounds Reduce Inflammation and Oxidative Stress during Dengue Virus Infection. Viruses 9 (7), 159. doi: 10.3390/v9070159

Hamedi, A., Zarshenas, M. M., Sohrabpour, M., and Zargaran, A. (2013). Herbal medicinal oils in traditional Persian medicine. Pharmaceut. Biol. 51 (9), 12081218. doi: 10.3109/13880209.2013.777462

Han, D., Wan, C., Liu, F., Xu, X., Jiang, L., and Xu, J. (2016). Jujuboside A Protects H9C2 Cells from Isoproterenol-Induced Injury via Activating PI3K/Akt/ mTOR Signaling Pathway. Evid Based Complement. Alternat. Med. 2016, 9593716. doi: 10.1155/2016/9593716

He, J., Si, X., Ji, M., Huang, J., Zheng, W., Wang, J., et al. (2017). Effect of rhubarb on extravascular lung water in patients with acute respiratory distress syndrome. Rev. Assoc. Med. Bras. (1992) 63 (5), 435-440. doi: 10.1590/18069282.63.05.435

Hoever, G., Baltina, L., Michaelis, M., Kondratenko, R., Baltina, L., Tolstikov, G. A., et al. (2005). Antiviral activity of glycyrrhizic acid derivatives against SARScoronavirus. J. Med. Chem. 48 (4), 1256-1259. doi: 10.1021/jm0493008

Hong, E. H., Song, J. H., Kang, K. B., Sung, S. H., Ko, H. J., and Yang, H. (2015). Anti-Influenza Activity of Betulinic Acid from Zizyphus jujuba on Influenza A/ PR/8 Virus. Biomol. Ther. (Seoul) 23 (4), 345-349. doi: 10.4062/ biomolther.2015.019

Hosseini, S. A., Zilaee, M., Shoushtari, M. H., and Ghasemi Dehcheshmeh, M. (2018). An evaluation of the effect of saffron supplementation on the antibody titer to heat-shock protein (HSP) 70, hsCRP and spirometry test in patients with mild and moderate persistent allergic asthma: A triple-blind, randomized placebo-controlled trial. Respir. Med. 145, 28-34. doi: 10.1016/ j.rmed.2018.10.016

Hu, Y., Wei, M., Niu, Q., Ma, R., Li, Y., Wang, X., et al. (2019). Grape seed proanthocyanidin extract alleviates arsenic-induced lung damage through NFkappaB signaling. Exp. Biol. Med. (Maywood) 244 (3), 213-226. doi: 10.1177/ 1535370219829881

Hussein, G., Miyashiro, H., Nakamura, N., Hattori, M., Kakiuchi, N., and Shimotohno, K. (2000). Inhibitory effects of sudanese medicinal plant extracts on hepatitis C virus (HCV) protease. Phytother. Res. 14 (7), 510516. doi: 10.1002/1099-1573(200011)14:7

Jin, Y. N., Lin, Z. J., Zhang, B., and Bai, Y. F. (2018). Effects of Chicory on Serum Uric Acid, Renal Function, and GLUT9 Expression in Hyperuricaemic Rats with Renal Injury and In Vitro Verification with Cells. Evid Based Complement. Alternat. Med. 2018, 1764212. doi: 10.1155/2018/1764212

Jung, Y. C., Kim, M. E., Yoon, J. H., Park, P. R., Youn, H. Y., Lee, H. W., et al. (2014). Anti-inflammatory effects of galangin on lipopolysaccharide-activated macrophages via ERK and NF-kappaB pathway regulation. Immunopharmacol. Immunotoxicol. 36 (6), 426-432. doi: 10.3109/ 08923973.2014.968257

Kandhare, A. D., Bodhankar, S. L., Mohan, V., and Thakurdesai, P. A. (2015). Effect of glycosides based standardized fenugreek seed extract in bleomycininduced pulmonary fibrosis in rats: Decisive role of Bax, Nrf2, NF-kappaB, Muc5ac, TNF-alpha and IL-1beta. Chem. Biol. Interact. 237, 151-165. doi: 10.1016/j.cbi.2015.06.019

Kapoor, M. P., Suzuki, K., Derek, T., Ozeki, M., and Okubo, T. (2020). Clinical evaluation of Emblica Officinalis Gatertn (Amla) in healthy human subjects: Health benefits and safety results from a randomized, double-blind, crossover placebo-controlled study. Contemp. Clin. Trials Commun. 17, 100499. doi: 10.1016/j.conctc.2019.100499

Kenari, H. M., Kordafshari, G., Kamalinejad, M., Aghighi, S., and Moghimi, M. (2018). Treatment of Hospital Acquired Pneumonia (HAP) with Persian medicine (PM): A Case Report. Tradit. Integr. Med. 3 (2), 69-77.

Kianbakht, S., and Ghazavi, A. (2011). Immunomodulatory effects of saffron: a randomized double-blind placebo-controlled clinical trial. Phytother. Res. 25 (12), 1801-1805. doi: 10.1002/ptr.3484

Kim, J. H., Mun, Y. J., Woo, W. H., Jeon, K. S., An, N. H., and Park, J. S. (2002). Effects of the ethanol extract of Cichorium intybus on the immunotoxicity by ethanol in mice. Int. Immunopharmacol. 2 (6), 733-744. doi: 10.1016/s15675769(02)00008-5

Kim, Y. M., Kim, H. J., and Chang, K. C. (2015). Glycyrrhizin reduces HMGB1 secretion in lipopolysaccharide-activated RAW 264.7 cells and endotoxemic mice by $\mathrm{p} 38 / \mathrm{Nrf2}$-dependent induction of HO-1. Int. Immunopharmacol. 26 (1), 112-118. doi: 10.1016/j.intimp.2015.03.014

Kim, S. J., Lee, J. W., Eun, Y. G., Lee, K. H., Yeo, S. G., and Kim, S. W. (2019). Pretreatment with a grape seed proanthocyanidin extract downregulates proinflammatory cytokine expression in airway epithelial cells infected with respiratory syncytial virus. Mol. Med. Rep. 19 (4), 3330-3336. doi: 10.3892/ mmr.2019.9967

Konno, K., Sawamura, R., Sun, Y., Yasukawa, K., Shimizu, T., Watanabe, W., et al. (2011). Antiviral activities of diarylheptanoids isolated from Alpinia officinarum against respiratory syncytial virus, poliovirus, measles virus, and herpes simplex virus type 1 in vitro. Nat. Prod. Commun. 6 (12), 1881-1884. doi: $10.1177 / 1934578$ X1100601222

Kopaei, R., Khajegir, A., and Kiani, S. (2016). The Association between dystemperament and prevention of diseases: A systematic review. J. Clin. Diagn. Res. 10 (9), YE01-YE06. doi: 10.7860/JCDR/2016/19023.8511

Lee, H. H., Shin, J. S., Lee, W. S., Ryu, B., Jang, D. S., and Lee, K. T. (2016). Biflorin, Isolated from the Flower Buds of Syzygium aromaticum L., Suppresses LPSInduced Inflammatory Mediators via STAT1 Inactivation in Macrophages and Protects Mice from Endotoxin Shock. J. Nat. Prod. 79 (4), 711-720. doi: 10.1021/acs.jnatprod.5b00609

Lee, J. W., Kim, Y. I., Im, C. N., Kim, S. W., Kim, S. J., Min, S., et al. (2017). Grape Seed Proanthocyanidin Inhibits Mucin Synthesis and Viral Replication by Suppression of AP-1 and NF-kappaB via p38 MAPKs/JNK Signaling Pathways in Respiratory Syncytial Virus-Infected A549 Cells. J. Agric. Food Chem. 65 (22), 4472-4483. doi: 10.1021/acs.jafc.7b00923

Lepczynski, A., Herosimczyk, A., Ozgo, M., Skomial, J., Taciak, M., Barszcz, M., et al. (2015). Dietary supplementation with dried chicory root triggers changes in the blood serum proteins engaged in the clotting process and the innate immune response in growing pigs. J. Physiol. Pharmacol. 66 (1), 47-55.

Li, L., Sun, T., Tian, J., Yang, K., Yi, K., and Zhang, P. (2013). Garlic in clinical practice: an evidence-based overview. Crit. Rev. Food Sci. Nutr. 53 (7), 670-681. doi: 10.1080/10408398.2010.537000

Li, M., Yan, Y. X., Yu, Q. T., Deng, Y., Wu, D. T., Wang, Y., et al. (2017). Comparison of Immunomodulatory Effects of Fresh Garlic and Black Garlic Polysaccharides on RAW 264.7 Macrophages. J. Food Sci. 82 (3), 765-771. doi: 10.1111/1750-3841.13589

Li, M., Wen, Z., Xue, Y., Han, X., Ma, D., Ma, Z., et al. (2019). Cardioprotective effects of glycyrrhizic acid involve inhibition of calcium influx via L-type 
calcium channels and myocardial contraction in rats. Naunyn Schmiedebergs Arch. Pharmacol. 393, 979-989. doi: 10.1007/s00210-019-01767-3

Lin, L.-T., Hsu, W.-C., and Lin, C.-C. (2014). Antiviral natural products and herbal medicines. J. Tradit. Complement. Med. 4 (1), 24-35. doi: 10.4103/22254110.124335

Liu, Q., Jiang, J. X., Liu, Y. N., Ge, L. T., Guan, Y., Zhao, W., et al. (2017). Grape seed extract ameliorates bleomycin-induced mouse pulmonary fibrosis. Toxicol. Lett. 273, 1-9. doi: 10.1016/j.toxlet.2017.03.012

Liu, J., Li, Y., Tang, Y., Cheng, J., Wang, J., Li, J., et al. (2018). Rhein protects the myocardiac cells against hypoxia/reoxygention-induced injury by suppressing GSK3beta activity. Phytomedicine 51, 1-6. doi: 10.1016/j.phymed.2018.06.029

Long, M., Zhang, Y., Li, P., Yang, S. H., Zhang, W. K., Han, J. X., et al. (2016). Intervention of Grape Seed Proanthocyanidin Extract on the Subchronic Immune Injury in Mice Induced by Aflatoxin B1. Int. J. Mol. Sci. 17 (4), 516. doi: 10.3390/ijms17040516

Lu, J., Jiang, H., Liu, B., Baiyun, R., Li, S., Lv, Y., et al. (2018). Grape seed procyanidin extract protects against $\mathrm{Pb}$-induced lung toxicity by activating the AMPK/Nrf2/p62 signaling axis. Food Chem. Toxicol. 116 (Pt B), 59-69. doi: $10.1016 /$ j.fct.2018.03.034

Lu, J., Li, J., Hu, Y., Guo, Z., Sun, D., Wang, P., et al. (2019). Chrysophanol protects against doxorubicin-induced cardiotoxicity by suppressing cellular PARylation. Acta Pharm. Sin. $B 9$ (4), 782-793. doi: 10.1016/j.apsb.2018.10.008

Ma, C., Ma, Z., Liao, X. L., Liu, J., Fu, Q., and Ma, S. (2013). Immunoregulatory effects of glycyrrhizic acid exerts anti-asthmatic effects via modulation of Th1/ Th2 cytokines and enhancement of CD4(+)CD25(+)Foxp3+ regulatory T cells in ovalbumin-sensitized mice. J. Ethnopharmacol. 148 (3), 755-762. doi: $10.1016 /$ j.jep.2013.04.021

Magalhaes, C. B., Riva, D. R., DePaula, L. J., Brando-Lima, A., Koatz, V. L., LealCardoso, J. H., et al. (2010). In vivo anti-inflammatory action of eugenol on lipopolysaccharide-induced lung injury. J. Appl. Physiol. (1985) 108 (4), 845851. doi: 10.1152/japplphysiol.00560.2009

Mahmood, N., Piacente, S., Pizza, C., Burke, A., Khan, A. I., and Hay, A. J. (1996). The anti-HIV activity and mechanisms of action of pure compounds isolated from Rosa damascena. Biochem. Biophys. Res. Commun. 229 (1), 73-79. doi: 10.1006/bbrc.1996.1759

Malik, S., Suchal, K., Bhatia, J., Khan, S. I., Vasisth, S., Tomar, A., et al. (2016). Therapeutic Potential and Molecular Mechanisms of Emblica officinalis Gaertn in Countering Nephrotoxicity in Rats Induced by the Chemotherapeutic Agent Cisplatin. Front. Pharmacol. 7, 350. doi: 10.3389/fphar.2016.00350

McGrath, J. C., and Lilley, E. (2015). Implementing guidelines on reporting research using animals (ARRIVE etc.): new requirements for publication in BJP. Br. J. Pharmacol. 172 (13), 3189-3193. doi: 10.1111/bph.13112/abstract

Mehdizadeh, R., Parizadeh, M. R., Khooei, A. R., Mehri, S., and Hosseinzadeh, H. (2013). Cardioprotective effect of saffron extract and safranal in isoproterenolinduced myocardial infarction in wistar rats. Iran J. Basic Med. Sci. 16 (1), 56-63. doi: $10.22038 / \mathrm{ijbms} .2013 .249$

Mehta, P., McAuley, D. F., Brown, M., Sanchez, E., Tattersall, R. S., and Manson, J. J. (2020). COVID-19: consider cytokine storm syndromes and immunosuppression. Lancet 395 (10229), 1033-1034. doi: 10.1016/S0140-6736(20)30628-0

Michaelis, M., Geiler, J., Naczk, P., Sithisarn, P., Leutz, A., Doerr, H. W., et al. (2011). Glycyrrhizin exerts antioxidative effects in H5N1 influenza A virusinfected cells and inhibits virus replication and pro-inflammatory gene expression. PloS One 6 (5), e19705. doi: 10.1371/journal.pone.0019705

Miltonprabu, S., Sumedha, N. C., and Senthilraja, P. (2017). Diallyl trisulfide, a garlic polysulfide protects against As-induced renal oxidative nephrotoxicity, apoptosis and inflammation in rats by activating the Nrf2/ARE signaling pathway. Int. Immunopharmacol. 50, 107-120. doi: 10.1016/j.intimp.2017.06.011

Mo, M., Li, S., Dong, Z., Li, C., Sun, Y., Li, A., et al. (2020). S-allylmercaptocysteine ameliorates lipopolysaccharide-induced acute lung injury in mice by inhibiting inflammation and oxidative stress via nuclear factor kappa B and Keap1/Nrf2 pathways. Int. Immunopharmacol. 81, 106273. doi: 10.1016/j.intimp.20a20.106273

Mohi El-Din, M. M., Mostafa, A. M., and Abd-Elkader, A. (2014). Experimental studies on the effect of (Lambda-Cyhalothrin) insecticide on lungs and the ameliorating effect of plant extracts (Ginseng (Panax Ginseng) and garlic (Allium sativum L.) on asthma development in albino rats. BMC Res. Notes 7 , 243. doi: 10.1186/1756-0500-7-243

Mukthamba, P., and Srinivasan, K. (2015). Hypolipidemic influence of dietary fenugreek (Trigonella foenum-graecum) seeds and garlic (Allium sativum) in experimental myocardial infarction. Food Funct. 6 (9), 3117-3125. doi: $10.1039 / \mathrm{c} 5$ fo00240k

Nantz, M. P., Rowe, C. A., Muller, C. E., Creasy, R. A., Stanilka, J. M., and Percival, S. S. (2012). Supplementation with aged garlic extract improves both NK and gammadelta-T cell function and reduces the severity of cold and flu symptoms: a randomized, double-blind, placebo-controlled nutrition intervention. Clin. Nutr. 31 (3), 337-344. doi: 10.1016/j.clnu.2011.11.019

Nassan, M. A., Mohamed, E. H., Abdelhafez, S., and Ismail, T. A. (2015). Effect of clove and cinnamon extracts on experimental model of acute hematogenous pyelonephritis in albino rats: Immunopathological and antimicrobial study. Int. J. Immunopathol. Pharmacol. 28 (1), 60-68. doi: 10.1177/ 0394632015572075

Nassiri-Asl, M., and Hosseinzadeh, H. (2016). Review of the pharmacological effects of Vitis vinifera (Grape) and its bioactive constituents: an update. Phytother. Res. 30 (9), 1392-1403. doi: 10.1002/ptr.5644

Nayebi, N., Khalili, N., Kamalinejad, M., and Emtiazy, M. (2017). A systematic review of the efficacy and safety of Rosa damascena Mill. with an overview on its phytopharmacological properties. Complement. Ther. Med. 34, 129-140. doi: 10.1016/j.ctim.2017.08.014

Nicolis, E., Lampronti, I., Dechecchi, M. C., Borgatti, M., Tamanini, A., Bianchi, N., et al. (2008). Pyrogallol, an active compound from the medicinal plant Emblica officinalis, regulates expression of pro-inflammatory genes in bronchial epithelial cells. Int. Immunopharmacol. 8 (12), 1672-1680. doi: 10.1016/j.intimp.2008.08.001

Nie, Y., Yu, K., Li, B., Hu, Y., Zhang, H., Xin, R., et al. (2019). S-allyl-1-cysteine attenuates bleomycin-induced pulmonary fibrosis and inflammation via AKT/ NF-kappaB signaling pathway in mice. J. Pharmacol. Sci. 139 (4), 377-384. doi: 10.1016/j.jphs.2019.03.002

Nishimoto, Y., Hisatsune, A., Katsuki, H., Miyata, T., Yokomizo, K., and Isohama, Y. (2010). Glycyrrhizin attenuates mucus production by inhibition of MUC5AC mRNA expression in vivo and in vitro. J. Pharmacol. Sci. 113 (1), 76-83. doi: 10.1254/jphs.09344fp

Ojha, S., Golechha, M., Kumari, S., and Arya, D. S. (2012). Protective effect of Emblica officinalis (amla) on isoproterenol-induced cardiotoxicity in rats. Toxicol. Ind. Health 28 (5), 399-411. doi: 10.1177/0748233711413798

Ojha, S., Golechha, M., Kumari, S., Bhatia, J., and Arya, D. S. (2013). Glycyrrhiza glabra protects from myocardial ischemia-reperfusion injury by improving hemodynamic, biochemical, histopathological and ventricular function. Exp. Toxicol. Pathol. 65 (1-2), 219-227. doi: 10.1016/j.etp.2011.08.011

Padiya, R., Chowdhury, D., Borkar, R., Srinivas, R., Pal Bhadra, M., and Banerjee, S. K. (2014). Garlic attenuates cardiac oxidative stress via activation of PI3K/ AKT/Nrf2-Keap1 pathway in fructose-fed diabetic rat. PloS One 9 (5), e94228. doi: 10.1371/journal.pone.0094228

Panda, S., Biswas, S., and Kar, A. (2013). Trigonelline isolated from fenugreek seed protects against isoproterenol-induced myocardial injury through downregulation of Hsp27 and alphaB-crystallin. Nutrition 29 (11-12), 1395-1403. doi: 10.1016/j.nut.2013.05.006

Panda, S., and Kar, A. (2010). A novel phytochemical, digoxigenin-3-O-rutin in the amelioration of isoproterenol-induced myocardial infarction in rat: a comparison with digoxin. Cardiovasc. Ther. 30 (3), 125-135. doi: 10.1111/ j.1755-5922.2010.00242.x

Pastorino, G., Cornara, L., Soares, S., Rodrigues, F., and Oliveira, M. B. P. (2018). Liquorice (Glycyrrhiza glabra): A phytochemical and pharmacological review. Phytother. Res. 32 (12), 2323-2339. doi: 10.1002/ptr.6178

Patel, S. S., and Goyal, R. K. (2011). Prevention of diabetes-induced myocardial dysfunction in rats using the juice of the Emblica officinalis fruit. Exp. Clin. Cardiol. 16 (3), 87-91. doi: 10.4103/0974-8490.89743

Patra, J. K., Das, G., Bose, S., Banerjee, S., Vishnuprasad, C. N., del Pilar Rodriguez-Torres, M., et al. (2020). Star anise (Illicium verum): Chemical compounds, antiviral properties, and clinical relevance. Phytother. Res. 34 (6), 1248-1267. doi: 10.1002/ptr.6614

Pingali Usharani, N. F., and Muralidhar, N. (2013). Effects of Phyllanthus emblica extract on endothelial dysfunction and biomarkers of oxidative stress in patients with type 2 diabetes mellitus: a randomized, double-blind, controlled study. Diabetes Metab. Syndr. Obes. 6, 275-284. doi: 10.2147/ DMSO.S46341

Pourfarjam, Y., Rezagholizadeh, L., Nowrouzi, A., Meysamie, A., Ghaseminejad, S., Ziamajidi, N., et al. (2017). Effect of Cichorium intybus L. seed extract on renal 
parameters in experimentally induced early and late diabetes type 2 in rats. Ren. Fail 39 (1), 211-221. doi: 10.1080/0886022x.2016.1256317

Pradeep, S. R., Barman, S., and Srinivasan, K. (2019). Attenuation of diabetic nephropathy by dietary fenugreek (Trigonella foenum-graecum) seeds and onion (Allium cepa) via suppression of glucose transporters and reninangiotensin system. Nutrition 67-68, 110543. doi: 10.1016/j.nut.2019.06.024

Pradeep, S. R., and Srinivasan, K. (2018). Alleviation of Cardiac Damage by Dietary Fenugreek (Trigonella foenum-graecum) Seeds is Potentiated by Onion (Allium cepa) in Experimental Diabetic Rats via Blocking ReninAngiotensin System. Cardiovasc. Toxicol. 18 (3), 221-231. doi: 10.1007/ s12012-017-9431-1

Pretorius, R., Prescott, S. L., and Palmer, D. J. (2018). Taking a prebiotic approach to early immunomodulation for allergy prevention. Expert Rev. Clin. Immunol. 14 (1), 43-51. doi: 10.1080/1744666X.2018.1411191

Qi, W., Boliang, W., Xiaoxi, T., Guoqiang, F., Jianbo, X., and Gang, W. (2020). Cardamonin protects against doxorubicin-induced cardiotoxicity in mice by restraining oxidative stress and inflammation associated with Nrf2 signaling. BioMed. Pharmacother. 122, 109547. doi: 10.1016/j.biopha.2019.109547

Qu, L., Chen, C., He, W., Chen, Y., Li, Y., Wen, Y., et al. (2019). Glycyrrhizic acid ameliorates LPS-induced acute lung injury by regulating autophagy through the PI3K/AKT/mTOR pathway. Am. J. Transl. Res. 11 (4), 2042-2055.

Rahim, V. B., Khammar, M. T., Rakhshandeh, H., Samzadeh-Kermani, A., Hosseini, A., and Askari, V. R. (2019). Crocin protects cardiomyocytes against LPS-Induced inflammation. Pharmacol. Rep. 71 (6), 1228-1234. doi: 10.1016/j.pharep.2019.07.007

Ramadan, G., El-Beih, N. M., and Abd El-Kareem, H. F. (2011). Anti-metabolic syndrome and immunostimulant activities of Egyptian fenugreek seeds in diabetic/obese and immunosuppressive rat models. Br. J. Nutr. 105 (7), 9951004. doi: $10.1017 / \mathrm{s} 0007114510004708$

Rhazes, M. I. Z. (960). The great continens (Alhavi-fil-Tib).Transaleted by afsharipour. S Translation]).

Razmaraii, N., Babaei, H., Mohajjel Nayebi, A., Assadnassab, G., Ashrafi Helan, J., and Azarmi, Y. (2016). Crocin treatment prevents doxorubicin-induced cardiotoxicity in rats. Life Sci. 157, 145-151. doi: 10.1016/j.lfs.2016.06.012

Rezaee-Khorasany, A., Razavi, B. M., Taghiabadi, E., Tabatabaei Yazdi, A., and Hosseinzadeh, H. (2019). Effect of saffron (stigma of Crocus sativus L.) aqueous extract on ethanol toxicity in rats: A biochemical, histopathological and molecular study. J. Ethnopharmacol. 237, 286-299. doi: 10.1016/ j.jep.2019.03.048

Ruetzler, K., Fleck, M., Nabecker, S., Pinter, K., Landskron, G., Lassnigg, A., et al. (2013). A randomized, double-blind comparison of licorice versus sugar-water gargle for prevention of postoperative sore throat and postextubation coughing. Anesth. Analg. 117 (3), 614-621. doi: 10.1213/ANE.0b013e318299a650

Sachdeva, J., Tanwar, V., Golechha, M., Siddiqui, K. M., Nag, T. C., Ray, R., et al. (2012). Crocus sativus L. (saffron) attenuates isoproterenol-induced myocardial injury via preserving cardiac functions and strengthening antioxidant defense system. Exp. Toxicol. Pathol. 64 (6), 557-564. doi: 10.1016/j.etp.2010.11.013

Sadati, S. N., Ardekani, M. R. S., Ebadi, N., Yakhchali, M., Dana, A. R., Masoomi, F., et al. (2016). Review of scientific evidence of medicinal convoy plants in traditional Persian medicine. Pharmacogn. Rev. 10 (19), 33. doi: 10.4103/09737847.176546

Sai Ram, M., Neetu, D., Yogesh, B., Anju, B., Dipti, P., Pauline, T., et al. (2002). Cyto-protective and immunomodulating properties of Amla (Emblica officinalis) on lymphocytes: an in-vitro study. J. Ethnopharmacol. 81 (1), 510. doi: 10.1016/s0378-8741(01)00421-4

Sai Ram, M., Neetu, D., Deepti, P., Vandana, M., Ilavazhagan, G., Kumar, D., et al. (2003). Cytoprotective activity of Amla (Emblica officinalis) against chromium (VI) induced oxidative injury in murine macrophages. Phytother. Res. 17 (4), 430-433. doi: 10.1002/ptr.1157

Sawamura, R., Shimizu, T., Sun, Y., Yasukawa, K., Miura, M., Toriyama, M., et al. (2010). In vitro and in vivo anti-influenza virus activity of diarylheptanoids isolated from Alpinia officinarum. Antivir. Chem. Chemother. 21 (1), 33-41. doi: 10.3851/imp1676

Schepetkin, I. A., Kirpotina, L. N., Khlebnikov, A. I., Balasubramanian, N., and Quinn, M. T. (2019). Neutrophil Immunomodulatory Activity of Natural Organosulfur Compounds. Molecules 24 (9), 1809. doi: 10.3390/ molecules24091809
Seo, J. W., Cho, S. C., Park, S. J., Lee, E. J., Lee, J. H., Han, S. S., et al. (2013). 1'Acetoxychavicol acetate isolated from Alpinia galanga ameliorates ovalbumininduced asthma in mice. PloS One 8 (2), e56447. doi: 10.1371/ journal.pone.0056447

Shahrajabian, M. H., Khoshkharam, M., Zandi, P., Sun, W., and Cheng, Q. (2019). Jujube, a super-fruit in traditional Chinese medicine, heading for modern pharmacological science. J. Med. Plant Stud. 7 (4), 173-178.

Shen, C., Zhang, Z., Xie, T., Ji, J., Xu, J., Lin, L., et al. (2019). Rhein Suppresses Lung Inflammatory Injury Induced by Human Respiratory Syncytial Virus Through Inhibiting NLRP3 Inflammasome Activation via NF-kappaB Pathway in Mice. Front. Pharmacol. 10, 1-11. doi: 10.3389/fphar.2019.01600

Shu, Y. S., Tao, W., Miao, Q. B., Lu, S. C., and Zhu, Y. B. (2014). Galangin dampens mice lipopolysaccharide-induced acute lung injury. Inflammation 37 (5), 1661-1668. doi: 10.1007/s10753-014-9894-1

Siahpoosh, M. B. (2020). How Can Persian Medicine (Traditional Iranian Medicine) Be Effective to Control COVID-19? Tradit. Integr. Med. 5 (2), 46-48.

Sivakumar, A. S., Viswanathan, P., and Anuradha, C. V. (2010). Dose-dependent effect of galangin on fructose-mediated insulin resistance and oxidative events in rat kidney. Redox Rep. 15 (5), 224-232. doi: 10.1179/135100010x12826446921545

Soleymani, S., Bahramsoltani, R., Rahimi, R., and Abdollahi, M. (2017). Clinical risks of St John's Wort (Hypericum perforatum) co-administration. Expert Opin. Drug Metab. Toxicol. 13 (10), 1047-1062. doi: 10.1080/ 17425255.2017.1378342

Soleymani, S., Zabihollahi, R., Shahbazi, S., and Bolhassani, A. (2018). Antiviral Effects of Saffron and its Major Ingredients. Curr. Drug Deliv. 15 (5), 698-704. doi: 10.2174/1567201814666171129210654

Song, G., Zhang, Y., Yu, S., Lv, W., Guan, Z., Sun, M., et al. (2019). Chrysophanol attenuates airway inflammation and remodeling through nuclear factor-kappa B signaling pathway in asthma. Phytother. Res. 33 (10), 2702-2713. doi: $10.1002 /$ ptr.6444

Street, R. A., Sidana, J., and Prinsloo, G. (2013). Cichorium intybus: Traditional uses, phytochemistry, pharmacology, and toxicology. Evid Based Complement. Alternat. Med. 2013, 579319. doi: 10.1155/2013/579319

Su, H., Yang, M., Wan, C., Yi, L. X., Tang, F., Zhu, H. Y., et al. (2020). Renal histopathological analysis of 26 postmortem findings of patients with COVID19 in China. Kidney Int. 98 (1), 219-227. doi: 10.1016/j.kint.2020.04.003

Tamura, S., Shiomi, A., Kaneko, M., Ye, Y., Yoshida, M., Yoshikawa, M., et al. (2009). New Rev-export inhibitor from Alpinia galanga and structure-activity relationship. Bioorg. Med. Chem. Lett. 19 (9), 2555-2557. doi: 10.1016/ j.bmcl.2009.03.047

Thirunavukkarasu, M., Selvaraju, V., Tapias, L., Sanchez, J. A., Palesty, J. A., and Maulik, N. (2015). Protective effects of Phyllanthus emblica against myocardial ischemia-reperfusion injury: the role of PI3-kinase/glycogen synthase kinase 3beta/beta-catenin pathway. J. Physiol. Biochem. 71 (4), 623-633. doi: 10.1007/ s13105-015-0426-8

Thuy, B. T. P., My, T. T. A., Hai, N. T. T., Hieu, L. T., Hoa, T. T., Thi Phuong Loan, $\mathrm{H}$., et al. (2020). Investigation into SARS-CoV-2 resistance of compounds in garlic essential oil. ACS Omega 5 (14), 8312-8320. doi: 10.1021/ acsomega.0c00772

Tripathi, U. N., and Chandra, D. (2009). The plant extracts of Momordica charantia and Trigonella foenum-graecum have anti-oxidant and antihyperglycemic properties for cardiac tissue during diabetes mellitus. Oxid. Med. Cell. Longev. 2 (5), 290-296. doi: 10.4161/oxim.2.5.9529

Tu, Y., Gu, L., Chen, D., Wu, W., Liu, H., Hu, H., et al. (2017). Rhein Inhibits Autophagy in Rat Renal Tubular Cells by Regulation of AMPK/mTOR Signaling. Sci. Rep. 7, 43790. doi: 10.1038/srep43790

Ulusoy, S., Ozkan, G., Mungan, S., Orem, A., Yulug, E., Alkanat, M., et al. (2014). GSPE is superior to NAC in the prevention of contrast-induced nephropathy: might this superiority be related to caspase 1 and calpain 1? Life Sci. 103 (2), 101-110. doi: 10.1016/j.lfs.2014.03.030

Upadhyay, S., Mantha, A. K., and Dhiman, M. (2020). Glycyrrhiza glabra (Licorice) root extract attenuates doxorubicin-induced cardiotoxicity via alleviating oxidative stress and stabilising the cardiac health in $\mathrm{H} 9 \mathrm{c} 2$ cardiomyocytes. J. Ethnopharmacol. 258, 112690. doi: 10.1016/ j.jep. 2020.112690

Upadya, H., Prabhu, S., Prasad, A., Subramanian, D., Gupta, S., and Goel, A. (2019). A randomized, double blind, placebo controlled, multicenter clinical trial to assess the efficacy and safety of Emblica officinalis extract in patients 
with dyslipidemia. BMC Complement. Altern. Med. 19 (1), 27. doi: 10.1186/ s12906-019-2430-y

Wang, L., Yang, R., Yuan, B., Liu, Y., and Liu, C. (2015). The antiviral and antimicrobial activities of licorice, a widely-used Chinese herb. Acta Pharm. Sin. B 5 (4), 310-315. doi: 10.1016/j.apsb.2015.05.005

Wang, C., Li, J., Song, G. L., Niu, Q., Xu, S. Z., Feng, G. L., et al. (2017). Grape Seed Procyanidin Extract Reduces Arsenic-Induced Renal Inflammatory Injury in Male Mice. BioMed. Environ. Sci. 30 (7), 535-539. doi: 10.3967/bes2017.071

Wang, X., Jia, D., Zhang, J., and Wang, W. (2017). Grape seed proanthocyanidins protect cardiomyocytes against hypoxia/reoxygenation injury by attenuating endoplasmic reticulum stress through PERK/eIF2alpha pathway. Mol. Med. Rep. 16 (6), 9189-9196. doi: 10.3892/mmr.2017.7756

Watanabe, K., Takatsuki, H., Sonoda, M., Tamura, S., Murakami, N., and Kobayashi, N. (2011). Anti-influenza viral effects of novel nuclear export inhibitors from Valerianae Radix and Alpinia galanga. Drug Discov. Ther. 5 (1), 26-31. doi: 10.5582/ddt.v5.1.26

World Health Organization. (2013). WHO traditional medicine strategy: 20142023. World health Organization. Available online at: www.who.int.

World Health Organization. (2020). Coronavirus disease 2019 (COVID-19): situation report, 197. World health Organization. Available online at: www. who.int.

Wu, J. T., Leung, K., Bushman, M., Kishore, N., Niehus, R., de Salazar, P. M., et al. (2020). Estimating clinical severity of COVID-19 from the transmission dynamics in Wuhan, China. Nat. Med. 26 (4), 506-510. doi: 10.1038/ s41591-020-0822-7

Xiong, H. R., Shen, Y. Y., Lu, L., Hou, W., Luo, F., Xiao, H., et al. (2012). The inhibitory effect of Rheum palmatum against coxsackievirus B3 in vitro and in vivo. Am. J. Chin. Med. 40 (4), 801-812. doi: 10.1142/s0192415x12500607

Xiu-hui, L., Ke, Z., Jian-hua, H., Xin-hui, G., Zhong-jie, H., Yu, Y., et al. (2003). Clinical evaluation of integrative Chinese and western medicine in treating SARS. Chin. J. Integr. Med. 9 (3), 181-184. doi: 10.1007/BF02838028

Yadav, S. S., Singh, M. K., Singh, P. K., and Kumar, V. (2017). Traditional knowledge to clinical trials: A review on therapeutic actions of Emblica officinalis. BioMed. Pharmacother. 93, 1292-1302. doi: 10.1016/ j.biopha.2017.07.065

Yang, Y., Islam, M. S., Wang, J., Li, Y., and Chen, X. (2020). Traditional Chinese medicine in the treatment of patients infected with 2019-new coronavirus (SARS-CoV-2): a review and perspective. Int. J. Biol. Sci. 16 (10), 1708. doi: $10.7150 /$ ijbs. 45538

Yu, L., Di, W., Dong, X., Li, Z., Xue, X., Zhang, J., et al. (2017). Diallyl trisulfide exerts cardioprotection against myocardial ischemia-reperfusion injury in diabetic state, role of AMPK-mediated AKT/GSK-3beta/HIF-1alpha activation. Oncotarget 8 (43), 74791-74805. doi: 10.18632/oncotarget.20422

Zaghloul, M. S., Said, E., Suddek, G. M., and Salem, H. A. (2019). Crocin attenuates lung inflammation and pulmonary vascular dysfunction in a rat model of bleomycin-induced pulmonary fibrosis. Life Sci. 235, 116794. doi: 10.1016/ j.lfs.2019.116794

Zha, W. J., Qian, Y., Shen, Y., Du, Q., Chen, F. F., Wu, Z. Z., et al. (2013). Galangin Abrogates Ovalbumin-Induced Airway Inflammation via Negative Regulation of NF-kappaB. Evid Based Complement. Alternat. Med. 2013, 767689. doi: $10.1155 / 2013 / 767689$
Zhang, Z. H., Li, M. H., Liu, D., Chen, H., Chen, D. Q., Tan, N. H., et al. (2018) Rhubarb Protect Against Tubulointerstitial Fibrosis by Inhibiting TGF-beta/ Smad Pathway and Improving Abnormal Metabolome in Chronic Kidney Disease. Front. Pharmacol. 9, 1029. doi: 10.3389/fphar.2018.01029

Zhang, D. H., Wu, K. L., Zhang, X., Deng, S. Q., and Peng, B. (2020a). In silico screening of Chinese herbal medicines with the potential to directly inhibit 2019 novel coronavirus. J. Integr. Med. 18 (2), 152-158. doi: 10.1016/ j.joim.2020.02.005

Zhang, D., Qi, B. Y., Zhu, W. W., Huang, X., and Wang, X. Z. (2020b). Crocin alleviates lipopolysaccharide-induced acute respiratory distress syndrome by protecting against glycocalyx damage and suppressing inflammatory signaling pathways. Inflammation Res. 69 (3), 267-278. doi: 10.1007/s00011-019-01314-Z

Zhao, H., Zhao, M., Wang, Y., Li, F., and Zhang, Z. (2016). Glycyrrhizic Acid Attenuates Sepsis-Induced Acute Kidney Injury by Inhibiting NF-kappaB Signaling Pathway. Evid Based Complement. Alternat. Med. 2016, 8219287. doi: $10.1155 / 2016 / 8219287$

Zhao, R., Xie, E., Yang, X., and Gong, B. (2019). Alliin alleviates myocardial ischemia-reperfusion injury by promoting autophagy. Biochem. Biophys. Res. Commun. 512 (2), 236-243. doi: 10.1016/j.bbrc.2019.03.046

Zheng, Q. X., Wu, H. F., Jian, G., Nan, H. J., Chen, S. L., Yang, J. S., et al. (2013). Review of rhubarbs: chemistry and pharmacology. Chin. Herb. Med. 5 (1), 9-32. doi: 10.7501/j.issn.1674-6384.2013.01.003

Zheng, Y. Y., Ma, Y. T., Zhang, J. Y., and Xie, X. (2020). COVID-19 and the cardiovascular system. Nat. Rev. Cardiol. 17 (5), 259-260. doi: 10.1038/s41569020-0360-5

Zhong, T., Zhang, L. Y., Wang, Z. Y., Wang, Y., Song, F. M., Zhang, Y. H., et al. (2017). Rheum emodin inhibits enterovirus 71 viral replication and affects the host cell cycle environment. Acta Pharmacol. Sin. 38 (3), 392-401. doi: 10.1038/aps.2016.110

Zhou, G. H., Zhang, F., Wang, X. N., Kwon, O. J., Kang, D. G., Lee, H. S., et al. (2014). Emodin accentuates atrial natriuretic peptide secretion in cardiac atria. Eur. J. Pharmacol. 735, 44-51. doi: 10.1016/j.ejphar.2014.04.014

Zhu, X., Jiang, X., Li, A., Zhao, Z., and Li, S. (2017). S-Allylmercaptocysteine Attenuates Cisplatin-Induced Nephrotoxicity through Suppression of Apoptosis, Oxidative Stress, and Inflammation. Nutrients 9 (2), 166. doi: 10.3390/nu9020166

Zou, M., Chen, Y., Sun-Waterhouse, D., Zhang, Y., and Li, F. (2018). Immunomodulatory acidic polysaccharides from Zizyphus jujuba cv. Huizao: Insights into their chemical characteristics and modes of action. Food Chem. 258, 35-42. doi: 10.1016/j.foodchem.2018.03.052

Conflict of Interest: The authors declare that the research was conducted in the absence of any commercial or financial relationships that could be construed as a potential conflict of interest.

Copyright (c) 2020 Bahramsoltani and Rahimi. This is an open-access article distributed under the terms of the Creative Commons Attribution License (CC BY). The use, distribution or reproduction in other forums is permitted, provided the original author(s) and the copyright owner(s) are credited and that the original publication in this journal is cited, in accordance with accepted academic practice. No use, distribution or reproduction is permitted which does not comply with these terms. 